\title{
Quantum Field Theories in All Dimensions
}

\author{
C.J. Read \\ Trinity College, Cambridge, CB2 1TQ, England (email cr25@cus.cam.ac.uk)
}

Received: 9 November 1994 / Accepted: 4 August 1995

\begin{abstract}
In this paper we exhibit a large class of hermitian scalar field theories satisfying the Wightman axioms. For each $d>0$, and each polynomial $P$, we exhibit a collection of theories which are loosely but legitimately based on a $P(\phi)$ interaction in $d$ space dimensions. One of the features of the construction is that the Wightman $n$-point function of each theory is a sum of finitely many integrals associated with "Feynman-like" graphs. Thus, it is in closed form.
\end{abstract}

\section{Introduction}

We present here a new approach to the construction of Wightman field theories. It needs a new idea to obtain nontrivial scattering, but there are several new elements in the proof of the Wightman axioms, of which we hope some may be useful in the construction of nontrivial theories.

Our theories are scalar field theories, based loosely but (we feel) genuinely on an arbitrary polynomial interaction in an arbitrary dimension. These theories satisfy the Wightman axioms, including vacuum uniqueness, and they are in some sense "perturbations" of a generalised free theory (see Definition 1.3).

However, they cannot be used directly to construct theories with nontrivial scattering amplitudes, firstly because the Wightman functions as given are "too smooth," a fact which cannot be remedied by merely adjusting things for fixed instead of "smoothly smeared" mass. Secondly, and more deeply, K.H.Rehren [Reh1] has succeeded in "decomposing" these theories into infinite Wick polnomials of countably many Gaussian fields with various smeared mass density functions (here an "infinite" polynomial means a polynomial in countably many variables with infinitely many nonzero coefficients but finite total degree, the coefficients being so chosen that the correlation functions converge in an appropriate manner). For the details of the "Rehren decomposition," one should of course consult [Reh1]; let us just remark that the decomposition is interesting in its own right, and by no means trivial. This result thus places our theories in the Borchers class of something we know to be trivial, and thus definitely precludes nontrivial scattering, at any rate in the case of fixed mass. 
Thus, our Wightman functions are obviously non-Gaussian, and with a little effort one can see that they are not Wick polynomials in a single Gaussian theory either. That they can, however, be decomposed into infinite Wick polynomials in countably many Gaussian fields, is the point of Rehren's proof.

However, there are some new ideas in this construction which we hope will prove useful.

The theories all involve summing certain integrals, indexed by certain graphs, in order to obtain the Wightman functions of the theory. One of the main features of the theory is that the number of graphs involved in calculating the Wightman $n$-point function is finite; there is an integer parameter $S$ of the theories such that the graphs involved with the $n$-point function have at most $n S$ internal vertices. That (with this choice of integrand) one can get a local and covariant scalar field theory with only finitely many graphs, is the main point of the proof.

We conjecture that the collection of graphs used might in some sense be "minimal" for a collection of graphs having all the useful properties that these ones have; for the details of our collection see $\S 2$.

In order to get our theories to work, we introduce various choices, notably an integer parameter $S$ which regulates the number of graphs that we use, and a smoothing function $\theta$ which acts a bit like a momentum cutoff.

This kind of cutoff does not, however, have to be removed in order to get the final answer; instead, covariance is restored by a judicious integration over several copies of the (full) Lorentz group $L$. Details of the integration are given in our definitions in $\S 3$.

The substitution required to establish covariance under a Lorentz transform $\gamma$ is that one takes all Lorentz group dummy variables $g_{e}$ involved in the integrations over $L$, and replaces them with elements $g_{e}\left(\gamma^{*}\right)^{-1}$, where $*$ denotes the transpose of the linear map (for details, see $\S 7$ ). Our integral remains invariant under the substitution because Haar measure is used for the integrations.

Of course the presence of integrations over the noncompact group $L$ raises questions of convergence; these are answered in $\S 4$. Positivity is shown by explicitly embedding the construction inside a suitably large Fock space $e^{K}$, where $K=L_{2}\left(\mathbb{R}^{d+1} \times L\right)$; this is done in $\S 5$.

Locality, also, is restored by a rather more complicated substitution involving our integrations over the Lorentz group. Each graph which we associate with the Wightman $n$-point function splits itself up naturally into $n$ "bands," one for each test function; and the group elements $g_{e}$ are indexed by certain edges $e$ of the graph. If one has test functions $f_{j}$ and $f_{j+1}$ which are concentrated at time $t=0$, one can formally indicate that the Wightman function $W_{n}\left(f_{1}, \ldots, f_{n}\right)$ should be invariant under swapping $f_{j}$ and $f_{j+1}$, by keeping most of the group elements $g_{e}$ fixed, but replacing each $g_{e}$ such that edge $e$ crosses from band $j$ to band $j+1$ with the element $\tau g_{e}$, where $\tau$ is reflection in the plane $t=0$ (Rehren discusses this substitution further in [Reh1]).

However, the above argument is not good enough for a formal proof, because the convergence arguments of $\S 5$ are valid only for functions in the Schwartz space, not for functions concentrated at $t=0$; so one has to replace this simple argument with something really quite delicate, which we do in $\S 9$, the longest and most difficult section of the paper.

In spite of its difficulty, the author feels personally that this is one of the most promising parts of the construction, and that there is real hope that one day an argu- 
ment like this may be used to prove locality for nontrivial theories. For the argument is a way by which one may seek to prove results which are hinted at when one formally equates two divergent integrals; such as one frequently encounters in this area of research.

The spectral properties of our construction are relatively easy to establish, because we are able to identify the Hamiltonian of our theory in a fairly explicit manner, in $\S 8$ (it is the restriction to the appropriate subspace of the Hamiltonian of the large Fock space mentioned above).

Well, that is the outline of our proof. We now begin the proof itself.

\section{Preliminary Definitions}

What follows is consistently done in dimension $d+1$.

This theory is intended to be based on a free theory with "smoothly smeared" mass. Thus, in the free theory we have a vacuum state $\Omega$, and some field operators $\phi_{M}(f)$, with a two point function, $C_{M}(f, g)$, given by

$$
C_{M}(f, g)=<\phi_{M}(f) \Omega, \phi_{M}(g) \Omega>=\int_{0}^{\infty} \int_{\mathbb{R}^{d}} \widehat{f}(-\mathbf{k},-L) \widehat{g}(\mathbf{k}, L) \mu\left(L^{2}-k^{2}\right) d \mathbf{k} d L,
$$

where ${ }^{-}$denotes the Fourier Transform, and the function $\mu$ is supported on $\mathbb{R}^{+}$.

By the Källén Lehmann representation theorem, this is the general form for the two point function of any theory satisfying the Wightman axioms, except that the nonnegative function $\mu$ may be replaced by a measure.

Our functions $\mu$ will always be infinitely differentiable and supported on an interval $\left[m_{0}^{2}, m_{1}^{2}\right]$, with $m_{1}>m_{0}>0$ and (we may assume when doing calculations later on, though it is not essential) $m_{1}<2 m_{0}$. We shall thus be assuming that $\mu \in C_{0}^{\infty}$. This last means that we think we can tell the one-particle states from the rest (only states with mass between $m_{0}$ and $\left.m_{1}\right)$. Our convention with regard to the Fourier Transform is that it is

$$
\widehat{f}(\mathbf{p})=\int f(\mathbf{x}) e^{-\mathrm{i} \mathbf{p} \cdot \mathbf{x}} d \mathbf{x}
$$

so the inverse Fourier transform will have a factor $(2 \pi)^{-(d+1)}$ in $d+1$ dimensions.

The theory will be obtained by performing an operation analogous (fairly!) to formal perturbation theory with a restricted set of Feynman graphs. The (finite) set of graphs involved is described in the next section. The polynomial interaction will be of form

$$
P(\phi)=\sum_{i=2}^{N} a_{i} \phi^{i}
$$

where the coefficients $a_{i}$ are real. Note that we expect our polynomial to begin with a quadratic term; linear perturbations can be done separately if desired. The "constants" involved in our theory, apart from the mass density $\mu$ and polynomial interaction $P$, are as follows: an integer $S>0$ regulates the number of graphs involved; a smoothing function $\theta \in \mathrm{S}\left(\mathrm{R}^{d+1}\right)$ "helps things to converge." [Limits as $\theta$ tends to some special class of function, e.g. a delta function, may be of interest but are not attempted in this paper. What we do with the function $\theta$ is somewhat akin to replacing the field $\phi$ with a convolved version $\phi * \theta$; the process being done with the aid of a certain averaging process over the Lorentz group, which preserves covariance and locality. We obtain 
fully local and covariant results without letting $\theta$ become singular, and our objective here is to produce good answers which converge as "obviously" as possible.] And of course, there is a real coupling constant $\lambda$. As we shall see, the Wightman function $W_{n}$ of our theory is a polynomial of degree at most $n S$ in the coupling constant $\lambda$. It is obtained by summing certain integrals associated with graphs of at most $n S$ internal vertices. We begin the definitions in Sect. 2, and conclude them in Sect. 3.

\section{2. "Banded" Graphs}

First, we apologise in advance for overuse of the letter $I$ in this paper. Roman " $\mathrm{i}$ " is used in this paper for the square root of -1 , italic " $i$ " for the index to innumerable sums; " $I_{G}$ " is a graphical $I$ ntegrand, and " $G_{\mathscr{T}}$ " is the set of $\mathscr{T}$ nternal vertices of a graph $G$.

If $G$ is a graph, we write $G_{v}$ ! for the product

$$
\prod_{v \in G} d(v) !
$$

where $d(v)$ is the degree of the vertex $v$. Should some of the edges of $G$ perhaps be multiple, we define

$$
G_{e} !=\prod_{(v, w) \in e(G)} m(v, w) !
$$

where $e(G)$ is the set of edges of $G$ and $m(v, w)$ denotes the multiplicity of an edge.

Definition 2.1. Fix an integer constant $S>0$, which will be one of the parameters of the theory.

For each $n>0$, we define a collection $G_{n}$ of labelled graphs as follows.

$\mathscr{G}_{n}$ is the collection of all graphs $G$ satisfying the following conditions:

The graph $G$ has $n$ "external" vertices labelled $F_{1}, \ldots, F_{n}$, and from zero up to $n S$ internal vertices. The internal vertices are labelled $X_{i, j}\left(i=1, \ldots, n ; 1 \leq j \leq s_{i}\right)$; where every $s_{i}=s_{i}(G) \in[0, S]\left(s_{\imath}=0\right.$ is allowed $)$. Multiple edges are allowed between (internal) vertices, but not "loops" connecting a vertex to itself.

The ith band of the graph $(i=1, \ldots, n)$ is defined to be the subgraph with vertex set $B_{\imath}=B_{\imath}(G)=\left\{F_{i}, X_{i, j}: j=1, \ldots, s_{\imath}\right\}$.

In addition, the graph $G$ must satisfy the following:

(2.1.1) The degree of every external vertex is 1 .

(2.1.2) The degree of every internal vertex is between 2 and $N$, where $N$ is the degree of the polynomial $P$ on which our theory is to be based.

(2.1.3) Every band of $G$ is a connected subgraph of $G$.

Note 2.2: If the last condition (2.1.3) were omitted there would be divergent contributions to our Wightman functions coming from graphs with one or more component disconnected from any external vertex. (2.1.3) therefore performs something akin to division by $\mathrm{Z}$ in the heuristics. Let us also emphasise that graphs in $\mathscr{G}_{n}$ are labelled graphs; the same abstract graph may have several manifestations as an element of $\mathscr{G}_{n}$.

The Wightman $n$-point function will be defined in the next section as a sum of "Feynman-like" integrals associated with each graph $G$ in $\mathrm{G}_{n}$. 


\section{The Graphical Integrals}

Let $S$ be given as in the previous section, and let us also choose, once and for all, a smoothing function $\theta \in \mathscr{S}\left(\mathbb{R}^{d+1}\right)$, a coupling constant $\lambda \in \mathbb{R}$, a polynomial interaction $P$ as in (1.2) and a smeared mass density $\mu$ as in (1.1). Note that the value $\lambda=0$ is allowed, though it just gives a Gaussian theory. Also, our theories exist for either sign of the coupling constant.

For each graph $G \in \mathscr{G}_{n}$, and each sequence $f_{1}, \ldots, f_{n}$ of functions in the Schwartz space $\mathbf{S}\left(\mathrm{R}^{d+1}\right)$, we begin to define the graphical integral $I_{G}\left(f_{1}, \ldots, f_{n}\right)$ associated with the graph $G$, which will contribute to the Wightman n-point function $W_{n}\left(f_{1}, \ldots, f_{n}\right)$ of our theory.

Definition 3.1. Given $G$ and the sequence $\mathbf{f}=f_{1}, \ldots, f_{n}$, let us choose two sequences as follows:

Let a sequence $\mathbf{p}=\left(\mathbf{p}_{e}: e \in e(G)\right)$ be given, consisting of elements of $\mathbb{R}^{d+1}$; we shall sometimes write $\mathbf{p}_{e}=\left(\mathbf{k}_{e}, L_{e}\right)\left(\mathbf{k}_{e} \in \mathbb{R}^{d}, L_{e} \in \mathbb{R}\right)$, where $L_{e}$ is the time coordinate. (Note that we intend to use bold italic $\mathbf{p}$ for individual vectors in $\mathbb{R}^{d+1}$, reserving bold roman $\mathbf{p}$ for use with vectors in larger spaces such as $\mathbb{R}^{(d+1) e(G)}$.)

Then, let a sequence $\mathbf{g}=\left(g_{e}, e \in e_{X}(G)\right)$ be given, (where $e_{X}(G)$ ("e-cross of $G$ ") is the set of edges of $G$ which connect two different bands of $G$ ) consisting of elements of the full Lorentz group $L$ on $\mathbb{R}^{d+1}$. We emphasise, time reversals are allowed in L; so also are space "rotations" with determinant -1 .

The graphical integrand $M_{G}(\mathbf{f}, \mathbf{p}, \mathbf{g})$ is the product

$$
\prod_{e \in e(G)} M_{G}^{e}
$$

where the functions $M_{G}^{e}(\mathbf{f}, \mathbf{p}, \mathbf{g})$ are as follows:

(3.1.2) If e connects $F_{i}$ to $F_{j}$ with $i<j$, then

$$
M_{G}^{e}=\mathrm{H}\left(L_{e}\right) \mu\left(\mathbf{p}_{e}^{2}\right) \widehat{f}_{j}\left(-\mathbf{p}_{e}\right) \widehat{f}_{i}\left(\mathbf{p}_{e}\right)\left|\widehat{\theta}\left(g_{e} \mathbf{p}_{e}\right)\right|^{2},
$$

where $H$ denotes the step function, $H(x)=1(x>0)$ or $0,(x \leq 0)$; and $\mathbf{p}_{e}^{2}$ is $L_{e}^{2}-k_{e}^{2}$. Here and elsewhere, we write just $g_{e} \mathbf{p}_{e}$ for the value $g_{e}\left(\mathbf{p}_{e}\right),\left(g_{e} \in \mathrm{L}, \mathbf{p}_{e} \in \mathbb{R}^{d+1}\right)$.

(3.1.3) If e connects $F_{i}$ to $X_{j, k}$, with $i<j$, then

$$
M_{G}^{e}=\mathrm{H}\left(L_{e}\right) \mu\left(\mathbf{p}_{e}^{2}\right) \widehat{f}_{i}\left(\mathbf{p}_{e}\right)\left|\widehat{\theta}\left(g_{e} \mathbf{p}_{e}\right)\right|^{2} .
$$

(3.1.4) If e connects $F_{i}$ to $X_{j, k}$, with $i>j$, then

$$
M_{G}^{e}=\mathrm{H}\left(L_{e}\right) \mu\left(\mathbf{p}_{e}^{2}\right) \widehat{f}_{i}\left(-\mathbf{p}_{e}\right)\left|\widehat{\theta}\left(g_{e} \mathbf{p}_{e}\right)\right|^{2} .
$$

(3.1.5) If e connects $F_{i}$ to $X_{i, k}$ then

$$
M_{G}^{e}=\mu\left(\mathbf{p}_{e}^{2}\right) \widehat{f}_{\imath}\left(\phi_{i}(\mathbf{p})\right)\left|\widehat{\theta}\left(\mathbf{p}_{e}\right)\right|^{2},
$$

where the linear functional $\phi_{i}(\mathbf{p})$ is as follows:

$$
\phi_{i}(\mathbf{p})=\sum_{\substack{e \text { connects Band } \\ \text { to Band } j, i<j}} \mathbf{p}_{e}-\sum_{\substack{e \text { connects Band } \\ \text { to Band } j, i>j}} \mathbf{p}_{e}
$$




$$
=\sum_{j>i} \phi_{i, j}(\mathbf{p})-\sum_{j<i} \phi_{i, j}(\mathbf{p})
$$

let us say.

(3.1.6) If e connects $X_{i, j}$ to $X_{k, l}$ with $i<k$ then

$$
M_{G}^{e}=\mathrm{H}\left(L_{e}\right) \mu\left(\mathbf{p}_{e}^{2}\right)\left|\widehat{\theta}\left(g_{e} \mathbf{p}_{e}\right)\right|^{2} .
$$

(3.1.7) If e connects $X_{i, j}$ to $X_{i, l}$ with $j<l$ then

$$
M_{G}^{e}=\mathrm{H}\left(L_{e}\right) \mu\left(\mathbf{p}_{e}^{2}\right)\left|\widehat{\theta}\left(\mathbf{p}_{e}\right)\right|^{2} .
$$

Notes on Definition 3: This is of course the fundamental definition of the paper. Note that if one ignores the smoothing functions $\theta$ and the Lorentz group elements $g_{e}$, and if one mentally prepares to integrate with respect to the momenta $\mathbf{p}_{e}$, then these expressions are not a million miles from the heuristic $C_{M}\left(f_{i}, f_{j}\right)$ for edges $e=\left(F_{i}, F_{j}\right) ; C_{M} f_{i}\left(x_{j, k}\right)$ for $e=\left(F_{i}, X_{j, k}\right)$ and $C_{M}\left(x_{i, \jmath}, x_{k, l}\right)$ for $e=\left(X_{i, j}, X_{k, l}\right)$. We should perhaps emphasise, that multiple edges are of course represented multiply in the product (3.1.1), as they are in the sequences $\mathbf{p}$ and $\mathbf{g}$.

Definition 3.2. Let us adopt the notation of (3.1) and define further linear functions $\psi_{i, j}(\mathbf{p})\left(i=1, \ldots, n ; j=1, \ldots, s_{i}\right)$ as follows. Here and elsewhere, we adopt the convention that if the edge e is internal to some band of $G$ (that is, if $e \in e(G) \backslash e_{X}(G)$ ) then the group element $g_{e}$ is deemed to be the identity. Then,

$$
\psi_{i, j}(\mathbf{p})=\psi_{\imath, j}(G, \mathbf{p}, \mathbf{g})=\sum_{e \in S^{+}} g_{e} \mathbf{p}_{e} \quad-\sum_{e \in S^{-}} g_{e} \mathbf{p}_{e}
$$

where

$$
\begin{gathered}
S^{+}=S_{i, j}^{+}(G)=\left\{e \in e(G): \text { e connects } X_{i, j} \text { to } F_{k}(\text { with } k>i) \text { or } X_{k, l}\right. \\
(\text { with } k>i, \text { or } k=i \text { and } l>j)\}
\end{gathered}
$$

and

$$
\begin{gathered}
S^{-}=S_{i, j}^{-}(G)=\left\{e \in e(G): \text { e connects } X_{i, j} \text { to } F_{k}(\text { with } k \leq i) \text { or } X_{k, l}\right. \\
(\text { with } k<i, \text { or } k=i \text { and } l<j)\}
\end{gathered}
$$

Note that $\psi_{\imath, j}$ is the total flow out of vertex $X_{i, j}$ if each edge of $G$ is given a directed flow $g_{e} \mathbf{p}_{e}$, the direction being "upwards" from $X_{i, j}$ to $X_{k, l}$ (when $i<k$, or $i=k$ and $j<l$ ), and from $X_{i, j}$ or $F_{i}$ to $F_{k}$ (when $i<k$ ); otherwise downwards. The reader will find that the flows $\phi_{i, j}$ and $\phi_{i}$ of (3.1.5) also respect this orientation. From now on we will feel free to refer to one vertex of a graph being "above" another in this sense.

Definition 3.3. The first integrated moment $M_{G, 1}(\mathbf{f}, \mathbf{g})$ is the integral

$$
\int_{\mathbb{R}^{(d+1) e(G)}} M_{G}(\mathbf{f}, \mathbf{p}, \mathbf{g})\left(\prod_{\substack{\imath=1, \ldots . n \\ j=1, \ldots, s_{\imath}}} \delta\left(\psi_{\imath, j}(G, \mathbf{p}, \mathbf{g})\right)\right) d \mathbf{p}
$$


where dp denotes volume integration over all variables $\mathbf{p}_{e}, e \in e(G) ; \delta$ denotes the $d+1$-dimensional version of the Dirac delta function; and questions of convergence are temporarily postponed.

Concerning this definition, we would like to emphasise that the delta- functions are evaluated at $\psi_{\imath, j}$, a function which depends linearly on the variables $\mathbf{p}_{e}$, but also on the variables $g_{e}$. Therefore, the variables $g_{e}$ cannot be "integrated out" to yield a Lorentz invariant version of the function $\theta$. Also, when considering behaviour around an internal vertex of one of these graphs, one should bear in mind that our integrals are not concentrated on values of $\mathbf{p}_{e}$ which add up to zero at the vertex; they are concentrated on values $g_{e} \mathbf{p}_{e}$ which add up to zero at the vertex.

Definition 3.4. The second integrated moment $M_{G, 2}(\mathbf{f})$ is (assuming convergence) the integral

$$
\int^{\left|e_{X}(G)\right|} M_{G, 1}(\mathbf{f}, \mathbf{g}) \prod_{e \in e_{X}(G)} d g_{e},
$$

where $\left|e_{X}(G)\right|$ denotes the number of edges of $G$ that cross between two different bands of $G$, and $d g$ denotes right-invariant Haar measure on the Lorentz group $L$.

[Right invariant Haar measure is left invariant as well, but it is the right invariance we need. The measure may be written (up to an arbitrary constant) as

$$
d U \frac{d v_{1} \ldots d v_{d}}{\left(\sqrt{1-v^{2}}\right)^{d+1}}
$$

where the element $g$ is written $\Lambda_{\mathbf{v}} U$, where $U$ is a space rotation and $\Lambda_{\mathbf{v}}$ a velocity boost of velocity $\mathbf{v}$, and $d U$ is (finite!) Haar measure on the space rotations.]

Definition 3.5. The graphical integral $I_{G}(\mathbf{f})$ is defined as

$$
\frac{G_{v} !}{G_{e} !}(-\lambda)^{\left|G_{\mathscr{T}}\right|} \prod_{\substack{\imath=1, \ldots . n \\ \jmath=1, \ldots s_{\imath}}} a_{d\left(X_{\imath, \jmath}\right)} M_{G, 2}(\mathbf{f})
$$

where $\lambda$ is the coupling constant, $d\left(X_{i, j}\right)$ is the degree of the vertex, $a_{d}$ is the dth coefficient of the polynomial $P$ on which our theory is based, and $\left|G_{\mathscr{T}}\right|$ is the number of internal vertices of $G$.

We will write

$$
a(G)=\prod_{\substack{i=1, \ldots . n \\ j=1, \ldots . s_{i}}} a_{d\left(X_{i, j}\right)} .
$$

Note that the factors $G_{v}$ ! in (3.5.1) may be regarded as arbitrary (one could compensate for their absence by changing the polynomial, though one's conviction that there ought to be factors of 4 ! floating around a $\phi^{4}$ theory dies hard). However, the factors $G_{e}$ ! are essential; the proof of positivity will not work without them.

Definition 3.6. The Wightman n-point function $W_{n}\left(f_{1}, \ldots, f_{n}\right)$ is equal to

$$
\sum_{G \in \mathscr{G}_{n}} I_{G}(\mathbf{f})
$$

The constant $W_{0}$ is defined equal to 1. 
It is now our business to show that $W_{n}(\mathbf{f})$ is indeed the $n$-point function of a scalar field theory.

\section{Finiteness}

The object of this section is to prove the convergence of all doubtful integrals in Sect. 3.

Lemma 4.1. The integrand $M_{G}(\mathbf{f}, \mathbf{p}, \mathbf{g})$ is equal to

$$
\prod_{i=1}^{n} \widehat{f}_{i}\left(\phi_{i}(\mathbf{p})\right) N_{G}(\mathbf{p}, \mathbf{g}),
$$

where $N_{G}$ does not depend on $\mathbf{f}$; and for fixed $\mathbf{g}$, it is in the Schwartz space $\mathscr{S}\left(\mathbb{R}^{(d+1) e(G)}\right)$ as a function of $\mathbf{p .}$ In fact,

$$
N_{G}(\mathbf{p}, \mathbf{g})=\prod_{e \in e(G)} N_{G}^{e}(\mathbf{p}, \mathbf{g})
$$

where $N_{G}^{e}$ is as follows:

(4.1.3) If e connects $F_{i}$ or $X_{i, l}$ to $F_{j}$ or $X_{j, k}, i \neq j$, then

$$
N_{G}^{e}=\mathrm{H}\left(L_{e}\right) \mu\left(\mathbf{p}_{e}^{2}\right)\left|\widehat{\theta}\left(g_{e} \mathbf{p}_{e}\right)\right|^{2} .
$$

(4.1.4) If e connects $F_{i}$ to $X_{i, k}$, then

$$
N_{G}^{e}=\mu\left(\mathbf{p}_{e}^{2}\right)\left|\widehat{\theta}\left(g_{e} \mathbf{p}_{e}\right)\right|^{2} .
$$

Proof . Looking at (3.1.2-7) we see that each $N_{G}^{e}$ is an expression similar to $M_{G}^{e}$ but with all factors $\widehat{f}_{i}(\mathbf{q})$ removed. Since, by (3.1.1) $M_{G}$ is the product of all the $M_{G}^{e}$, in order to establish (4.1.1) we need to show one thing:

(1) There is, for each $i=1 . . n$, a unique factor $\widehat{f}_{i}(\mathbf{q})$ among the $M_{G}^{e}$; and invariably we have $\mathbf{q}=\phi_{i}(\mathbf{p})$.

Proof of (1): By (2.1.1) the degree of vertex $F_{i}$ is 1 so there is a unique edge $e$ incident at $F_{i}$. This edge will (by (3.1.2-5)) contribute a single factor $\widehat{f}_{i}(\mathbf{q})$ to $\mathbf{M}$ for some $\mathbf{q}$; let us show that $\mathbf{q}=\phi_{i}(\mathbf{p})$. If $e=\left(F_{i}, X_{\imath, k}\right)$ for some $k$, this is the definition (3.1.5). If $e=\left(F_{i}, F_{j}\right)$ for some $j>i$ then $\mathbf{q}=\mathbf{p}_{e}$ by (3.1.2), as is also the case if $e=\left(F_{i}, X_{\jmath, k}\right)$ with $j>i$ by (3.1.3). By (2.1.3) Band $i$ of $G$ is connected; since $F_{i}$ is not (in these two cases) connected to any other vertex in Band $i$, we conclude that $F_{i}$ is the only vertex in Band $i$ (i.e. $s_{\imath}=0$ ); hence the only edge out of Band $i$ is $e$ , going to Band $j$ with $j>i$; therefore $\phi_{i}(\mathbf{p})=\mathbf{p}_{e}=\mathbf{q}$. Likewise if $e=\left(F_{i}, F_{j}\right)$ or $\left(F_{i}, X_{j, k}\right)$ with $j<i$, we still have $s_{i}=0$ by connectedness of Band $i$, only this time the edge $e$ goes downwards to Band $j, j<i$, so $\phi_{i}(\mathbf{p})=-\mathbf{p}_{e}$; and this agrees with the value $\mathbf{q}$ found in (3.1.2,3). Thus (1) is proved. Thus we have established (4.1.1). That $N_{G}$ does not depend on $\mathbf{f}$ is obvious from (4.1.3-7). We claim that as a function of $\mathbf{p}$ it is in the Schwartz space; because for each edge $e$ there is a factor $\left|\widehat{\theta}\left(g_{e} \mathbf{p}_{e}\right)\right|^{2}$; and $\widehat{\theta} \circ \mathbf{g} \in \mathscr{S}\left(\mathbb{R}^{d+1}\right)$ because $\theta$ is; and the other factors involved are all suitably smooth because $\mu \in \mathscr{S}(\mathbb{R})$ is supported on $\left[m_{0}^{2}, m_{1}^{2}\right]$ with $m_{0}>0$. Thus our lemma is proved. 
Note: This is a classic example of probabilistic mass "making things easier."

Lemma 4.2. The first integrated moment $M_{G, 1}(\mathbf{f}, \mathbf{g})$ exists and is equal to

$$
\int_{\mathbb{R}(d+1) e(G)}\left(\prod_{i=1}^{n} \widehat{f}_{i}\left(\phi_{i}(\mathbf{p})\right)\right)\left(\prod_{\substack{i=1, \ldots . n \\ \jmath=1, \ldots s_{i}}} \delta\left(\psi_{i, j}(\mathbf{p})\right)\right) N_{G}(\mathbf{p}, \mathbf{g}) d \mathbf{p}
$$

(where $\delta$ is the $d+1$-dimensional Dirac delta-function). It is a continuous multilinear function of $f_{1}, \ldots, f_{n}$.

Before proving the lemma, let us note that there is some modest similarity to a Feynman integral here. If we compare the integral with

$$
\int_{\mathbb{\mathbb { R }}(d+1) e(G)}\left(\prod_{\imath=1}^{n} \widehat{f}_{i}\left(\phi_{i}(\mathbf{p})\right)\right)\left(\prod_{\substack{i=1, \ldots \ldots n \\ j=1, \ldots, s_{\imath}}} \delta\left(\psi_{i, j}(\mathbf{p})\right)\right)\left(\prod \frac{1}{\mathbf{p}_{e}{ }^{2}-m^{2}}\right) d \mathbf{p}
$$

then the resemblance becomes quite reasonable, since the function $N_{G}$ does indeed consist of a product of a lot of functions of $p_{e}^{2}$, together with signs of the $L_{e}$ which are associated with the orientation ("bands" etc.) of the graph $G$.

Proof . That (4.2.1) is the right expression provided it converges, is obvious from (3.3.1) and (4.1).

By (4.2.1) we are integrating a function in the Schwartz space next to a product of various delta-functions in linear combinations of the variables $\mathbf{p}_{e}$ under integration. It is therefore necessary and sufficient that we prove these combinations linearly independent.

Let $\mathbf{e}_{0}, \ldots, \mathbf{e}_{d}$ be the unit vector basis of $\mathbb{R}^{d+1}$. We must show that (for fixed $\mathbf{g}$ ) the $\left|G_{\mathscr{T}}\right|(d+1)$ linear functionals on $\mathbb{R}^{(d+1) e(G)}$ given by

$$
\mathbf{p} \rightarrow \psi_{i, j}(\mathbf{p}) \cdot \mathbf{e}_{k}
$$

$(k=0, \ldots, d ; e \in e(G))$ are linearly independent. Give each edge of $\mathrm{G}$ a flow $g_{e} \mathbf{p}_{e}$ in the direction given in (3.2). Since the $g_{e}$ are invertible, let us write $\mathbf{q}_{e}=g_{e} \mathbf{p}_{e}$ and show that the above functionals are independent as functions of $\mathbf{q}$. Now, the $\psi_{i, j}$ are the total q-flows into vertices $X_{i, j}$; so it is enough if for each fixed k, we show that the functionals $\psi_{i, j}(\mathbf{p}) \cdot \mathbf{e}_{k}\left(i=1, \ldots . n ; j=1, \ldots . s_{i}\right)$ are independent. But real-valued flows on graphs are well known; within any component $H$ of $G$, say involving m vertices, it is known that any $m-1$ of those flows are linearly independent. But every component of $G$ is a union of some of the bands by (2.1.3); so it contains at least one vertex $F_{i}$; hence the flows out of vertices $X_{i, j}$ are indeed independent, because they never include all the flows out of vertices in a given component. That the integral (4.2.1) is a continuous multilinear functional of the $f_{i}$ is then obvious, so our lemma is proved.

Lemma 4.3. The second integrated moment exists, and $M_{G, 2}(\mathbf{f})$ is given by the integral

$$
\int^{\left|e_{X}(G)\right|} \int_{\mathbb{R}^{(}(d+1) e(G)}\left(\prod_{i=1}^{n} \widehat{f}_{i}\left(\phi_{i}(\mathbf{p})\right)\right)\left(\prod_{\substack{r=1, \ldots . n \\ j=1, \ldots, s_{i}}} \delta\left(\psi_{i, j}(\mathbf{p})\right)\right) N_{G}(\mathbf{p}, \mathbf{g}) d \mathbf{p} d \mathbf{g}
$$


where d $\mathbf{g}$ denotes the product of $\left|e_{X}(G)\right|$ copies of Haar measure on the Lorentz group L. This integral, moreover, is absolutely convergent (subject to the usual conventions about removing the delta functions in favour of integrating over rather fewer of the variables $\left.\mathbf{p}_{e}\right)$, and it is a continuous multilinear functional of the $f_{i}$.

Proof. That (4.3.1) will give the second moment if it converges is a consequence of Lemma 4.2. Removal of the delta- functions from (4.3.1) can be done in the following helpful way. By (2.1.3) each band of $G$ is connected; so if $s_{i}>0$ for some $i$, we can choose ourselves a spanning tree $T_{i}$ for band $\mathrm{i}$ (that is, a graph $T_{i}$ on vertex set $\left\{F_{i}, X_{i, j} ; j=1, \ldots s_{\imath}\right\}$ with $e\left(T_{\imath}\right) \subseteq e(G)$ and $e\left(T_{\imath}\right)$ a minimal set such that $T_{i}$ is connected), which can be rooted at vertex $F_{i}$ (that is, the edges systematically directed away from the root vertex $F_{i}$ ). Choose such a tree for each band, and let $\bar{T}$ be the union of all the edges involved in the $T_{i}$. For each edge $e \in \bar{T}$ let $X_{i, j} \quad((i, j)=(i(e), j(e))$ be the unique upper vertex of edge $e$ (which is away from the root vertex $F_{i}$ ). Given a sequence $\mathbf{g} \in L^{\left|e_{X}(G)\right|}$ we define a unique linear map $\eta=\eta_{\mathbf{g}}$ on $\mathbb{R}^{(d+1) e(G)}$ in the following implicit manner: we write $\eta(\mathbf{q})=\eta\left(\left(\mathbf{q}_{e}\right)_{e \in e(G)}\right)=\left(\mathbf{p}_{e}\right)_{e \in e(G)}$, where

$$
\mathbf{p}_{e}= \begin{cases}\mathbf{q}_{e}, & \text { if } e \in e(G) \backslash \bar{T}, \\ \mathbf{q}_{e}+\sum_{e^{\prime} \in S_{\imath, j}^{+}(G)} g_{e^{\prime}} \mathbf{p}_{e^{\prime}}-\sum_{e^{\prime} \in S_{i, j}^{-}(G) \backslash\{e\}} g_{e^{\prime}} \mathbf{p}_{e^{\prime}}, & \text { if } e \in S_{i, j}^{-}(G), \\ \mathbf{q}_{e}+\sum_{e^{\prime} \in S_{\imath, j}^{-}(G)} g_{e^{\prime}} \mathbf{p}_{e^{\prime}}-\sum_{e^{\prime} \in S_{i, j}^{+}(G) \backslash\{e\}} g_{e^{\prime}} \mathbf{p}_{e^{\prime}}, & \text { if } e \in S_{i, j}^{+}(G)\end{cases}
$$

where $(i, j)=(i(e), j(e))\}$; and once again $g_{e^{\prime}}$ is deemed to be the identity if $e^{\prime} \notin$ $e_{X}(G)$.

We claim that there is indeed a unique linear map with these properties. For if $e \in T_{i}$ is an edge at maximum distance from the root $F_{2},(4.3 .2)$ gives $\mathbf{p}_{e}=$ $\mathbf{q}_{e}+\sum \pm g_{e^{\prime}} \mathbf{q}_{e^{\prime}}$ since no other edge in $T_{i}$ is incident at $X_{i, j}$. If $e \in T_{i}$ is at distance one less than the maximum, (4.3.2) then gives $\mathbf{q}_{e}$ in terms of the $\mathbf{q}_{e}(e \notin \bar{T})$ and the $\mathbf{p}_{e}$ ( $e$ at maximum distance from $F_{i}$ ). Continuing the process down to the root vertex we find that the map does indeed exist and is unique. Note that it also has determinant equal to one because its matrix is upper triangular with diagonal entries equal to 1 if we take a basis of $\mathbb{R}^{(d+1) e(G)}$ consisting of vectors as follows.

The "natural unit vectors" $\mathbf{u}_{e, i}(e \in e(G), i=0, \ldots . d)$ in $\mathbb{R}^{(d+1) e(G)}$ are sequences $\mathbf{q}=\left(\mathbf{q}_{f}\right)_{f \in e(G)}$ such that $\mathbf{q}_{f}=0$ unless $f=e$, in which case $\mathbf{q}_{f}=\mathbf{e}_{\imath}$, the $i$ th unit vector in $\mathbb{R}^{d+1}$. We choose a basis of $\mathbb{R}^{(d+1) e(G)}$ consisting of natural unit vectors $\mathbf{u}_{e, i}$ in an order with the following properties: first, unit vectors $\mathbf{u}_{e, i}$ for edges $e \notin \bar{T}$ appear $\left(d+1\right.$ of them for each such edge), then vectors $\mathbf{u}_{e, i}$ appear for the edges in $\bar{T}$ at maximum distance from their roots, then for those distant one less, and so on. In view of (4.3.2) it is clear that the matrix of $\eta$ will be upper triangular with respect to such a basis, with diagonal elements equal to 1 .

If we make the substitution $\mathbf{p}=\eta_{\mathbf{g}}(\mathbf{q})$ in (4.3.1), the delta functions of (4.3.1) are precisely $\prod_{e \in \bar{T}} \boldsymbol{\delta}\left(\mathbf{q}_{e}\right)$. So, if we define a vectorspace $V_{T}$ isomorphic to $\mathbb{R}^{(d+1)(e(G) \backslash e(\bar{T}))}$ consisting of sequences $\left(\mathbf{q}_{e}\right)_{e \in e(G) \backslash \bar{T}}$ in $\mathbb{R}^{d+1}$, and if $\epsilon$ denotes the map $V_{T} \rightarrow$ $\mathbb{R}^{(d+1) e(G)}$ with

$$
\epsilon(\mathbf{q})=\mathbf{q}^{\prime}, \mathbf{q}_{e}{ }^{\prime}=\left\{\begin{array}{l}
\mathbf{0}, e \in \bar{T} \\
\mathbf{q}_{e}, e \in e(G) \backslash \bar{T},
\end{array}\right.
$$

and if $\zeta_{\mathbf{g}}$ denotes the map $\eta_{\mathbf{g}} \circ \epsilon$ (4.3.4), we find (4.3.1) is equal to 


$$
\int^{\left|e_{X}(G)\right|} \int_{V_{T}}\left(\prod_{i=1}^{n} \widehat{f}\left(\phi_{i}\left(\zeta_{\mathbf{g}}(\mathbf{q})\right)\right)\right) N_{G}\left(\zeta_{\mathbf{g}}(\mathbf{q}), \mathbf{g}\right) d \mathbf{q} d \mathbf{g} .
$$

Moreover, since $\phi_{i}(\mathbf{p})$ depends only on momenta $\mathbf{p}_{e}$ for $e \in e_{X}(G)$, this is also equal to

$$
\int^{\left|e_{X}(G)\right|} \int_{V_{T}}\left(\prod_{i=1}^{n} \widehat{f}\left(\phi_{i}(\epsilon(\mathbf{q}))\right)\right) N_{G}\left(\zeta_{\mathbf{g}}(\mathbf{q}), \mathbf{g}\right) d \mathbf{q} d \mathbf{g} .
$$

Let us now take (4.3.5) and perform all the $d \mathbf{g}$ integrations - or rather, estimate them! Looking at (4.1) we find that if $e \in e_{X}(G)$ there is invariably a factor $\widehat{\theta}\left(g_{e} \mathbf{p}_{e}\right)$ in $N_{G}$. Also, if $e \in e(G) \backslash e_{X}(G) \backslash \bar{T}$, there is a factor $|\widehat{\theta}(\mathbf{p})|^{2}$ in $N_{G}$. So since $\zeta_{\mathbf{g}}(\mathbf{q})_{e}=\mathbf{q}_{e}$ for $e \notin \bar{T}$, we may write

$$
N_{G}\left(\zeta_{\mathbf{g}}(\mathbf{q}), \mathbf{g}\right)=N_{G}^{\prime}\left(\zeta_{\mathbf{g}}(\mathbf{q}), \mathbf{g}\right) \cdot \prod_{e \in e(G) \backslash e_{X}(G) \backslash \bar{T}}\left|\widehat{\theta}\left(\mathbf{q}_{e}\right)\right|^{2} \cdot \prod_{e \in e_{X}(G)}\left|\widehat{\theta}\left(g_{e} \mathbf{q}_{e}\right)\right|^{2},
$$

where the function $N_{G}^{\prime}$ is composed of all the other factors involved in $N_{G}$, and the main thing we need know about $N_{G}^{\prime}$ is that it is uniformly bounded independent of $\mathbf{q}$ and $\mathbf{g}$; let's say $\left|N_{G}^{\prime}\right| \leq C_{1}$. Also, $N_{G}^{\prime}=0$ unless every $\mathbf{q}_{e}, e \in e_{X}(G)$ is time-like with $\mathbf{q}_{e}^{2} \in\left[m_{0}^{2}, m_{1}^{2}\right]$. Write then $\operatorname{supp} N_{G}^{\prime}$ for the set of $\mathbf{q} \in V_{T}$ such that there is any $\mathbf{g}$ with $N_{G}^{\prime}(\mathbf{q}, \mathbf{g})$ nonzero. We find that for all $\mathbf{q} \in \operatorname{supp} N_{G}^{\prime}$,

$$
\begin{gathered}
\int^{\left|e_{X}(G)\right|}\left|N_{G}\left(\zeta_{\mathbf{g}}(\mathbf{q}), \mathbf{g}\right)\right| d \mathbf{g} \leq \\
C_{1} \cdot\left[\sup _{\mathbf{q}^{2} \in\left[m_{0}^{2}, m_{1}^{2}\right]} \int|\widehat{\theta}(g \mathbf{q})|^{2} d g\right]^{\left|e_{X}(G)\right|} \cdot \prod_{e \in e(G) \backslash e_{X}(G) \backslash \bar{T}}\left|\widehat{\theta}\left(\mathbf{q}_{e}\right)\right|^{2},
\end{gathered}
$$

Let us evaluate the inner supremum. Because $d g$ is Haar measure, the inner integral depends only on $\mathbf{q}^{2}$ so let's assume $\mathbf{q}=(m, \mathbf{0})$ for some $m \in\left[m_{0}, m_{1}\right]$, the nonzero coordinate being the time coordinate. This assumption is reasonable because at all other values of $\mathbf{q}^{2}$, the factors $\mu\left(\mathbf{q}^{2}\right)$ in $N_{G}$ ensure that we are not in supp $N_{G}^{\prime}$. If $g=\Lambda_{\mathbf{v}} U$ as in (3.4.1), and we write $\|x\|^{2}$ for the positive definite $x_{1}^{2}+\ldots+x_{d}^{2}+x_{0}^{2}$, then $\|g \mathbf{q}\|^{2}=m^{2}\left(1+v^{2}\right) /\left(1-v^{2}\right)$. Now $\theta \in \mathscr{S}\left(\mathbb{R}^{d+1}\right)$ so as $v \rightarrow 1$ the integrand is $o\left(\left(1-v^{2}\right)^{\alpha}\right)$ for any $\alpha>0$; in view of the formula (3.4.1) for $d g$, it is evident that the supremum is finite, say equal to $C_{2}$. Then,

$$
\int^{\left|e_{X}(G)\right|}\left|N_{G}\left(\zeta_{\mathbf{g}}(\mathbf{q}), \mathbf{g}\right)\right| d \mathbf{g} \leq C_{1} C_{2}^{\left|e_{X}(G)\right|} . \prod_{e \in e(G) \backslash e_{X}(G) \backslash \bar{T}}\left|\widehat{\theta}\left(\mathbf{q}_{e}\right)\right|,
$$

hence the magnitude of (4.3.1) is at most

$$
C_{1} C_{2}^{\left|e_{X}(G)\right|} \cdot \int^{n} \int_{\text {supp } N_{G}} \prod_{i=1}^{n}\left|\widehat{f}_{i}\left(\phi_{i}(\epsilon(\mathbf{q}))\right)\right| \cdot \prod_{e \in e(G) \backslash e_{X}(G) \backslash \bar{T}}\left|\widehat{\theta}\left(\mathbf{q}_{e}\right)\right| d \mathbf{q} .
$$

Now, we have used the notation $\mathbf{q}_{e}^{2}$ for the indeterminate $L_{e}^{2}-k_{e}^{2}$, where $\mathbf{q}_{e}=\left(\mathbf{k}_{e}, L_{e}\right)$. Let us now write $\left\|\mathbf{q}_{e}\right\|$ for the positive definite $\left(L_{e}^{2}+k_{e}^{2}\right)^{1 / 2}$. If $e$ is an edge which crosses between two different bands, $N_{G}^{\prime}=0$ unless $\mathbf{q}_{e}$ is timelike with positive time- 
coordinate $L_{e}$. So, throughout supp $N_{G}^{\prime}$, we may consider the flow into the topmost band (Band n) as follows: for all $\mathbf{q} \in \operatorname{supp} N_{G}^{\prime}$,

$$
\left\|\phi_{n}(\epsilon(\mathbf{q}))\right\| \geq\left|\phi_{n}(\epsilon(\mathbf{q})) \cdot \mathbf{e}_{0}\right|=\sum_{\substack{\text { entersband } n \\ \text { from "below" }}}\left|L_{e}\right| \geq \frac{1}{\sqrt{2}} \sum_{e / n}\left\|\mathbf{q}_{e}\right\| .
$$

(Here $\mathbf{e}_{0}$ is the time coordinate, we use the fact that all the $L_{e}$ have the same sign, and $e \nearrow k$ means " $e$ enters Band $\mathrm{k}$ from below.") Likewise, $e \searrow k$ means " $e$ enters Band $\mathrm{k}$ from above." The last inequality holds because, after all, $\mathbf{q}_{e}$ is timelike. For other bands we get similar but slightly more complicated estimates:

$$
\begin{gathered}
\left\|\phi_{n-1}(\epsilon(\mathbf{q}))\right\| \geq\left|\phi_{n-1}(\epsilon(\mathbf{q})) \cdot \mathbf{e}_{0}\right|=\left|\sum_{e \nearrow n-1} L_{e}-\sum_{e \searrow n-1} L_{e}\right| \\
\geq \sum_{e \nearrow n-1} L_{e}-\sum_{e \searrow n-1} L_{e} \geq \sum_{e \nearrow n-1} L_{e}-\sum_{e \nearrow n} L_{e} \geq \frac{1}{2} \sum_{e \nearrow n-1}\left\|\mathbf{q}_{e}\right\|-\sum_{e \nearrow n}\left\|\mathbf{q}_{e}\right\|,
\end{gathered}
$$

and generally,

$$
\left\|\phi_{k}(\epsilon(\mathbf{q}))\right\| \geq \frac{1}{2} \sum_{e \nearrow k}\left\|\mathbf{q}_{e}\right\|-\sum_{l=k+1}^{n} \sum_{e \nearrow l}\left\|\mathbf{q}_{e}\right\|
$$

for all $k=2, \ldots . n, \mathbf{q} \in \operatorname{supp} N_{G}^{\prime}$. These incredibly crude estimates become quite powerful when we use the fact that the $f_{i}$ are in the Schwartz space. Summing (4.3.7) for $k=2$ to $n$ with factors $4^{k}$ attached, we get

$$
\begin{aligned}
\sum_{k=2}^{n} 4^{k}\left\|\phi_{k}(\epsilon(\mathbf{q}))\right\| & \geq \sum_{k=2}^{n} \sum_{e \nearrow k}\left\|\mathbf{q}_{e}\right\|\left\{\frac{1}{2} \cdot 4^{k}-4^{k-1}-4^{k-2}-\ldots-4^{2}\right\} \\
& \geq \sum_{k=2}^{n} \sum_{e \nearrow k}\left\|\mathbf{q}_{e}\right\|=\sum_{e \in e_{X}(G)}\left\|\mathbf{q}_{e}\right\| .
\end{aligned}
$$

Because $f_{1}, \ldots, f_{n}$ are in $\mathscr{S}\left(\mathbb{R}^{d+1}\right)$ for each $\alpha>0$ there is a $C_{3}=C_{3}(\alpha)$ such that

$$
\begin{gathered}
\prod_{i=1}^{n}\left|\widehat{f}_{i}\left(\phi_{i}(\epsilon(\mathbf{q}))\right)\right| \leq C_{3}(\alpha)\left(1+\sum_{k=2}^{n} 4^{k}\left\|\phi_{k}(\epsilon(\mathbf{q}))\right\|\right)^{-\alpha} \\
\leq C_{3}(\alpha)\left(1+\sum_{e \in e_{X}(G)}\left\|\mathbf{q}_{e}\right\|\right)^{-\alpha}
\end{gathered}
$$

for any $\mathbf{q} \in \operatorname{supp} N_{G}^{\prime}$. Returning to (4.3.6) we find that for each $\alpha>0,(4.3 .1)$ is at most

$$
C_{1} \cdot C_{2}^{\left|e_{X}(G)\right|} \cdot C_{3}(\alpha) \int_{\text {supp } N_{G}}\left(1+\sum_{e \in e_{X}(G)}\left\|\mathbf{q}_{e}\right\|\right)^{-\alpha} \cdot\left(\prod_{e \in e(G) \backslash e_{X}(G) \backslash \bar{T}}\left|\widehat{\theta}\left(\mathbf{q}_{e}\right)\right|\right) d \mathbf{q}
$$


which (since $\mathbf{q}$ has a "free variable" $\mathbf{q}_{e}$ only for $e \in e(G) \backslash \bar{T}$, and $\theta$ is in $\mathscr{S}\left(\mathbb{R}^{d+1}\right)$ ) is, for suitably large $\alpha$, a convergent integral. So (4.3.1) converges; in view of the uniformity of estimate (4.3.8) it is indeed a continuous multilinear functional of $f_{1}, \ldots, f_{n}$. Thus the lemma is proved.

Corollary 4.4. The functions $W_{n}\left(f_{1}, \ldots, f_{n}\right)$ exist and are continuous multilinear functions of $f_{1}, \ldots, f_{n}$.

Proof . (3.5) and (3.6) give $W_{n}(\mathbf{f})$ as a finite linear combination of the moments $M_{G, 2}(\mathbf{f})$.

Having proved the existence of our functions $W_{n}(\mathbf{f})$, the next thing to do is to prove positivity in the sense of the Wightman reconstruction theorem [SW, p. 117 ]. In fact the rest of this paper will very much follow the lines of the reconstruction theorem except that, rather than proving the cluster decomposition property, we prove vacuum uniqueness directly; and our assertion of positivity is so phrased that Hermiticity comes as a trivial consequence rather than requiring another proof. Positivity is the topic of the next section.

\section{Positivity}

The aim of this section is to take the functions $W_{n}$ defined in $\S 3$, and show that there is a Hilbert space $\mathscr{H}$ containing a vector $\Omega$ (the vacuum) and, for each $n \geq 0$ and $f_{1}, \ldots, f_{n}$ in $\mathscr{S}\left(\mathbb{R}^{d+1}\right)$, a vector $\phi_{M}\left(f_{1}, \ldots, f_{n}\right) \Omega$, such that when $n=0$ the vector is $\Omega$ itself, and for all $n$ and $m$ the inner product

$$
<\phi_{M}(\mathbf{f}) \Omega, \phi_{M}(\mathbf{g}) \Omega>=W_{n+m}\left(g_{m}, g_{m-1}, \ldots, g_{1}, f_{1}, f_{2}, \ldots, f_{n}\right) .
$$
$\mathscr{H}$.

It is therefore natural to start by defining the (slightly non-obvious) Hilbert space

Definition 5.1. Let $U$ denote $\mathbb{R}^{d+1} \times L$, where $L$ is the Lorentz group as before. Elements of $U$ will typically be written $(\mathbf{q}, \eta)$. For each $n>0$ we equip the cartesian product $U^{n}$ with the product of $n$ copies of the measure $d \mathbf{q} \times d \eta$, where $d \mathbf{q}$ is Lebesgue measure on $\mathbb{R}^{d+1}$ and $d \eta$ is Haar measure on $L$. We then write $\mathscr{H}_{n}$ for the Hilbert space $L_{2}\left(U^{n}\right)$, and we write $\mathscr{H} \mathscr{C}_{0}=\mathbb{C}$. Our Hilbert space $\mathscr{H}$ is the $L_{2}$-direct sum

$$
\mathscr{H}_{0} \oplus \mathscr{H}_{1} \oplus \mathscr{H}_{2} \oplus \ldots
$$

where, if $c \in \mathbb{C}$ and $f_{i} \in \mathscr{H}_{i}$, the norm of the sequence $\left(c, f_{1}, f_{2}, f_{3}, \ldots\right)$ is

$$
\left(|c|^{2}+\sum_{n=1}^{\infty}\left\|f_{n}\right\|^{2}\right)^{1 / 2}
$$

We will regard each $\mathscr{H}_{n}$ as a subspace of $\mathscr{H}$ in the obvious way. We will now make some definitions analogous to those of $\S \S 2$ and 3. When the definitions are complete, the reader will find that the vectors $\phi_{M}(\mathbf{f}) \Omega \in \mathscr{H}$ that we have defined are in fact symmetric under permutation of the n copies of $U$ that underlie each space $\mathscr{H}_{n}=L_{2}\left(U^{n}\right)$. The vectors may thus be regarded as elements of a symmetric tensor product, as one would expect with a scalar field theory. The vectors $\phi_{M}(\mathbf{f}) \Omega$ are thus embedded in a rather large "Fock subspace" of $\mathrm{H}$; the vectors $\phi_{M}(\mathbf{f}) \Omega$ are far from 
dense, even in the subspace, so in $\S 8$ we restrict to a subspace of $\mathscr{H}$ in which they are dense.

Definition 5.2. Let the constant $S$ be given, and the polynomial $P$ on which the theory is based, and an integer $n>0$. The collection $\mathscr{G}_{n, p}$ of partial graphs is the collection of pairs $\left(G, \delta_{G}\right)$ where $G$ is a graph on vertex set $\left\{F_{i}, i=1, \ldots . n\right\} \cup\left\{X_{i, j}, i=1 . . n, j=\right.$ $\left.1 . . s_{\imath}\right\}$ with each $s_{\imath} \in\{0,1, \ldots S\}$; and $\delta_{G}$ is a function from the set $\{1,2, \ldots \Delta(G)\}$ to the vertices of $G$, and $\Delta(G) \in \mathbb{Z}, 0 \leq \Delta(G)$. In addition, the following conditions must hold:

(5.2.1) If $F_{i}$ is an external vertex, then

$$
d\left(F_{i}\right)+\left|\delta_{G}^{-1}\left\{F_{i}\right\}\right|=1,
$$

where $d(v)$ is the degree of the vertex $v$.

(5.2.2) If $X_{i, j}$ is an internal vertex, then

$$
d\left(X_{i, j}\right)+\left|\delta_{G}^{-1}\left\{X_{i, j}\right\}\right| \in\{2,3, \ldots, N\}
$$

where $N=\operatorname{deg} P$.

(5.2.3) Every "band" of $G$ (that is, each subgraph on vertex set $\left\{F_{i}\right\} \cup\left\{X_{i, j}\right\}$ for some fixed $i$ ) is connected.

Note. The idea of this is plainly that $G$ is the "top half" of a graph associated with the Wightman function $W_{n+m}\left(g_{m}, g_{m-1}, \ldots, g_{1}, f_{1}, f_{2}, \ldots, f_{n}\right)$. For $v \in G$ we will write $d_{+}(v)$ for the sum

$$
d(v)+\left|\delta_{G}^{-1}(v)\right|
$$

Note also that the conditions (5.2.1) and (5.2.2) imply an upper bound (say, $n N S$ ) on the integer $\Delta$, so that our set $\mathscr{G}_{n, p}$ is finite, just as $\mathscr{G}_{n}$ is.

Definition 5.3. Let functions $f_{1}, \ldots, f_{n}$ be given, and $\left(G, \delta_{G}\right) \in \mathscr{G}_{n, p}$. Let sequences $\mathbf{p}=\left(p_{e}: e \in e(G)\right)$, and $\gamma=\left(\gamma_{e}: e \in e_{X}(G)\right)$ be given, in $\mathbb{R}^{d+1}$ and $L$ respectively. Writing $\Delta=\Delta(G)$ we define a function $M_{G}^{\sim}(\mathbf{f}, \mathbf{p}, \gamma)$ on $U^{\Delta}$ as follows:

$$
M_{G}^{\sim}(\mathbf{f}, \mathbf{p}, \gamma)\left[\mathbf{q}_{1}, \eta_{1} ; \mathbf{q}_{2}, \eta_{2} ;, \ldots, \mathbf{q}_{\Delta}, \eta_{\Delta}\right]=\prod_{e \in e(G)} M_{G}^{\sim e} \cdot \prod_{\imath=1}^{\Delta} M_{G}^{\sim}{ }^{(i)}
$$

where the functions $M_{G}^{\sim e}(\mathbf{f}, \mathbf{p}, \gamma)[\mathbf{q}, \boldsymbol{\eta}]$ and $M^{\sim}{ }_{G}^{(i)}(\mathbf{f}, \mathbf{p}, \gamma)[\mathbf{q}, \boldsymbol{\eta}]$ are as follows. (5.3.2) If the edge e joins $F_{i}$ to $X_{i, k}$, then $M_{G}^{\sim e}=\mu\left(\mathbf{p}_{e}^{2}\right) \widehat{f}_{i}(\psi)\left|\widehat{\theta}\left(\mathbf{p}_{e}\right)\right|^{2}$, where

$$
\psi=\phi_{i}(\mathbf{p})-\sum_{\delta_{G}(j) \in B_{i}(G)} \mathbf{q}_{j}
$$

The linear function $\psi$ will sometimes be called $\phi_{i}^{\sim}(\mathbf{p}, \mathbf{q})$.

(5.3.3) In any case other than (5.3.2), the function $M_{G}^{\sim}$ is a constant (i.e., a function independent of $\mathbf{q}$ and $\boldsymbol{\eta})$ identical to the value $M_{G}^{e}(\mathbf{f}, \mathbf{p}, \gamma)$ specified in (3.1.2,3,4,6,7), where the symbol $\mathbf{g}$ is used instead of $\gamma$.

(5.3.4) If $\delta_{G}(i)$ is an external vertex $F_{j}$, then $M^{\sim}{ }_{G}{ }^{(i)}=\mathrm{H}\left(L_{i}\right) \sqrt{\mu\left(\mathbf{q}_{i}^{2}\right)} \widehat{f}_{j}\left(-\mathbf{q}_{i}\right) \widehat{\theta}\left(\eta_{i} \mathbf{q}_{i}\right)$, where $L_{i}$ denotes the time coordinate of $\mathbf{q}_{i}$.

(5.3.5) If $\delta_{G}(i)$ is an internal vertex $X_{\jmath, k}$, then $M_{G}^{\sim}{ }^{(i)}=\mathrm{H}\left(L_{\imath}\right) \sqrt{\mu\left(\mathbf{q}_{\imath}^{2}\right)} \widehat{\theta}\left(\eta_{i} \mathbf{q}_{i}\right)$. 
Note that if $\Delta=0$, the function $M^{\sim}{ }_{G}$ is of course a constant, as required; indeed, the said constant is none other than $M_{G}(\mathbf{f}, \mathbf{p}, \gamma)$ as in (3.1).

It is worth while noting that in case (5.3.4), the value $-\mathbf{q}_{i}$ is equal to $\phi^{\sim}{ }_{i}(\mathbf{p}, \mathbf{q})$, because the vertex $F_{i}$ cannot be connected to any other vertex of $G$ and so $s_{i}=0$ by (5.2.3). So, echoing (4.1), we may write

$$
M_{G}^{\sim}(\mathbf{f}, \mathbf{p}, \gamma)=\left(\prod_{i=1}^{n} \widehat{f}_{i}\left(\phi_{i}(\mathbf{p}, \mathbf{q})\right)\right) N_{G} \tilde{}_{G}(\mathbf{p}, \gamma),
$$

where $N^{\sim}{ }_{G}$ does not depend on the $f_{i}$. We claim, and will prove shortly, that the following integrals can be performed.

Definition 5.5. The first integrated function $M_{G, 1}^{\sim}(\mathbf{f}, \gamma)[\mathbf{q}, \boldsymbol{\eta}]$ is the integral

$$
\int_{\mathbb{R}^{(d+1) e(G)}} M^{\sim}(\mathbf{f}, \mathbf{p}, \gamma)[\mathbf{q}, \boldsymbol{\eta}]\left(\prod_{\substack{i=1, \ldots . n \\ \jmath=1, \ldots, s_{\imath}}} \delta\left(\psi_{i, j} \tilde{(}(\mathbf{p}, \mathbf{q})\right)\right) d \mathbf{p}
$$

where the functionals $\tilde{\psi}_{i, j}^{\sim}$ are as follows:

$$
\tilde{\psi}_{i, j}(\mathbf{p}, \mathbf{q})=\tilde{\psi}_{i, j}(G, \mathbf{p}, \boldsymbol{\gamma}, \mathbf{q}, \boldsymbol{\eta})=\psi_{i, j}(G, \mathbf{p}, \gamma)-\sum_{\delta_{G}(k)=X_{\imath, \jmath}} \eta_{k} \mathbf{q}_{k}
$$

$\psi_{i, j}(G, \mathbf{p}, \gamma)$ is the function defined in (3.2.1), and d $\mathbf{p}$ denotes volume integration over $\mathbb{R}^{(d+1) e(G)}$.

Definition 5.6. The second integrated function is the integral

$$
M_{G, 2}^{\sim}(\mathbf{f})[\mathbf{q}, \boldsymbol{\eta}]=\int_{L^{\left|e_{X}(G)\right|}} M_{G, 1}^{\sim}(\mathbf{f}, \boldsymbol{\gamma})[\mathbf{q}, \boldsymbol{\eta}] d \boldsymbol{\gamma}
$$

where $d \gamma$ denotes a product of $\left|e_{X}(G)\right|$ copies of Haar measure on $L$.

Definition 5.7. The partial integral $I_{G}^{\sim}(\mathbf{f})$ is the function

$$
(\mathbf{q}, \boldsymbol{\eta}) \rightarrow \frac{G_{v}^{+} !}{G_{e} !}(-\lambda)^{\left|G_{\mathscr{T}}\right|}(\Delta !)^{-1 / 2} a(G) M_{G, 2}^{\sim}(\mathbf{f})[\mathbf{q}, \boldsymbol{\eta}]
$$

where

$$
G_{v}^{+} !=\prod_{v \in G} d_{+}(v) !
$$

We claim that this function is in $\mathscr{H}_{\Delta}$, for all $\mathbf{f}$ and all pairs $\left(G, \delta_{G}\right)$.

Definition 5.8. For $n>0$, the element $\phi_{M}\left(f_{1}, \ldots, f_{n}\right) \Omega$ is the sum

$$
\sum_{\left(G, \delta_{G}\right) \in \mathscr{S}_{n, p}} I_{G}^{\sim}(\mathbf{f})
$$

which we claim is in $H$. The element $\Omega$ is the constant $1 \in \mathscr{H} b_{0}$. 
The rest of this section is devoted to proving that (5.1.1) does indeed hold for this choice of the vectors $\phi_{M}(\mathbf{f}) \Omega$; which of course implies all the statements about convergence and integrability that we have made so far. The link between definitions (5.3-9) and the $W_{n}$ is as follows.

Definition 5.9. Let $\left(G, \delta_{G}\right) \in \mathscr{G}_{n, p}$ and $\left(G^{\prime}, \delta_{G}^{\prime}\right) \in \mathscr{G}_{m, p}$ be given, such that $\Delta(G)=\Delta\left(G^{\prime}\right)=\Delta$. We define a graph $\Gamma=<G ; G^{\prime}>$, (which we claim is in $\left.\mathscr{G}_{n+m}\right)$, as follows: If $G$ has vertices $\left\{F_{i}, X_{i, j}: i=1, \ldots n, j=1, \ldots . s_{i}\right\}$ and $G^{\prime}$ has vertices $\left\{F_{i}, X_{i, j}: i=1, \ldots . m, j=1, \ldots s_{i}^{\prime}\right\}$ then $\Gamma$ has vertices $\left\{F_{i}, X_{i, j}\right.$ : $\left.i=1, \ldots n+m, j=1, \ldots . s_{i}^{*}\right\}$, where

$$
s_{i}^{*}=\left\{\begin{array}{l}
s_{m+1-i}^{\prime}, \quad i=1, \ldots m \\
s_{i-m}, \quad i=m+1, \ldots m+n
\end{array} .\right.
$$

We define maps $\epsilon: i \rightarrow m+i(i=1, \ldots . n)$ and $\epsilon^{\prime}: i \rightarrow m+1-i(i=1, \ldots . m)$, and we abuse notation slightly by using $\epsilon$ and $\epsilon^{\prime}$ also to denote the maps $G \rightarrow \Gamma$ and $G^{\prime} \rightarrow \Gamma$ such that

$$
\begin{gathered}
\epsilon\left(F_{i}\right)=F_{\epsilon(i)}(i=1, \ldots . n), \\
\epsilon\left(X_{i, j}\right)=X_{\epsilon(i), j}\left(i=1, \ldots . n ; j=1, \ldots . s_{\imath}\right), \\
\epsilon^{\prime}\left(F_{\imath}\right)=F_{\epsilon^{\prime}(i)}(i=1, \ldots . m), \\
\epsilon^{\prime}\left(X_{i, j}\right)=X_{\epsilon^{\prime}(i), 1+s_{\imath}^{\prime}-j}\left(i=1, \ldots . m ; j=1, \ldots . s_{i}^{\prime}\right) .
\end{gathered}
$$

The edges of $\Gamma$ are the union of three sets: (1) The set of all edges $\epsilon(e)=(\epsilon(u), \epsilon(v))$ such that $e=(u, v) \in G$, counted according to multiplicity.

(2) The set of all edges $\epsilon^{\prime}(e)=\left(\epsilon^{\prime}(u), \epsilon^{\prime}(v)\right)$ such that $e=(u, v) \in G^{\prime}$, counted according to multiplicity.

(3) Edges $e_{1}, e_{2}, \ldots, e_{\Delta}$ where each $e_{i}$ connects vertex $\epsilon\left(\delta_{G}(i)\right)$ to vertex $\epsilon^{\prime}\left(\delta_{G}^{\prime}(i)\right)$ $(i=1 . . \Delta)$.

Lemma 5.10. The graph $\Gamma=<G ; G^{\prime}>$ is indeed in $\mathscr{G}_{n+m}$ whenever $\left(G, \delta_{G}\right) \in \mathscr{G}_{n, p}$ and $\left(G^{\prime}, \delta_{G}^{\prime}\right) \in \mathscr{S}_{m, p}$ with $\Delta(G)=\Delta\left(G^{\prime}\right)$. Furthermore, if $M_{G}^{\sim}$ denotes the partial integrand associated with $G$, and $M^{\sim} G^{\prime}$ the partial integrand associated with $G^{\prime}$, then for all sequences $\mathbf{f}, \mathbf{g}, \mathbf{p}, \mathbf{p}^{\prime}, \gamma$ and $\gamma^{\prime}$, we have

$$
\begin{gathered}
M_{G}^{\sim}(\mathbf{f}, \mathbf{p}, \gamma)[\mathbf{q}, \boldsymbol{\eta}] \cdot \overline{M_{G}^{\sim}\left(\mathbf{g}, \mathbf{p}^{\prime}, \gamma^{\prime}\right)[\mathbf{q}, \boldsymbol{\eta}]}= \\
M_{\Gamma}\left(\epsilon(\mathbf{f}, \mathbf{g}), \epsilon\left(\mathbf{p}, \mathbf{p}^{\prime}, \mathbf{q}\right), \epsilon\left(\boldsymbol{\gamma}, \boldsymbol{\gamma}^{\prime}, \boldsymbol{\eta}\right)\right)=M_{\Gamma}\left(\mathbf{f}^{*}, \mathbf{p}^{*}, \gamma^{*}\right)
\end{gathered}
$$

say, where $M_{\Gamma}$ is the graphical integrand of $\Gamma$, the bar denotes complex conjugation, and the maps $\epsilon$ are as follows:

$$
\begin{gathered}
\mathbf{f}^{*}=\epsilon(\mathbf{f}, \mathbf{g}) \text { is the sequence }\left(g_{m}, g_{m-1}, \ldots, g_{1}, f_{1}, f_{2}, f_{3}, \ldots, f_{n}\right), \\
\qquad \mathbf{p}^{*}=\epsilon\left(\mathbf{p}, \mathbf{p}^{\prime}, \mathbf{q}\right) \text { is the sequence }\left(\mathbf{p}_{e}^{*}, e \in e(\Gamma)\right),
\end{gathered}
$$

where

$$
\mathbf{p}_{e}^{*}= \begin{cases}\mathbf{p}_{\alpha}, & \text { if } e=\epsilon(\alpha), \alpha \in e(G) \\ -\mathbf{p}_{\alpha}^{\prime}, & \text { if } e=\epsilon^{\prime}(\alpha), \alpha \in e\left(G^{\prime}\right) \text { of form }\left(F_{i}, X_{i, k}\right) \\ +\mathbf{p}_{\alpha}^{\prime}, & \text { if } e=\epsilon^{\prime}(\alpha), \alpha \in e\left(G^{\prime}\right) \text { is any other edge } \\ \mathbf{q}_{i}, & \text { if } e=e_{i} \text { as in }(5.9 .3) ;\end{cases}
$$


and

$\gamma^{*}=\epsilon\left(\boldsymbol{\gamma}, \gamma^{\prime}, \boldsymbol{\eta}\right)$ is the sequence $\left(\gamma_{e}^{*}, e \in e_{X}(\Gamma)\right)$, where

$$
\gamma_{e}^{*}= \begin{cases}\gamma_{\alpha}, & \text { if } e=\epsilon(\alpha), \alpha \in e_{X}(G) \\ \gamma_{\alpha}^{\prime}, & \text { if } e=\epsilon^{\prime}(\alpha), \alpha \in e_{X}\left(G^{\prime}\right) . \\ \eta_{i}, & \text { if } e=e_{\imath} \text { as in }(5.9 .3)\end{cases}
$$

Proof. In view of (5.9.1), each $s_{i}^{*} \in[0, S]$ so the vertices of $\Gamma$ are in accordance with (2.1). In view of (5.9.2), the vertices of $\Gamma$ are the disjoint union of $\epsilon(G)$ and $\epsilon\left(G^{\prime}\right)$. If $v=\epsilon(u) \in \epsilon(G)$ then by (5.9.3) the edges incident at $v$ are the $\epsilon(e)$ such that $e \in e(G)$ is incident at $u$, together with the edges $e_{i}$ such that $\epsilon\left(\delta_{G}(i)\right)=v$. Therefore the degree

$$
d(v)=d(u)+\left|\delta_{G}^{-1}(u)\right|=d_{+}(u)
$$

using the notation of (5.2.4). Likewise if $v=\epsilon^{\prime}(u)$ for some $u^{\prime} \in G^{\prime}$, we have $d(v)=$ $d_{+}\left(u^{\prime}\right)$. By $(5.2 .1,2)$ we therefore have $d(v)=1$ if $v$ is external, and $d(v) \in[2, N]$ if $v$ is internal; that is, (2.1.1 and 2) hold for all $v \in \Gamma$. If $i \in\{1, \ldots, m\}$ then Band $i$ of $G$ is a graph isomorphic to Band $m+1-i$ of $G^{\prime}$ by the map $\epsilon^{\prime}$, so it is connected; if $i \in\{m+1, \ldots, m+n\}$ Band $i$ is isomorphic to Band $i-m$ of $G$, so it is connected; therefore (2.1.3) holds and $\Gamma$ is in $\mathscr{G}_{n+m}$ as claimed. In order to establish (5.10.1) we follow (3.1.1) and (5.3.1) to rewrite (5.10.1) as:

$$
\prod_{e \in e(G)} M_{G}^{\sim e} \prod_{i=1}^{\Delta} M^{\sim}{ }^{(i)} \prod_{e \in e\left(G^{\prime}\right)} \overline{M_{G^{\prime}}^{\sim e}} \prod_{i=1}^{\Delta} \overline{M_{G^{\prime}}^{(i)}}=\prod_{e \in e(\Gamma)} M_{\Gamma}^{e}
$$

where $M_{G}^{\sim e}$ is $M_{G}^{\sim e}(\mathbf{f}, \mathbf{p}, \gamma)[\mathbf{q}, \boldsymbol{\eta}]$ and $M_{G^{\prime}}^{\sim e}=M_{G^{\prime}}^{\sim e}\left(\mathbf{g}, \mathbf{p}^{\prime}, \gamma^{\prime}\right)[\mathbf{q}, \boldsymbol{\eta}] ; M^{\sim}{ }^{(\imath)}$ is $M^{\sim}{ }_{G}^{(i)}(\mathbf{f}, \mathbf{p}, \boldsymbol{\gamma})[\mathbf{q}, \boldsymbol{\eta}], M^{\sim}{ }_{G^{\prime}}{ }^{(i)}$ is $M^{\sim}{ }_{G^{\prime}}{ }^{(i)}\left(\mathbf{g}, \mathbf{p}^{\prime}, \gamma^{\prime}\right)[\mathbf{q}, \boldsymbol{\eta}]$; and $M_{\Gamma}^{e}$ is

$$
M_{\Gamma}^{e}\left(\epsilon(\mathbf{f}, \mathbf{g}) ; \epsilon\left(\mathbf{p}, \mathbf{p}^{\prime}, \mathbf{q}\right)\right) ; \epsilon\left(\boldsymbol{\gamma}, \boldsymbol{\gamma}^{\prime}, \boldsymbol{\eta}\right)=M_{\Gamma}^{e}\left(\mathbf{f}^{*}, \mathbf{p}^{*}, \gamma^{*}\right)
$$

as in (5.9.3). Because of the three sets (5.9.3) which make up the edges of $\Gamma$, the righthand side of (5.10.8) can be expanded as

$$
\prod_{e \in e(G)} M_{\Gamma}^{\epsilon(e)} \prod_{e \in e\left(G^{\prime}\right)} M_{\Gamma}^{\epsilon^{\prime}(e)} \prod_{i=1}^{\Delta} M_{e_{i}}
$$

Comparing (5.10.8) with (5.10.9) we see that our lemma is proved if we can establish the following three propositions:

(5.10.9) For each $e \in e(G), M_{G}^{\sim e}=M_{\Gamma}^{\epsilon(e)}$.

(5.10.10) For each $e \in e\left(G^{\prime}\right), \overline{M_{G^{\prime}}^{e}}=M_{\Gamma}^{\epsilon^{\prime}(e)}$.

(5.10.11) For each $i=1, \ldots . \Delta, M^{\sim}{ }_{G}^{(i)} \cdot \overline{M^{\sim}{ }^{\prime}{ }^{(i)}}=M_{\Gamma}^{e_{i}}$.

Proof of (5.10.9). Case 1: If $e$ connects $F_{i}$ to $F_{j}$ for some $1 \leq i<j \leq n$ : then by (5.3.3) and (3.1.2), $M_{G}^{\sim e}=\mathrm{H}\left(L_{e}\right) \mu\left(\mathbf{p}_{e}^{2}\right) \widehat{f}_{j}\left(-\mathbf{p}_{e}\right) \widehat{f}_{i}\left(\mathbf{p}_{e}\right)\left|\widehat{\theta}\left(-\gamma_{e} \mathbf{p}_{e}\right)\right|^{2}$ (5.10.12).

Now $\epsilon(e)$ connects vertices $F_{\epsilon(i)}$ and $F_{\epsilon(j)}$ in $\Gamma$, and $\epsilon$ is an increasing function, so by (3.1.2), $M_{G}^{\epsilon(e)}=\mathrm{H}\left(L_{\epsilon(e)}^{*}\right) \mu\left(\left(\mathbf{p}_{\epsilon(e)}^{*}\right)^{2}\right) \widehat{f}_{\epsilon(j)}^{*}\left(-\mathbf{p}_{\epsilon(e)}^{*}\right) \widehat{f}_{\epsilon(i)}^{*}\left(\mathbf{p}_{\epsilon(e)}^{*}\right)\left|\widehat{\theta}\left(-\gamma_{\epsilon(e)}^{*} \mathbf{p}_{\epsilon(e)}^{*}\right)\right|^{2}$ (5.10.13). However, by (5.10.4) $\mathbf{p}_{\epsilon(e)}^{*}=\mathbf{p}_{e}\left(\right.$ and so $\left.L_{\epsilon(e)}^{*}=L_{e}\right)$; by $(5.10 .2) f_{\epsilon(j)}^{*}=f_{j}$ and $f_{\epsilon(i)}^{*}=f_{i}$; and lastly by $(5.10 .4), \gamma_{\epsilon(e)}^{*}=\gamma_{e}$. So, (5.10.12) and (5.10.13) are identical as required. 
Case 2: If $e$ connects $F_{\imath}$ to $X_{j, k}(j \neq i)$, or if $e$ connects $X_{i, j}$ to $X_{k, l}$, then the equality of $M_{G}^{\sim e}$ and $M_{\Gamma}^{\epsilon(e)}$ is established using (3.1) and (5.3) by methods closely analogous to those of Case 1 (we will investigate these cases more closely when proving (5.10.10), when some interesting changes of sign occur).

Case 3: If $e$ connects $F_{i}$ to $X_{i, k}$ then by (5.3.2), $M_{G}^{\sim e}=\mu\left(\mathbf{p}_{e}^{2}\right) \widehat{f_{i}}(\psi) \cdot\left|\widehat{\theta}\left(\mathbf{p}_{e}\right)\right|^{2}$ (5.10.14) where

$$
\psi=\phi_{\imath}(\mathbf{p})-\sum_{\delta_{G}(j) \in B_{i}(G)} \mathbf{q}_{j},
$$

$\phi_{i}(\mathbf{p})$ is the flow within graph $G$ as in (3.1.5), and $B_{i}$ is the ith band of $G$. On the other hand, $\epsilon(e)$ connects $F_{\epsilon(i)}$ to $X_{\epsilon(i), k}$ so by (3.1.5),

$$
M_{\Gamma}^{\epsilon(e)}=\mu\left(\left(\mathbf{p}_{\epsilon(e)}^{*}\right)^{2}\right) \widehat{f}_{\epsilon(i)}^{*}\left(\phi_{\epsilon(i)}^{*}\left(\mathbf{p}^{*}\right)\right)\left|\widehat{\theta}\left(\mathbf{p}_{\epsilon(e)}^{*}\right)\right|^{2},
$$

where $\phi_{\epsilon(i)}^{*}$ denotes the flow within the graph $\Gamma$ as in (3.1.5). As in Case 1 we have $f_{\epsilon(i)}^{*}=f_{i}$ and $\mathbf{p}_{\epsilon(e)}^{*}=\mathbf{p}_{e}$; so all that remains to be proved is that

$$
\phi_{\epsilon(\imath)}^{*}\left(\mathbf{p}^{*}\right)=\phi_{i}(\mathbf{p})-\sum_{\delta_{G}(j) \in B_{i}(G)} \mathbf{q}_{j} .
$$

Now by (3.1.5),

$$
\phi_{\epsilon(\imath)}^{*}\left(\mathbf{p}^{*}\right)=\sum_{e^{\prime} \backslash \epsilon(\imath) \text { in } \Gamma} \mathbf{p}_{e^{\prime}}^{*}-\sum_{e^{\prime} / \epsilon(\imath) \text { in } \Gamma} \mathbf{p}_{e^{\prime}}^{*}
$$

and by (5.9.3) the edges $e \searrow \epsilon(i)$ in $\Gamma$ are precisely those $\epsilon\left(e^{\prime \prime}\right)$ with $e^{\prime \prime} \searrow i$ in $G$. Moreover the $e^{\prime} \nearrow \epsilon(i)$ in $\Gamma$ are the union of those $\epsilon\left(e^{\prime \prime}\right)$ with $e^{\prime \prime} \nearrow i$ in $G$, together with all $e_{\jmath}$ such that the special edge $e_{\jmath}$ of (5.9.3) Case 3 enters band $\epsilon(i)$. Hence,

$$
\phi_{\epsilon(i)}^{*}\left(\mathbf{p}^{*}\right)=\sum_{\substack{e^{\prime \prime}>i \\ e^{\prime \prime} \in G}} \mathbf{p}_{\epsilon(i)}^{*}-\sum_{\substack{e^{\prime \prime} / i \\ e^{\prime \prime} \in G}} \mathbf{p}_{\epsilon(i)}^{*}-\sum_{\delta_{G}(j) \in B_{i}(G)} \mathbf{p}_{e_{\jmath}}^{*},
$$

hence we obtain (5.10.16), and so we prove our result in Case 3. Thus (5.10.9) is proved.

Proof of (5.10.10).

Case 1: If $e$ connects $F_{i}$ to $F_{j}$ for some $1 \leq i<j \leq m$, then by (5.3.3) and (3.1.2),

$$
M_{G^{\prime}}^{\sim}=\mathrm{H}\left(L_{e}^{\prime}\right) \mu\left(\left(\mathbf{p}_{e}^{\prime}\right)^{2}\right) \widehat{g}_{j}\left(-\mathbf{p}_{e}^{\prime}\right) \widehat{g}_{\imath}\left(\mathbf{p}_{e}^{\prime}\right)\left|\widehat{\theta}^{\prime}\left(\gamma_{e}^{\prime} \mathbf{p}_{e}^{\prime}\right)\right|^{2} .
$$

Now $\epsilon^{\prime}(e)$ connects vertices $F_{\epsilon^{\prime}(i)}$ and $F_{\epsilon^{\prime}(j)}$ in $\Gamma$, and $\epsilon^{\prime}$ is a decreasing function, so by (3.1.2), since $\epsilon^{\prime}(j)<\epsilon^{\prime}(i)$,

$$
M_{\Gamma}^{\epsilon^{\prime}(e)}=\mathrm{H}\left(L_{\epsilon^{\prime}(e)}^{*}\right) \mu\left(\left(\mathbf{p}_{\epsilon^{\prime}(e)}^{*}\right)^{2}\right) \widehat{f}_{\epsilon^{\prime}(i)}^{*}\left(-\mathbf{p}_{\epsilon^{\prime}(e)}^{*}\right) \widehat{f}_{\epsilon^{\prime}(j)}^{*}\left(\mathbf{p}_{\epsilon^{\prime}(e)}^{*}\right)\left|\widehat{\theta}\left(\gamma_{\epsilon^{\prime}(e)}^{*} \mathbf{p}_{\epsilon^{\prime}(e)}^{*}\right)\right|^{2} .
$$

Now by (5.10.2-6), $\mathbf{p}_{\epsilon^{\prime}(e)}^{*}=\mathbf{p}_{e}^{\prime}, f_{\epsilon^{\prime}(i)}^{*}=g_{i}, f_{\epsilon^{\prime}(j)}^{*}=g_{j}$ and $\gamma_{\epsilon^{\prime}(e)}^{*}=\gamma_{e}^{\prime}$. So, (5.10.18) is equal to

$$
\mathrm{H}\left(L_{e}^{\prime}\right) \mu\left(\left(\mathbf{p}_{e}^{\prime}\right)^{2}\right) \widehat{g}_{\imath}\left(-\mathbf{p}_{e}^{\prime}\right) \widehat{g}_{j}\left(\mathbf{p}_{e}^{\prime}\right)\left|\widehat{\theta}\left(\gamma_{e}^{\prime} \mathbf{p}_{e}^{\prime}\right)\right|^{2} .
$$

Since $\mu, g_{i}$ and $g_{j}$ are all real valued functions, this is indeed the complex conjugate of (5.10.17).

Case 2: If $e$ connects $F_{i}$ to $X_{j, k}(j \neq i)$, then by $(5.3 .3),(3.1 .3,4)$ we have 


$$
M_{G^{\prime}}^{\sim e}=\left\{\begin{array}{ll}
\mathrm{H}\left(L_{e}^{\prime}\right) \mu\left(\left(\mathbf{p}_{e}^{\prime}\right)^{2}\right) \widehat{g}_{i}\left(\mathbf{p}_{e}^{\prime}\right)\left|\widehat{\theta}^{\prime}\left(\gamma_{e}^{\prime} \mathbf{p}_{e}^{\prime}\right)\right|^{2}, & i<j \\
\mathrm{H}\left(L_{e}^{\prime}\right) \mu\left(\left(\mathbf{p}_{e}^{\prime}\right)^{2}\right) \widehat{g}_{i}\left(-\mathbf{p}_{e}^{\prime}\right)\left|\widehat{\theta}^{\prime}\left(\gamma_{e}^{\prime} \mathbf{p}_{e}^{\prime}\right)\right|^{2}, & i>j
\end{array} .\right.
$$

Now the sign of $\epsilon^{\prime}(i)-\epsilon^{\prime}(j)$ is opposite to that of $i-j$ by (5.9.1); so since $\epsilon^{\prime}(e)$ connects $F_{\epsilon^{\prime}(i)}$ to $X_{\epsilon^{\prime}(j), 1+s_{i}^{\prime}-j}$, by $(3.1 .3,4)$ we have

$$
\begin{aligned}
& M_{\Gamma}^{\epsilon^{\prime}(e)}= \begin{cases}\left.\mathrm{H}\left(L_{\epsilon^{\prime}(e)}^{*}\right) \mu\left(\left(\mathbf{p}_{\epsilon^{\prime}(e)}^{*}\right)\right)^{2}\right) \widehat{f}_{\epsilon^{\prime}(i)}^{*}\left(-\mathbf{p}_{\epsilon^{\prime}(e)}^{*}\right)\left|\widehat{\theta}\left(\gamma_{\epsilon^{\prime}(e)}^{*} \mathbf{p}_{\epsilon^{\prime}(e)}^{*}\right)\right|^{2}, & i<j, \\
\mathrm{H}\left(L_{\epsilon^{\prime}(e)}^{*}\right) \mu\left(\left(\mathbf{p}_{\epsilon^{\prime}(e)}^{*}\right)^{2}\right) \widehat{f}_{\epsilon^{\prime}(e)}^{*}\left(\mathbf{p}_{\epsilon^{\prime}(e)}^{*}\right)\left|\hat{\theta}\left(\gamma_{\epsilon^{\prime}(e)}^{*} \mathbf{p}_{\epsilon^{\prime}(e)}^{*}\right)\right|^{2}, & i>j\end{cases} \\
& = \begin{cases}\mathrm{H}\left(L_{e}^{\prime}\right) \mu\left(\left(\mathbf{p}_{e}^{\prime}\right)^{2}\right) \widehat{g}_{i}\left(-\mathbf{p}_{e}^{\prime}\right)\left|\widehat{\theta}\left(\gamma_{e}^{\prime} \mathbf{p}_{e}^{\prime}\right)\right|^{2}, & i<j, \\
\mathrm{H}\left(L_{e}^{\prime}\right) \mu\left(\left(\mathbf{p}_{e}^{\prime}\right)^{2}\right) \widehat{g}_{i}\left(\mathbf{p}_{e}^{\prime}\right)\left|\widehat{\theta}\left(\gamma_{e}^{\prime} \mathbf{p}_{e}^{\prime}\right)\right|^{2}, & i>j\end{cases}
\end{aligned}
$$

by the usual substitutions of $(5.10 .3,4)$. Since $g_{i}$ is real-valued, this is indeed the complex conjugate of (5.10.19).

Case 3: If $e$ connects $F_{i}$ to $X_{\imath, k}$, then arguing as in Case 3 of (5.10.9), we find that

$$
\overline{M_{G^{\prime}}^{\sim e}}=\mu\left(\left(\mathbf{p}_{e}^{\prime}\right)^{2}\right) \widehat{g}_{i}(-\psi) \cdot\left|\widehat{\theta}\left(\mathbf{p}_{e}^{\prime}\right)\right|^{2},
$$

where $\psi=\phi_{i}^{\prime}\left(\mathbf{p}^{\prime}\right)-\sum_{\delta_{G}^{\prime}(\jmath) \in B_{i}\left(G^{\prime}\right)} \mathbf{q}_{j}$, and $\phi_{i}^{\prime}\left(\mathbf{p}^{\prime}\right)$ is the flow within graph $G^{\prime}$ as in (3.1.5), and $B_{i}$ is the ith band of $G^{\prime}$. On the other hand, $\epsilon(e)$ connects $F_{\epsilon(\imath)}$ to $X_{\epsilon(i), k}$ so by (3.1.5),

$$
M_{\Gamma}^{\epsilon^{\prime}(e)}=\mu\left(\left(\mathbf{p}_{\epsilon^{\prime}(e)}^{*}\right)^{2}\right) \widehat{g}_{\epsilon^{\prime}(i)}^{*}\left(\phi_{\epsilon^{\prime}(\imath)}^{*}\left(\mathbf{p}^{*}\right)\right)\left|\widehat{\theta}\left(\mathbf{p}_{\epsilon^{\prime}(e)}^{*}\right)\right|^{2},
$$

where $\phi_{\epsilon(i)}^{*}$ denotes the flow within the graph $\Gamma$ as in (3.1.5). As in Case 1 we have $f_{\epsilon(i)}^{*}=g_{i}$. By the special case of (5.10.3) we have $\mathbf{p}_{\epsilon^{\prime}(e)}^{*}=-\mathbf{p}_{e}$. Note that the change of sign doesn't matter- $\theta$ is a real valued function so $|\widehat{\theta}|$ is an even function. So, all that remains to be proved is that

$$
\phi_{\epsilon^{\prime}(i)}^{*}\left(\mathbf{p}^{*}\right)=-\phi_{i}^{\prime}\left(\mathbf{p}^{\prime}\right)+\sum_{\delta_{G^{\prime}}(j) \in B_{\imath}\left(G^{\prime}\right)} \mathbf{q}_{j} .
$$

Now by (3.1.5),

$$
\phi_{\epsilon^{\prime}(i)}^{*}\left(\mathbf{p}^{*}\right)=\sum_{e^{\prime} \searrow \epsilon^{\prime}(i) \text { in } \Gamma} \mathbf{p}_{e^{\prime}}^{*}-\sum_{e^{\prime} / \epsilon^{\prime}(\imath) \text { in } \Gamma} \mathbf{p}_{e^{\prime}}^{*}
$$

and by (5.9.3) the edges $e \nearrow \epsilon^{\prime}(i)$ in $\Gamma$ are precisely those $\epsilon^{\prime}\left(e^{\prime \prime}\right)$ with $e^{\prime \prime} \searrow i$ in $G$. Moreover the $e^{\prime} \searrow \epsilon^{\prime}(i)$ in $\Gamma$ are the union of those $\epsilon\left(e^{\prime \prime}\right)$ with $e^{\prime \prime} \nearrow i$ in $G$, together with all $e_{j}$ such that the special edge $e_{j}$ of (5.9.3) Case 3 enters band $\epsilon^{\prime}(i)$. Note now that for all edges $e^{\prime \prime}$ that we have mentioned, we have $\mathbf{p}_{\epsilon^{\prime}\left(e^{\prime \prime}\right)}^{*}=\mathbf{p}_{e^{\prime \prime}}^{\prime}$; because the special case of (5.10.3) (where the sign changes and $\mathbf{p}_{\epsilon^{\prime}(f)}^{*}=-\mathbf{p}_{f}^{\prime}$ ) involves an edge $f=\left(F_{l}, X_{l, k}\right)$ of $G^{\prime}$ which is not in $e_{X}\left(G^{\prime}\right)$ because it is internal to Band $l$ of $G^{\prime}$. Hence, we find that

$$
\begin{gathered}
\phi_{\epsilon^{\prime}(i)}^{*}\left(\mathbf{p}^{*}\right)=\sum_{\substack{e^{\prime \prime} \nearrow_{i} \\
e^{\prime \prime} \in G}} \mathbf{p}_{\epsilon^{\prime}\left(e^{\prime \prime}\right)}^{*}-\sum_{\substack{e^{\prime \prime \backslash j i} \\
e^{\prime \prime} \in G}} \mathbf{p}_{\epsilon^{\prime}\left(e^{\prime \prime}\right)}^{*}+\sum_{\delta_{G^{\prime}}(j) \in B_{\imath}\left(G^{\prime}\right)} \mathbf{p}_{e_{j}}^{*} \\
=-\phi_{i}^{\prime}\left(\mathbf{p}^{\prime}\right)+\sum_{\delta_{G^{\prime}}(j) \in B_{i}\left(G^{\prime}\right)} \mathbf{q}_{j}
\end{gathered}
$$


and Case 3 is established.

Case 4: If $e$ connects $X_{i, j}$ to $X_{k, l}$ with $i<k$ then (5.3.3), (3.1.6) give us

$$
M_{G^{\prime}}^{\sim e}=\mathrm{H}\left(L_{e}^{\prime}\right) \mu\left(\left(\mathbf{p}_{e}^{\prime}\right)^{2}\right)\left|\widehat{\theta}^{\prime}\left(\gamma_{e}^{\prime} \mathbf{p}_{e}^{\prime}\right)\right|^{2},
$$

which is a real number; and (3.1.6) gives us

$$
M_{\Gamma}^{\epsilon^{\prime}(e)}=\mathrm{H}\left(L_{\epsilon^{\prime}(e)}^{*}\right) \mu\left(\left(\mathbf{p}_{\epsilon^{\prime}(e)}^{*}\right)^{2}\right)\left|\widehat{\theta}\left(\gamma_{\epsilon^{\prime}(e)}^{*} \mathbf{p}_{\epsilon^{\prime}(e)}^{*}\right)\right|^{2},
$$

which by $(5.10 .3,4)$ is the same real number.

Case 5: If $e$ connects $X_{i, j}$ to $X_{i, l}$ with $j<l$ then (5.3.3), (3.1.7) give us

$$
M_{G^{\prime}}^{\sim e}=\mathrm{H}\left(L_{e}^{\prime}\right) \mu\left(\left(\mathbf{p}_{e}^{\prime}\right)^{2}\right)\left|\widehat{\theta}\left(\mathbf{p}_{e}^{\prime}\right)\right|^{2},
$$

and (3.1.7) gives us

$$
M_{\Gamma}^{\epsilon^{\prime}(e)}=\mathrm{H}\left(L_{\epsilon^{\prime}(e)}^{*}\right) \mu\left(\left(\mathbf{p}_{\epsilon^{\prime}(e)}^{*}\right)^{2}\right)\left|\widehat{\theta}\left(\mathbf{p}_{\epsilon^{\prime}(e)}^{*}\right)\right|^{2},
$$

which by $(5.10 .3,4)$ is the same real number. This concludes the proof of $(5.10 .10)$. Proof of (5.10.11).

Case 1: $\delta_{G}(i)$ is an external vertex $F_{j}$ of $G$, and $\delta_{G^{\prime}}(i)$ is an internal vertex $X_{k, l}$ of $G^{\prime}$. Then by (5.3.3) and (5.3.4), $M^{\sim}{ }_{G}{ }^{(i)}=\mathrm{H}\left(L_{\imath}\right) \sqrt{\mu\left(\mathbf{q}_{i}^{2}\right)} \cdot \widehat{f}_{j}\left(-\mathbf{q}_{i}\right) \cdot \widehat{\theta}\left(-\eta_{i} \mathbf{q}_{i}\right)$ and $M^{\sim}{ }_{G^{\prime}}{ }^{(i)}=\mathrm{H}\left(L_{i}\right) \sqrt{\mu\left(\mathbf{q}_{i}^{2}\right)} \cdot \widehat{\theta}\left(-\eta_{i} \mathbf{q}_{i}\right)$ so the product $M^{\sim}{ }_{G}{ }^{(\imath)} \overline{M^{\sim}{ }_{G^{\prime}}{ }^{(\imath)}}$ is equal to

$$
\mathrm{H}\left(L_{i}\right) \mu\left(\mathbf{q}_{i}^{2}\right) \cdot\left|\widehat{\theta}\left(-\eta_{i} \mathbf{q}_{i}\right)\right|^{2} .
$$

Now the edge $e_{i}$ connects $F_{\epsilon(j)}$ to $X_{\epsilon^{\prime}(k), l}$; certainly $\epsilon(j) \in(m, m+n]$ is greater than $\epsilon^{\prime}(k) \in[1, m]$ so by (3.1.4), $M_{\Gamma}^{e_{2}}=\mathrm{H}\left(L_{e_{2}}^{*}\right) \mu\left(\left(\mathbf{p}_{e_{2}}^{*}\right)^{2}\right) \widehat{f}_{\epsilon(j)}^{*}\left(-\mathbf{p}_{e_{2}}^{*}\right)\left|\widehat{\theta}\left(-\gamma_{e_{i}}^{*} \mathbf{p}_{e_{i}}^{*}\right)\right|^{2}$ and the usual substitutions obtained from $(5.10 .2-6)$ tell us that this is indeed equal to (5.10.19).

Case 2: If the pair $\left(\delta_{G}(i), \delta_{G^{\prime}}(i)\right)$ consists either of two internal vertices, or two external vertices, or a pair $\left(X_{k, l}, F_{j}\right)$ the opposite way round to Case 1 ; then similar calculations give the result. This concludes the proof of Lemma 5.10.

Corollary 5.11. Let $\left(G, \delta_{G}\right) \in \mathscr{G}_{n, p}$ and $\left(G^{\prime}, \delta_{G^{\prime}}\right) \in \mathscr{G}_{m, p}$ with $\Delta(G)=\Delta\left(G^{\prime}\right)=\Delta$. Let sequences $\mathbf{f}, \mathbf{g}, \gamma, \gamma^{\prime}, \mathbf{q}$ and $\boldsymbol{\eta}$ be given as before. Then the first partial moments $M_{G, 1}^{\sim}(\mathbf{f}, \gamma)$ and $M_{G^{\prime}, 1}^{\sim}\left(\mathbf{g}, \gamma^{\prime}\right)$ exist for almost every value of $\mathbf{q} \in \mathbb{R}^{\Delta}$, and

$$
\int_{\mathbb{B}^{(d+1) \Delta}} M_{G, 1}^{\sim}(\mathbf{f}, \boldsymbol{\gamma})[\mathbf{q}, \boldsymbol{\eta}] \cdot \overline{M_{G^{\prime}, 1}\left(\mathbf{g}, \boldsymbol{\gamma}^{\prime}\right)[\mathbf{q}, \boldsymbol{\eta}]} d \mathbf{q}=M_{\Gamma, 1}\left(\mathbf{f}^{*}, \boldsymbol{\gamma}^{*}\right),
$$

where $\Gamma$ is the graph $\left\langle G ; G^{\prime}>\right.$ defined in (5.9), $M_{\Gamma, 1}$ is the first moment as in (3.2), and $\mathbf{f}^{*}, \gamma^{*}$ are as in (5.10.2-6).

Proof . To establish existence of $M^{\sim} G_{1,1}$, let us momentarily substitute $G^{\prime}=G$ in (5.10.1), so that we are temporarily dealing with a graph $\Gamma=<G ; G>$. Then, let us multiply (5.10.1) by some delta-functions and integrate $d \mathbf{p}^{*}=d \mathbf{p} d \mathbf{p}^{\prime} d \mathbf{q}$ so as to obtain the first integrated moment $M_{<G ; G>, 1}\left(\mathbf{f}^{*}, \gamma^{*}\right)$ of the graph $<G ; G>$, just as in (3.2). (here $\mathbf{f}^{*}$ temporarily denotes the sequence $f_{n}, \ldots, f_{1}, f_{1}, \ldots, f_{n}$ ). We claim that the appropriate delta-functions are none other than the functions 
$\delta\left(\psi_{i, j}(G, \mathbf{p}, \boldsymbol{\gamma}, \mathbf{q}, \boldsymbol{\eta})\right)$ and $\delta\left(\psi_{i, j}^{\sim}\left(G, \mathbf{p}^{\prime}, \boldsymbol{\gamma}^{\prime}, \mathbf{q}, \boldsymbol{\eta}\right)\right)$, where the $\psi^{\sim}{ }_{i, j}$ are as in (5.5), and all values $i=1, \ldots n, j=1, \ldots . . s_{\imath}$ are used.

Proof of Claim. We are, by (3.2), trying to identify the product of all functions $\delta\left(\psi_{i, j}\left(<G ; G>, \mathbf{p}^{*}, \gamma^{*}\right)\right)$ for $X_{i, j}$ an internal vertex of $\langle G ; G\rangle$.

The graph $<G ; G>$ consists of two copies $\epsilon(G)$ and $\epsilon^{\prime}(G)$ of the graph $\mathrm{G}$, together with certain connecting edges joining vertices $\epsilon\left(\delta_{G}(i)\right)$ to $\epsilon^{\prime}\left(\delta_{G}(i)\right)$.

Accordingly, each internal vertex of $\langle G ; G\rangle$ is either $\epsilon\left(X_{i, j}\right)=X_{\epsilon(i), j}$ or $\epsilon^{\prime}\left(X_{i, j}\right)=X_{\epsilon^{\prime}(i), 1+s_{i}-j}$; for some internal vertex $X_{i, j}$ of $G$.

Case 1: Consider an internal vertex $X_{\epsilon(\imath), j}$. The flow $\psi_{\epsilon(i), j}\left(<G ; G>, \mathbf{p}^{*}, \gamma^{*}\right)$ may be calculated from (3.2.1) as the sum of all $\gamma_{f}^{*} \mathbf{p}_{f}^{*}$ such that edge $f$ connects $X_{\epsilon(i), j}$ to a vertex "above" it in the sense of (3.2), minus the sum of all the $\gamma_{f}^{*} \mathbf{p}_{f}^{*}$ such that edge $f$ connects $X_{\epsilon(i), j}$ to a vertex "below" it. Now all edges of form $f=$ $e_{i}=\left(\epsilon^{\prime}\left(\delta_{G}(i)\right), \epsilon\left(\delta_{G}(i)\right)\right)(i=1, \ldots . \Delta)$ as in (5.9(3)) have $\epsilon^{\prime}\left(\delta_{G}(i)\right)$ "below" $\epsilon\left(\delta_{G}(i)\right)$, because the former is in one of Bands 1 to $n$ and the latter in one of Bands $n+1$ to $2 n$ of $\langle G ; G\rangle$. Also, if $f=\epsilon(e)$ for some edge $e$, then since $\epsilon$ is an "increasing" map with respect to the ordering of (3.2) on $G$ and $\langle G ; G\rangle$, we find that $\gamma_{f}^{*} \mathbf{p}_{f}^{*}$ is counted towards $\psi_{\epsilon(i), j}\left(<G ; G>, \mathbf{p}^{*}, \gamma^{*}\right)$ with the same sign as $\gamma_{e} \mathbf{p}_{e}$ in $\psi_{i, j}(G, \mathbf{p}, \gamma)$. Hence, if we make our standard set of substitutions as in (5.10.1-3), we have

$$
\psi_{\epsilon(i), \jmath}\left(<G ; G>, \mathbf{p}^{*}, \gamma^{*}\right)=\psi_{i, j}(G, \mathbf{p}, \gamma)-\sum_{\delta_{G}(k)=X_{\imath, \jmath}} \eta_{k} \mathbf{q}_{k}=\tilde{\psi}_{i, \jmath}(G, \mathbf{p}, \boldsymbol{\gamma}, \mathbf{q}, \boldsymbol{\eta}) .
$$

Well, that gets us half of the delta-functions required by our claim.

Case 2: Consider an internal vertex $X_{\epsilon^{\prime}(i), 1+s_{i}-j}=\epsilon^{\prime}\left(X_{i, j}\right)$. Then the flow

$$
\psi_{\epsilon^{\prime}(i), 1+s_{i}-j}\left(<G ; G>, \mathbf{p}^{*}, \gamma^{*}\right)
$$

counts positively each $\gamma_{f}^{*} \mathbf{p}_{f}^{*}$ such that the edge $f=e_{i}=\left(\epsilon^{\prime}\left(\delta_{G}(i)\right), \epsilon\left(\delta_{G}(i)\right)\right)$. It also counts positively each $\gamma_{f}^{*} \mathbf{p}_{f}^{*}$ such that the edge $f=\epsilon^{\prime}(e)$ connects $\epsilon^{\prime}\left(X_{i, j}\right)$ to a vertex "above" it in $\langle G ; G>$, and counts the same quantity negatively for each edge $f=\epsilon^{\prime}(e)$ connecting $\epsilon^{\prime}\left(X_{i, j}\right)$ to a vertex "below" it in $\langle G ; G\rangle$. Now the map $\epsilon^{\prime}: G \rightarrow<G: G>$ reverses the ordering of all pairs of vertices involved in edges of $G$, with the sole exception of an edge of form $\left(F_{k}, X_{k, l}\right)$. In this case only, the ordering remains the same (with $X_{i, j}$ deemed to be "above" $F_{i}$ ). But in this case, only, we find that (5.10.3) gives $\mathbf{p}_{f}^{*}=-\mathbf{p}_{e}^{\prime}$ (whereas in all other cases, $\mathbf{p}_{f}^{*}=+\mathbf{p}_{e}^{\prime}$ ). Hence,

$$
\begin{aligned}
\psi_{\epsilon^{\prime}(i), 1+s_{\imath}^{\prime}-\jmath}(<G ; G & \left.>, \mathbf{p}^{*}, \boldsymbol{\gamma}^{*}\right)=-\psi_{i, j}\left(G, \mathbf{p}^{\prime}, \boldsymbol{\gamma}^{\prime}\right)+\sum_{\delta_{G}(k)=X_{\imath, \jmath}} \eta_{k} \mathbf{q}_{k} \\
& =-\psi^{\sim}{ }_{i, j}\left(G, \mathbf{p}^{\prime}, \boldsymbol{\gamma}^{\prime}, \mathbf{q}, \boldsymbol{\eta}\right) .
\end{aligned}
$$

Accordingly - $\delta$ being an even function! - we have

$$
\delta\left(\psi_{\epsilon^{\prime}(i), 1+s_{\imath}-j}\left(<G ; G>, \mathbf{p}^{*}, \gamma^{*}\right)\right)=\delta\left(\psi_{i, j}^{\sim}\left(G, \mathbf{p}^{\prime}, \gamma^{\prime}, \mathbf{q}, \boldsymbol{\eta}\right)\right) .
$$

This gives us our second set of delta-functions and proves our Claim.

Let us therefore make our substitution $G^{\prime}=G$ in (5.10.1), multiply by our well known set of delta-functions, and integrate. By (4.3) our integral is (absolutely) convergent, so we have 


$$
\begin{aligned}
& \int_{\mathbb{R}^{(d+1) e(G)}} d \mathbf{p} \int_{\mathbb{B}^{(d+1) e(G)}} d \mathbf{p}^{\prime} \int_{\mathbb{R}^{(d+1) \Delta}} d \mathbf{q} M^{\sim}{ }_{G}(\mathbf{f}, \mathbf{p}, \gamma)[\mathbf{q}, \boldsymbol{\eta}] \overline{M_{G}^{\sim}\left(\mathbf{f}, \mathbf{p}^{\prime}, \boldsymbol{\gamma}^{\prime}\right)[\mathbf{q}, \boldsymbol{\eta}]} \times
\end{aligned}
$$

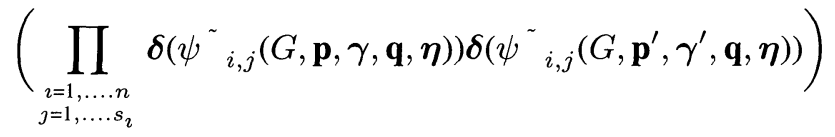

$$
\begin{aligned}
& =\int_{\mathbb{R}^{(d+1) \Delta}}\left|M_{G, 1}^{\sim}(\mathbf{f}, \gamma)[\mathbf{q}, \boldsymbol{\eta}]\right|^{2} d \mathbf{q}=M_{<G ; G>, 1}\left(\mathbf{f}^{*}, \gamma^{*}\right)<\infty .
\end{aligned}
$$

Likewise, $M^{\sim} G^{\prime}, 1\left(\mathbf{g}, \gamma^{\prime}\right)$ is almost surely finite and square-integrable with respect to q. Now, we may take (5.10.1) itself, multiply it by the product of the appropriate delta functions (namely, all functions $\delta\left(\psi_{i, j}^{\sim}(G, \mathbf{p}, \boldsymbol{\gamma}, \mathbf{q}, \boldsymbol{\eta})\right)$ and $\delta\left(\psi_{i, j}{ }_{i, j}\left(G^{\prime}, \mathbf{p}^{\prime}, \gamma^{\prime}, \mathbf{q}\right.\right.$, $\boldsymbol{\eta}))$ ), integrate $d \mathbf{p} d \mathbf{p}^{\prime} d \mathbf{q}$, and so obtain (5.11.1). Thus our corollary is proved.

Corollary 5.12. The second partial moments $M_{G, 2}^{\sim}(\mathbf{f})[\mathbf{q}, \boldsymbol{\eta}]$ and $M_{G^{\prime}, 2}^{\sim}(\mathbf{g})[\mathbf{q}, \boldsymbol{\eta}]$ exist for almost every pair $(\mathbf{q}, \boldsymbol{\eta}) \in U^{\Delta}$ (where "almost every" is in the sense of the measure $(d \mathbf{q} \times d \eta)^{\Delta}$ of (5.1)). Moreover, the integral

$$
\int_{U^{\Delta}} M_{G, 2}^{\sim}(\mathbf{f})[\mathbf{q}, \boldsymbol{\eta}] \overline{M_{G^{\prime}, 2}(\mathbf{g})[\mathbf{q}, \boldsymbol{\eta}]} d \mathbf{q} d \boldsymbol{\eta}=<M_{G, 2}^{\sim}(\mathbf{f}), M_{G^{\prime}, 2}^{\sim}(\mathbf{g})>=M_{\Gamma, 2}\left(\mathbf{f}^{*}\right),
$$

where the inner product is that of $\mathscr{H}_{\Delta} . M_{G, 2}^{\sim}(\mathbf{f})$ is in $\mathscr{H}_{\Delta}$.

Proof. The fact that the integral over $U^{\Delta}$ is the inner product in $\mathscr{H}_{\Delta}$ is Definition 5.1. The fact that it is equal to $M_{\Gamma, 2}\left(\mathbf{f}^{*}\right)$ follows by integrating (5.11.1) with respect to $\gamma^{*}$, recalling that $\gamma^{*}$ is essentially the union of the three sequences $\gamma, \gamma^{\prime}$ and $\eta$ (see (5.10.6)). The details are rather simpler than those of (5.11) (no delta-functions to worry about!) so we omit them.

Lemma 5.13. Let $n>0$ and $m>0$ be fixed. For each graph $\Gamma \in \mathscr{G}_{n+m}$ there is a unique pair of graphs $\left(G, G^{\prime}\right)$ such that there is any pair $\left(\delta_{G}, \delta_{G^{\prime}}\right)$ with $\left(G, \delta_{G}\right) \in$ $\mathscr{G}_{n, p} ;\left(G^{\prime}, \delta_{G^{\prime}}\right) \in \mathscr{G}_{m, p}$ and $<G ; G^{\prime}>=\Gamma$.

Moreover, the total number of such pairs is equal to

$$
\Delta ! \cdot \frac{G_{e} ! G_{e}^{\prime} !}{\Gamma_{e} !},
$$

where $\Delta=\Delta(G)=\Delta\left(G^{\prime}\right)$ is the number of edges of $\Gamma$ that cross the "great divide" between Bands $1 \ldots m$ and Bands $m+1 \ldots m+n$.

Proof. The graph $G$ must be isomorphic to the subgraph of $\Gamma$ consisting of Bands $m+1, \ldots, m+n$; and it must be isomorphic via the map $\epsilon: G \rightarrow \Gamma$ of (5.9). So there is only one such $G$, likewise there is only one $G^{\prime}$; but given $\Gamma$ we can construct $G$ and $G^{\prime}$ from the two subgraphs, and a suitable pair $\left(\delta_{G}, \delta_{G^{\prime}}\right)$ is then as follows: Let the edges of $\Gamma$ which cross from one of Bands $1 \ldots m$ to one of Bands $m+1 \ldots m+n$ be $e_{1}^{*}, \ldots, e_{\Delta}^{*}$ in some order, and let us say that $e_{i}^{*}$ connects vertex $\epsilon\left(u_{i}\right)$ to $\epsilon^{\prime}\left(v_{i}\right)$, where $\epsilon$ and $\epsilon^{\prime}$ are as in (5.9). If we then define $\delta_{G}(i)=u_{i}$ and $\delta_{G^{\prime}}(i)=v_{i}$, then the edge $e_{i}$ of $\left\langle G ; G^{\prime}>\right.$ given by (5.9.3) is none other than $\left(\epsilon\left(u_{i}\right), \epsilon^{\prime}\left(v_{i}\right)\right)=e_{i}^{*}$. So, $<G ; G^{\prime}>=\Gamma$ with this choice of $\delta_{G}, \delta_{G^{\prime}}$ because the "crossing edges" $e_{1}^{*}, \ldots, e_{\Delta}^{*}$ are faithfully reproduced according to multiplicity. 
Now any other pair $\left(\zeta_{G}, \zeta_{G^{\prime}}\right)$ which replaces $\left(\delta_{G}, \delta_{G^{\prime}}\right)$ and gives rise to the graph, will have to do so by having the same value of $\Delta$, and its special edges $e_{i}$ will be $e_{\pi(\imath)}^{*}$ for some permutation $\pi$ on $\{1, \ldots, \Delta\}$ (so, $\Delta$ ! possibilities); we will then have

$$
\left(\zeta_{G}, \zeta_{G^{\prime}}\right)=\left(\delta_{G} \circ \pi, \delta_{G^{\prime}} \circ \pi\right)
$$

However, not all permutations $\pi$ give rise to distinct pairs $\left(\zeta_{G}, \zeta_{G^{\prime}}\right)$; if $\pi$ has the effect of permuting multiple copies of crossing edges among themselves, it doesn't change the pair $\left(\delta_{G}, \delta_{G^{\prime}}\right)$. The number of distinct pairs $\left(\zeta_{G}, \zeta_{G^{\prime}}\right)$ giving rise to $\Gamma$ is therefore equal to $\Delta ! / Q$, where

$$
Q=\prod_{(u, v) \text { a "crossing edge" }} m(u, v) !=\frac{\Gamma_{e} !}{G_{e} ! G_{e}^{\prime} !}
$$

(the edges we want are those that are in $\Gamma$, but not in either $G$ or $G^{\prime}$ ). Thus our lemma is proved.

Corollary 5.14. The sum over all pairs $\left(\left(G, \delta_{G}\right),\left(G^{\prime}, \delta_{G^{\prime}}\right)\right)$ in $\mathscr{G}_{n, p} \times \mathscr{G}_{m, p}$ such that $<G, G^{\prime}>=\Gamma$, of the inner product $\left\langle I_{G}^{\sim}(\mathbf{f}), I_{G^{\prime}}^{\sim}(\mathbf{g})>\right.$ is equal to

$$
\frac{\Gamma_{v} !}{\Gamma_{e} !} \cdot(-\lambda)^{\left|\Gamma_{\mathscr{T}}\right|} \cdot a(\Gamma) \cdot M_{\Gamma, 2}\left(\mathbf{f}^{*}\right)=I_{\Gamma}\left(\mathbf{f}^{*}\right),
$$

where as elsewhere, $\mathbf{f}^{*}$ is the sequence $g_{m}, g_{m-1}, \ldots, g_{1}, f_{1}, f_{2}, \ldots, f_{n}$.

Proof . Let us begin by taking one pair and working out $\left\langle I^{\sim}{ }_{G}(\mathbf{f}), I^{\sim}{ }_{G^{\prime}}(\mathbf{g})>\right.$. By (5.8) and (5.12) it is equal to $Q_{2} \cdot M_{\Gamma, 2}\left(\mathbf{f}^{*}\right)$ where

$$
Q_{2}=\frac{G_{v}^{+} !\left(G^{\prime}\right)_{v}^{+} !}{G_{e} ! G_{e}^{\prime} !}(-\lambda)^{\left|G_{\mathscr{T}}\right|+\left|G_{\mathscr{T}}^{\prime}\right|} \cdot \frac{1}{\Delta !} a(G) a\left(G^{\prime}\right)
$$

Now $G_{v}^{+}$! is the product of $d_{+}(v)$ ! over $v \in G$. But this is none other than the product of $d(\epsilon(v))$ ! over $\epsilon(v)$ in Bands $m+1 \ldots m+n$ of $\Gamma$, since by $(5.10 .7), d(\epsilon(v))=d_{+}(v)$. Likewise the product $\left(G^{\prime}\right)_{v}^{+}$! is equal to the product of $d(u)$ ! over the remaining vertices $u \in \Gamma$. So, (5.14.2) is

$$
\frac{\Gamma_{v} !}{G_{e} ! G_{e}^{\prime} !}(-\lambda)^{\left|G_{\mathscr{T}}\right|+\left|G_{\mathscr{T}}^{\prime}\right|} \cdot \frac{1}{\Delta !} a(G) a\left(G^{\prime}\right)
$$

But then again, $\left|G_{\mathscr{T}}\right|+\left|G_{\mathscr{T}}^{\prime}\right|$ is equal to $\left|\Gamma_{\mathscr{T}}\right|$ (the number of internal vertices of $\Gamma$ ) and $a(G) \cdot a\left(G^{\prime}\right)=a(\Gamma)$. So,

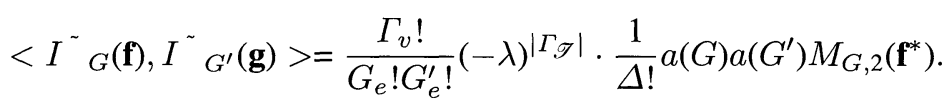

By (5.15.1) we have to sum $\Delta ! G_{e} ! G_{e}^{\prime} ! / \Gamma_{e} !$ such terms, which by (5.14.4) are all equal. The answer is therefore

$$
\frac{\Gamma_{v} !}{\Gamma_{e} !} \cdot(-\lambda)^{\left|\Gamma_{\mathscr{T}}\right|} \cdot a(\Gamma) \cdot M_{\Gamma, 2}\left(\mathbf{f}^{*}\right)=I_{\Gamma}\left(\mathbf{f}^{*}\right)
$$

by (3.5). Thus the lemma is proved. 
Corollary 5.16. The inner product

$$
<\phi_{M}(\mathbf{f}) \Omega, \phi_{M}(\mathbf{g}) \Omega>=W_{n+m}\left(g_{m}, g_{m-1}, \ldots, g_{1}, f_{1}, f_{2}, \ldots, f_{n}\right)
$$

as in (5.1.1); for all sequences $f_{1} \ldots f_{n}, g_{1}, \ldots g_{m}$ in $\mathscr{S}\left(\mathbb{R}^{d+1}\right)$ with $n>0, m>0$.

The proof is now obvious; I think it is also obvious that (5.1.1) holds even when $n$ or $\mathrm{m}$ equals zero (no graph on one side of the great divide; no crossing edges; so one sums over pairs $\left(G, \delta_{G}\right)$ (respectively $\left(G^{\prime}, \delta_{G^{\prime}}\right)$ ), with $\Delta(G)$ (respectively $\Delta\left(G^{\prime}\right)$ ) equal to zero to get $W_{n}$ or $W_{m}$ ). Having established (5.1.1), we bring $\S 5$ to a close.

\section{Hermiticity}

Lemma 6. We have $W_{n}\left(f_{1}, \ldots, f_{n}\right)=\overline{W_{n}\left(f_{n}, f_{n-1}, . ., f_{1}\right)}$, for all sequences $f_{1}, \ldots, f_{n}$ in $\mathscr{S}\left(\mathbb{R}^{d+1}\right)$.

Proof . By (5.1.1), the left hand side is $\left\langle\phi_{M}\left(f_{1}, \ldots, f_{n}\right) \Omega, \Omega>\right.$ and the right hand side is $<\Omega, \phi_{M}\left(f_{1}, \ldots, f_{n}\right) \Omega>$.

\section{Covariance}

The object of this section is to prove the following two results:

Lemma 7.1. If $f_{1}, \ldots, f_{n}$ are in the Schwartz space, and $g$ is an element of the orthochronous Lorentz group $L \uparrow$, then writing $f_{i}^{\prime}=f_{i} \circ g^{-1}(i=1 \ldots n)$ we have

$$
W_{n}\left(f_{1}, \ldots, f_{n}\right)=W_{n}\left(f_{1}^{\prime}, \ldots, f_{n}^{\prime}\right)
$$

Lemma 7.2. If $f_{1}, \ldots, f_{n}$ are in the Schwartz space, and $\tau_{\mathbf{x}}$ denotes a translation $\mathbf{y} \rightarrow \mathbf{y}-\mathbf{x}$ in $\mathbb{R}^{d+1}$ then writing $f_{i}^{\prime}=f_{i} \circ \tau_{\mathbf{x}}$ we again have

$$
W_{n}\left(f_{1}, \ldots, f_{n}\right)=W_{n}\left(f_{1}^{\prime}, \ldots, f_{n}^{\prime}\right)
$$

Together, these will ensure that we have a unitary representation of the Poincare group on the Hilbert space $\mathscr{H}^{0}$, where $\mathscr{H}^{0}$ is the closure of the vectors $\phi_{M}(\mathbf{f}) \Omega$ in $\mathrm{H}$.

Proof of 7.2. Referring to (4.2.1) we find the graphical integrand $M_{G}(\mathbf{f}, \mathbf{p}, \gamma)$ is equal to

$$
\prod_{\imath=1}^{n} \widehat{f}_{i}\left(\phi_{i}(\mathbf{p})\right) \cdot N_{G}(\mathbf{f}, \gamma)
$$

where the only dependence on $\mathbf{f}$ is in the first term. The effect of translating by an amount $\mathbf{x}$ is that for all $\mathbf{q}, \widehat{f}_{i}^{\prime}(\mathbf{q})=\widehat{f}_{i}(\mathbf{q}) \cdot e^{-\mathbf{i q} \cdot \mathbf{x}}$. Hence by (7.2.2),

$$
M_{G}\left(\mathbf{f}^{\prime}, \mathbf{p}, \gamma\right)=\exp \left(-\mathrm{i} \sum_{i=1}^{n} \phi_{\imath}(\mathbf{p}) \cdot \mathbf{x}\right) M_{G}(\mathbf{f}, \mathbf{p}, \gamma)=M_{G}(\mathbf{f}, \mathbf{p}, \gamma)
$$

because, of course, the sum of all the flows $\phi_{i}(\mathbf{p})$ contains one entry $\mathbf{p}_{e}$ and one entry $-\mathbf{p}_{e}$ for each edge $e \in e_{X}(G)$, and is zero. Hence, the graphical integrals $I_{G}(\mathbf{f})$ and $I_{G}\left(\mathbf{f}^{\prime}\right)$ are all equal, so $W_{n}(\mathbf{f})=W_{n}\left(\mathbf{f}^{\prime}\right)$. 
Proof of 7.1. The effect of the substitution $f_{i}^{\prime}=f_{i} \circ g^{-1}$ is that

$$
\widehat{f}_{i}^{\prime}(\mathbf{q})=\widehat{f}_{i}\left(g^{*} \mathbf{q}\right)
$$

where $g^{*}$ denotes the transpose of the linear map $g$. Let us now consider the graphical integrand $M_{G}\left(\mathbf{f}^{\prime}, \mathbf{p}^{\prime}, \boldsymbol{\gamma}^{\prime}\right)$ where we define

$$
\mathbf{p}^{\prime}=\left(\mathbf{p}_{e}^{\prime}\right)_{e \in e(G)} ; \quad \mathbf{p}_{e}^{\prime}= \begin{cases}\mathbf{p}_{e} & \text { if } e \text { lies within a single band of } G ; \\ \left(g^{*}\right)^{-1} \mathbf{p}_{e} & \text { if } e \in e_{X}(G)\end{cases}
$$

and

$$
\gamma^{\prime}=\left(\gamma_{e}^{\prime}\right)_{e \in e_{X}(G)}=\left(\gamma_{e} g^{*}\right)_{e \in e_{X}(G)} .
$$

We claim that when $\mathbf{f}, \mathbf{p}$ and $\gamma$ are as above,

$$
M_{G}\left(\mathbf{f}^{\prime}, \mathbf{p}^{\prime}, \gamma^{\prime}\right)=M_{G}(\mathbf{f}, \mathbf{p}, \gamma)
$$

For looking at Definitions (3.1.2-7) we note first that the Lorentz transform $\left(g^{*}\right)^{-1}$ does not change the value of $\mathbf{p}_{e}^{2}$, hence

$$
\left(\mathbf{p}_{e}^{\prime}\right)^{2}=\mathbf{p}_{e}^{2}
$$

for all $e \in e(G)$, and all factors $\mu\left(\mathbf{p}_{e}^{2}\right)$ are unaffected by the map $\mathbf{p} \rightarrow \mathbf{p}^{\prime}$. Second, all factors $\widehat{f}_{\imath}^{\prime}(\phi)$ have the fourier transform evaluated at $\phi=\phi_{i}\left(\mathbf{p}^{\prime}\right)$. Every edge $\mathbf{p}_{e}$ involved in the flow into band $i$ must, obviously, cross between two bands; hence, (7.1.4) gives $\phi_{i}\left(\mathbf{p}^{\prime}\right)=\left(g^{*}\right)^{-1} \phi_{i}(\mathbf{p})$ so

$$
\widehat{f}_{i}^{\prime}\left(\phi_{i}^{\prime}\left(\mathbf{p}^{\prime}\right)\right)=\widehat{f}_{i}^{\prime}\left(\left(g^{*}\right)^{-1} \phi_{i}(\mathbf{p})\right)=\widehat{f}_{i}(\mathbf{p})
$$

by (7.1.2). Thus, factors $\widehat{f}_{\imath}\left(\phi_{i}(\mathbf{p})\right)$ are unaffected by the substitutions $\mathbf{f} \rightarrow \mathbf{f}^{\prime}, \mathbf{p} \rightarrow \mathbf{p}^{\prime}$.

Thirdly, all factors $\widehat{\theta}(\mathbf{q})$ in (3.1.2-7) are evaluated either at $\mathbf{q}=g_{e} \mathbf{p}_{e}$ for an edge $e \in e_{X}(G)$, or at $\mathbf{p}_{e}$ for an edge $e$ lying within a band. In either case, the value of $\mathbf{q}$ is unaffected by the changes we have made, the nontrivial case being when $e \in e_{X}(G)$ so $\mathbf{p}_{e}{ }^{\prime}=\left(g^{*}\right)^{-1} \mathbf{p}_{e}$, but on the other hand, $\gamma_{e}^{\prime}=\gamma_{e} g^{*}$ so

$$
\gamma_{e}^{\prime} \mathbf{p}_{e}{ }^{\prime}=\gamma_{e} \mathbf{p}_{e} .
$$

Lastly, most of (3.1.2-7) have dependence on the sign of $L_{e}$ via the step-functions $\mathrm{H}\left(L_{e}\right)$; but the factors $\mu\left(\mathbf{p}_{e}^{2}\right)$ ensure that the contribution is zero unless $\mathbf{p}_{e}$ is timelike; in which case the sign of $L_{e}$ cannot be changed by any orthochronous Lorentz transform $g$. This concludes the proof of (7.1.4), and the rest is easy:

We claim the first integrated moment

$$
M_{G, 1}\left(\mathbf{f}^{\prime}, \gamma^{\prime}\right)=M_{G, 1}(\mathbf{f}, \gamma)
$$

The only thing needing remark is that the delta functions of (3.3.1) are unchanged by the substitutions we have made. For the linear functionals $\psi_{i, j}(\mathbf{p})$ are linear combinations of momenta $\mathbf{p}_{e}$ such that the edge $e$ is internal to a particular band, and momenta $\gamma_{e} \mathbf{p}_{e}$ such that $e \in e_{X}(G)$ (see (3.2)). As we have already remarked, both these quantities remain unchanged when $\mathbf{p} \rightarrow \mathbf{p}^{\prime}$ and $\gamma \rightarrow \gamma^{\prime}$.

Next, we claim that the second integrated moment

$$
M_{G, 2}(\mathbf{f})=M_{G, 2}\left(\mathbf{f}^{\prime}\right) .
$$


Proof. By (3.4) this is just a matter of integrating (7.1.8) with respect to $\gamma$ on the left and $\gamma^{\prime}$ on the right, noting that the two forms of integration are the same because $d \gamma_{e}$ is always right-invariant Haar measure, invariant under any transformation $\gamma_{e} \rightarrow \gamma_{e} g^{*}$.

But now we need only take a finite linear combination of our results as in (3.5) to conclude that $W_{n}(\mathbf{f})=W_{n}\left(\mathbf{f}^{\prime}\right)$; on which happy note we conclude $\S 7$.

\section{Spectral Properties and Vacuum Uniqueness}

In $\S 5$ we defined vectors $\phi_{M}\left(f_{1}, \ldots, f_{n}\right)$ in a Hilbert space $\mathscr{H}$, whose inner products are the Wightman functions of our theory. The vectors $\phi_{M}\left(f_{1}, \ldots, f_{n}\right)$ are certainly not dense in $\mathscr{H}$, so the time has come to restrict to a subspace of $\mathscr{H}$ in which they are.

Definition 8.1. Let $\mathscr{H} \mathscr{C}^{0} \subset \mathscr{H}$ be the closed linear span of the $\operatorname{set}\left\{\phi_{M}\left(f_{1}, \ldots, f_{n}\right) \Omega\right.$ : $\left.n \geq 0, f_{1}, \ldots, f_{n} \in \mathscr{S}\left(\mathbb{R}^{d+1}\right)\right\}$.

Let us summarise our results (Hermiticity, Covariance) so far:

\section{Theorem 8.2.}

(a) For each $f \in \mathscr{S}\left(\mathbb{R}^{d+1}\right)$, the map $f(\phi)$ which sends $\phi_{M}\left(f_{1}, \ldots, f_{n}\right) \Omega$ to $\phi_{M}\left(f, f_{1}, \ldots, f_{n}\right) \Omega$ extends linearly to a densely defined Hermitian operator on $\mathscr{H}^{0}$ whose domain of definition includes the vectors $\phi_{M}(\mathbf{f}) \Omega$.

(b) For each element $g=h \circ \tau$ in the Poincaré group $P(h \in L \uparrow, \tau$ a translation on $\left.\mathbb{R}^{d+1}\right)$ the map $U_{g}$ which sends $\phi_{M}\left(f_{1}, \ldots, f_{n}\right) \Omega$ to $\phi_{M}\left(f_{1} \circ g^{-1}, f_{2} \circ g^{-1}, \ldots, f_{n} \circ g^{-1}\right) \Omega$ (8.2.1) extends linearly to a unitary operator on $\mathscr{H} \mathscr{C}^{0}$; and the collection $\left\{U_{g}, g \in P\right\}$ is a unitary representation of the Poincaré group.

Note. the presence of the inverses in (8.2.1) means we are picturing the Poincare group as having its maps written on the right; the author prefers to picture a delta-function $f_{1}$ moving up the positive time axis under positive time translation.

The proof of these facts comes straightforwardly from the results of $\S \S 6-7$; for the details, see the proof of the Wightman reconstruction theorem [SW1, p.117]. One of the known (see $\S 4$ ) properties of the $W_{n}$ is that they are continuous functions of $f_{1}, \ldots, f_{n}$ on Schwartz space; hence the representation in (b) is strongly continuous because it is a fact that, as $g_{\imath} \rightarrow g$ in $P$ so $f \circ g_{i} \rightarrow f \circ g$ in $\mathscr{S}\left(\mathbb{R}^{d+1}\right)$; from which we may infer convergence of various Wightman functions, and deduce strong continuity rather easily. So looking at the subgroup of translations on $\mathbb{R}^{d+1}$, we have a strongly continuous unitary representation of $\mathbb{R}^{d+1}$ under addition; which must therefore have an infinitesimal generator $\left(\mathbf{P}, P_{0}\right)$ consisting of $d+1$ densely defined and mutually commuting Hermitian operators on $\mathscr{H} \mathscr{C}^{0}$ (here $P_{0}$ is of course the time coordinate, the others being $P_{1} \ldots P_{d}$ ). We are now going to investigate the joint spectrum of the $P_{i}$, and prove the following theorem:

Theorem 8.3. The joint spectrum of $\left(\mathbf{P}, P_{0}\right)$ lies entirely in the forward cone $P_{0}^{2}-\mathbf{P}^{2} \geq$ $0, P_{0} \geq 0$.

A closely related theorem which will also be proved in this section is the following:

Theorem 8.4. If $\Omega^{\perp}$ denotes the vector subspace of $\mathscr{H}^{0}$ consisting of vectors orthogonal to the vacuum, then $\Omega^{\perp}$ is an invariant subspace of $\mathscr{H} \mathscr{C}^{0}$, and the spectrum of the restricted operator $\left.P_{0}\right|_{\Omega^{\perp}}$ is confined to the region $P_{0} \geq m_{0}$, where $m_{0}$ is, as previously, the lower end of the support of our probabilistic mass density $\mu$. 
This theorem, once proved, ensures not only that our theories have unique vacuum, but also the existence of a "mass gap" $m_{0}$ between zero and the first nontrivial spectrum of the Hamiltonian $P_{0}$.

Proof of (8.3) and (8.4). The method of proof is to identify each component $P_{k}$ with the restriction to the subspace $\mathscr{H} \mathscr{C}^{0}$ of a very well known operator $Q_{k}$ on $\mathscr{H}$. Recall that $\mathrm{H}$ is the $L_{2}$ direct sum of Hilbert spaces $\mathscr{H}_{\Delta}$ where $\mathscr{H}_{\Delta}$ is $L_{2}\left(\left(\mathbb{R}^{d+1} \times L\right)^{\Delta}\right)$. On the space $L_{2}\left(\left(\mathbb{R}^{d+1} \times L\right)^{\Delta}\right)$ there is a densely defined Hermitian operator $Q_{k}^{\Delta}$ such that for functions $F(\mathbf{q}, \boldsymbol{\eta})=F\left(\mathbf{q}_{1}, \eta_{1} ; \mathbf{q}_{2}, \eta_{2} ; \ldots, \mathbf{q}_{\Delta}, \eta_{\Delta}\right)\left(\mathbf{q}_{i} \in \mathbb{R}^{d+1}, \eta_{i} \in L\right)$ we have

$$
\left(Q_{k}^{\Delta} F\right)[\mathbf{q}, \boldsymbol{\eta}]=\left(\sum_{i=1}^{\Delta} \mathbf{q}_{i}\right) \cdot \mathbf{e}_{k} F[\mathbf{q}, \boldsymbol{\eta}] .=\left(\sum_{i=1}^{\Delta} q_{i}^{k}\right) F[\mathbf{q}, \boldsymbol{\eta}]
$$

(where $\mathbf{e}_{k}$ is the $k$ th unit vector). We will now write $Q_{k}$ to denote the densely defined operator on $\mathrm{H}$ which coincides with (8.3.2) on each $\mathscr{H}_{\Delta}(\Delta>0)$ and is zero on $\mathscr{H}_{0}=\mathbb{C}$. We claim that $P_{k}$ is none other than the restriction to $\mathscr{H}^{0}$ of $Q_{k}(i=1, \ldots . \Delta)$. It is enough to show that for all $\rho \in \mathbb{R}, e^{\mathrm{i} \rho P_{k}}$ is equal to $\left.e^{\mathrm{i} \rho Q_{k}}\right|_{\mathscr{H} O}$. Now $e^{\mathrm{i} \rho P_{k}}$ is $U_{\tau}$ where $\tau$ is a translation by an amount $\rho$ in the $\mathbf{e}_{k}$ direction.

Pick any sequence $\mathbf{f}=f_{1}, \ldots, f_{n}$ in $\mathscr{S}\left(\mathbb{R}^{d+1}\right)$, any element $\left(G, \delta_{G}\right)$ of $\mathscr{G}_{n, p}$, and any sequences $\mathbf{p}=\left(\mathbf{p}_{e}, e \in e(G)\right)$ in $\mathbb{R}^{d+1}$ and $\gamma=\left(\gamma_{e}, e \in e_{X}(G)\right)$ in L. Write $\Delta=\Delta(G)$ and by $(5.3 .6)$,

$$
M_{G}^{\sim}(\mathbf{f}, \mathbf{p}, \gamma)[\mathbf{q}, \boldsymbol{\eta}]=\prod_{i=1}^{n} \widehat{f}_{i}\left(\tilde{\phi}_{\imath}(\mathbf{p}, \mathbf{q})\right) \cdot N_{G}{ }_{G}(\mathbf{p}, \gamma)
$$

where $N^{\sim}{ }_{G}$ does not depend on $\mathbf{f}$. When each $f_{\imath}$ is replaced by $f_{i} \circ \tau$ (giving a sequence $\mathbf{f} \circ \tau$ say), we therefore have

$$
M_{G}(\mathbf{f} \circ \tau, \mathbf{p}, \gamma)[\mathbf{q}, \boldsymbol{\eta}]=M_{G}^{\sim}(\mathbf{f}, \mathbf{p}, \gamma)[\mathbf{q}, \boldsymbol{\eta}] \cdot \exp \left(-\mathrm{i} \rho \sum_{i=1}^{n} \tilde{\phi}_{i}(\mathbf{p}, \mathbf{q}) \cdot \mathbf{e}_{k}\right) \text {. }
$$

Now each $\phi^{\sim}{ }_{i}$ is, by (5.3.2), equal to $\phi_{i}(\mathbf{p})-\sum_{\delta_{G}(j) \in B_{i}(G)} \mathbf{q}_{j}$ (8.3.5) where $\phi_{i}$ is the flow into band $i$ in the graph $G$. In (8.3.4), however, we are only interested in the sum over all $\mathrm{i}$; and certainly $\sum_{i=1}^{n} \phi_{i}(\mathbf{p})=0$ (8.3.6). Also, the sum

$$
\sum_{\imath=1}^{n} \sum_{\delta_{G}(j) \in B_{\imath}(G)} \mathbf{q}_{j}=\sum_{i=1}^{\Delta} \mathbf{q}_{i}
$$

because each $\delta_{G}(j)$ is in one and only one band of $G$. Therefore (8.3.4) gives

$$
M_{G}^{\sim}(\mathbf{f} \circ \tau, \mathbf{p}, \gamma)[\mathbf{q}, \boldsymbol{\eta}]=e^{\left(\mathrm{i} \rho \sum_{i=1}^{\Delta} q_{i}^{k}\right)} M_{G}^{\sim}(\mathbf{f}, \mathbf{p}, \gamma)[\mathbf{q}, \boldsymbol{\eta}] .
$$

Integrating up as in (5.5-7) we get

$$
I_{G}(\mathbf{f} \circ \tau)[\mathbf{q}, \boldsymbol{\eta}]=e^{\left(\mathrm{i} \rho \sum_{i=1}^{\Delta} q_{\imath}^{k}\right)} I_{G}^{\sim}(\mathbf{f})[\mathbf{q}, \boldsymbol{\eta}],
$$

hence by (5.8), we may write $\phi_{M}(\mathbf{f}) \Omega=c+\sum_{i=1}^{n S} h_{\imath} \quad\left(c \in \mathbb{C}=\mathscr{T}_{0} ; h_{i} \in \mathscr{H}_{i}\right)$ and $\phi_{M}(\mathbf{f} \circ \tau) \Omega=c^{\prime}+\sum_{i=1}^{n S} h_{i}^{\prime}\left(c^{\prime} \in \mathbb{C}=\mathscr{H}_{0} ; h_{i}^{\prime} \in \mathscr{H}_{i}\right)$. We find that $c^{\prime}=c$ and for each $\Delta>0$, 


$$
h_{\Delta}^{\prime}[\mathbf{q}, \boldsymbol{\eta}]=e^{\left(\mathrm{i} \rho \sum_{i=1}^{\Delta} q_{\imath}^{k}\right)} h_{\Delta}[\mathbf{q}, \boldsymbol{\eta}]
$$

Hence,

$$
\phi_{M}(\mathbf{f} \circ \tau) \Omega=e^{\mathrm{i} \rho Q_{k}} \phi_{M}(\mathbf{f}) \Omega
$$

that is,

$$
e^{\mathrm{i} \rho P_{k}} \phi_{M}(\mathbf{f}) \Omega=e^{\mathrm{i} \rho Q_{k}} \phi_{M}(\mathbf{f}) \Omega,
$$

and our Claim is established. But it is now almost trivial to get $(8.3,4)$. For the $Q_{k}$ are only pointwise multiplication operators on the $L_{2}$ spaces involved, so their spectral subspaces are rather easy to identify; they consist of all functions supported on the region where you are "pointwise multiplying" by a number in the given set.

Hence it is enough to establish (8.3), if for a set of elements $\zeta \in \mathscr{H} \mathscr{C}^{0}$ whose linear span is dense, we can write

$$
\boldsymbol{\zeta}=c+\sum_{i=1}^{\infty} h_{\imath}\left(c \in \mathbb{C}, h_{i} \in \mathscr{H}_{i}\right)
$$

with every $h_{\Delta}$ supported on the set $\left\{(\mathbf{q}, \boldsymbol{\eta}): \mathbf{q}_{i}^{2} \geq 0, q_{i}^{0} \geq 0 ; i=1, \ldots . \Delta\right\}$. No prizes for guessing our choice of set with dense linear span; of course, it's the $\phi_{M}(\mathbf{f}) \Omega$. Inspecting (5.3.4), (5.3.5) we actually do rather better than (8.3.12); for each $i=1, \ldots . \Delta$ there are invariably factors $\mathrm{H}\left(q_{i}^{0}\right) \mu\left(\mathbf{q}_{i}^{2}\right)$ in each $M_{G}^{\sim(i)}$; hence if $\zeta$ is a vector $\phi_{M}(\mathbf{f}) \Omega$, it can be written as in (8.3.12) but with all $h_{\Delta}(\Delta>0)$ supported on

$$
\left\{[\mathbf{q}, \boldsymbol{\eta}]: \mathbf{q}_{i}^{2} \geq m_{0}^{2}, q_{i}^{0} \geq m_{0}, i=1, \ldots \Delta\right\} .
$$

This implies not only that $P_{0} \geq 0$ and $P_{0}^{2}-\mathbf{P}^{2} \geq 0$ but also that the operator

$$
\left.P_{0}\right|_{\mathscr{H}_{1} \oplus \cdot \mathscr{H}_{2} \oplus \mathscr{H}_{3} \oplus \ldots}=\left.P_{0}\right|_{\Omega^{\perp}} \geq m_{0} \cdot I,
$$

thus establishing (8.4) as well. Thus, $\S 8$ is brought to a close.

\section{Locality}

The aim of this section is to prove the following result:

Lemma 9. If $f_{1}, \ldots, f_{n}$ are in the Schwartz space $\mathscr{S}\left(\mathbb{R}^{d+1}\right)$, and if the supports of the functions $f_{j}$ and $f_{j+1}$ are spacelike separated, then

$$
W_{n}\left(f_{1}, \ldots, f_{n}\right)=W_{n}\left(f_{1}, \ldots, f_{\jmath-1}, f_{j+1}, f_{j}, f_{j+2}, f_{j+3}, \ldots, f_{n}\right) .
$$

From this lemma we shall immediately obtain the corollary:

Corollary 9.1. If $f$ and $g$ are in the Schwartz space, and if the supports of $f$ and $g$ are spacelike separated, then the operators $\phi_{M}(f)$ and $\phi_{M}(g)$ (as in (8.2)) commute.

For the proof of this given Lemma 9, see [SW1].

Since the local commutativity axiom is in fact the last of the Wightman axioms that need verifying for our functions $W_{n}$, we then will immediately deduce:

Theorem 9.2. The functions $W_{n}$ defined in $\S 3$, are indeed the Wightman functions of a hermitian scalar field theory. ered.

In Section 10 we will begin to discuss the nature of the theories we have discov- 
Proof of Lemma 9. We begin by "nibbling" at the problem as follows:

Lemma 9.3. In order to establish (9.0), it is sufficient to prove that (9.0) holds whenever the functions $f_{j}$ and $f_{j+1}$ have spacelike separated compact supports.

Proof. The functions $W_{n}(\mathbf{f})$ are continuous in their arguments (Lemma 4.3), and it is a fact that the pairs $\left(f_{j}, f_{j+1}\right)$ in Schwartz space such that their supports are compact and spacelike separated are dense in the set of all pairs $\left(f_{\jmath}, f_{j+1}\right)$ with spacelike separated (but not necessarily compact) supports. Thus (9.3) is established.

Another small bite is taken out of the problem as follows:

Lemma 9.4. In order to establish (9.0), it is sufficient to show that (9.0) holds in the case when $f_{j}$ and $f_{j+1}$ have compact supports which are "spacelike separated in the $\mathbf{e}_{1}$ direction," that is, there is a $v<1$ such that for all $\mathbf{x} \in \operatorname{supp} f_{j}$ and $\mathbf{x}^{\prime} \in \operatorname{supp} f_{j+1}$ we have

$$
\left|x_{0}-x_{0}^{\prime}\right|<v\left|x_{1}-x_{1}^{\prime}\right|
$$

Proof . [One might be tempted to say "by an elementary compactness argument"; but at any rate, here is the compactness argument:] Write $f=f_{j}$ and $f^{\prime}=f_{j+1}$. We may, by (9.3), assume that $f$ and $f^{\prime}$ have compact supports. For every $\mathbf{x} \in \operatorname{supp} f$ and $\mathbf{x}^{\prime} \in \operatorname{supp} f^{\prime}$, the vector $\mathbf{x}-\mathbf{x}^{\prime}$ is spacelike, (that is, there is a unit vector $\mathbf{u}$ with zero time coordinate, and a speed $v<1$, such that

$$
\left|x_{0}-x_{0}^{\prime}\right|<v\left|\left(\mathbf{x}-\mathbf{x}^{\prime}\right) \cdot \mathbf{u}\right| \text {. }
$$

Now the set of points $\left(\mathbf{x}, \mathbf{x}^{\prime}\right)$ such that (9.4.2) holds for given $\mathbf{u}$ and $v$, is open in $\mathbb{R}^{2(d+1)}$. So, for some $\eta>0$, (9.4.2) also holds if $\left(\mathbf{x}, \mathbf{x}^{\prime}\right)$ is replaced by $\left(\mathbf{y}, \mathbf{y}^{\prime}\right)$ with $\|\mathbf{y}-\mathbf{x}\|<\eta,\left\|\mathbf{y}^{\prime}-\mathbf{x}^{\prime}\right\|<\eta$, [where $\|\cdot\|$ denotes the positive definite quantity as before]. Let us write $B\left(\mathbf{x}, \mathbf{x}^{\prime}, \eta\right)$ for the set

$$
\left\{\left(\mathbf{y}, \mathbf{y}^{\prime}\right) \in \mathbb{R}^{2(d+1)}:\|\mathbf{y}-\mathbf{x}\|<\eta,\left\|\mathbf{y}^{\prime}-\mathbf{x}^{\prime}\right\|<\eta\right\} .
$$

The compact set supp $f \times \operatorname{supp} f^{\prime}$ is therefore covered by open sets $B\left(\mathbf{x}, \mathbf{x}^{\prime}, \eta / 2\right)$ $\left(\eta=\eta\left(\mathbf{x}, \mathbf{x}^{\prime}\right)\right.$ depending on $\mathbf{x}$ and $\left.\mathbf{x}^{\prime}\right)$ such that the following holds. For all $\left(\mathbf{x}, \mathbf{x}^{\prime}\right) \in$ supp $f \times \operatorname{supp} f^{\prime}$ there is a unit vector $\mathbf{u}=\mathbf{u}\left(\mathbf{x}, \mathbf{x}^{\prime}\right)$ and speed $v=v\left(\mathbf{x}, \mathbf{x}^{\prime}\right)<1$ such that

$$
\left|y_{0}-y_{0}^{\prime}\right|<v\left|\left(\mathbf{y}-\mathbf{y}^{\prime}\right) \cdot \mathbf{u}\right|
$$

for all $\left(\mathbf{y}, \mathbf{y}^{\prime}\right) \in B\left(\mathbf{x}, \mathbf{x}^{\prime}, \eta\left(\mathbf{x}, \mathbf{x}^{\prime}\right)\right)$. There is therefore a finite subcover, say involving balls $B\left(\mathbf{x}_{i}, \mathbf{x}_{i}^{\prime}, \eta_{i} / 2\right)(i=1, \ldots M)$, unit vectors $\mathbf{u}_{i}$ and speeds $v<1$. Define $\eta=$ $\min \left\{\eta_{i}, i=1 . . M\right\}$ and $v=\max \left\{v_{\imath}, i=1 . . M\right\}$. Then for any $\left(\mathbf{x}, \mathbf{x}^{\prime}\right) \in \operatorname{supp} f \times$ supp $f^{\prime}$ there is a unit vector $\mathbf{u}$ such that (9.4.4) holds, for any $\left(\mathbf{y}, \mathbf{y}^{\prime}\right) \in B\left(\mathbf{x}, \mathbf{x}^{\prime}, \eta / 2\right)$; for we just choose $\mathbf{u}=\mathbf{u}_{i}$, where $\left(\mathbf{x}, \mathbf{x}^{\prime}\right) \in B\left(\mathbf{x}_{i}, \mathbf{x}_{i}^{\prime}, \eta / 2\right)$.

Now we can write $f=\sum_{i=1}^{R} g_{i}, f^{\prime}=\sum_{i=1}^{R^{\prime}} g_{i}^{\prime}$, where each $g_{\imath}\left(g_{i}^{\prime}\right)$ is supported on a subset of supp $f\left(\operatorname{supp} f^{\prime}\right)$ of diameter at most $\eta / 2$. Then, since

$$
W_{n}\left(f_{1}, \ldots, f_{n}\right)=\sum_{i=1}^{R} \sum_{k=1}^{R^{\prime}} W_{n}\left(f_{1}, . ., f_{j-1}, g_{i}, g_{k}^{\prime}, f_{j+2}, . ., f_{n}\right)
$$

and the analogous statement is also true with $f_{\jmath}$ and $f_{\jmath+1}$ swapped, $g_{i}$ and $g_{k}^{\prime}$ swapped, and $G^{\prime}$ replacing $G$; we find it is enough to prove (9.0) in the case when $f_{j}$ and $f_{j+1}$ 
are supported on sets such that for some fixed unit vector $\mathbf{u}$ and speed $v<1$, we have

$$
\left|x_{0}-x_{0}^{\prime}\right|<v\left|\left(\mathbf{x}-\mathbf{x}^{\prime}\right) \cdot \mathbf{u}\right|
$$

for all $\left(\mathbf{x}, \mathbf{x}^{\prime}\right) \in \operatorname{supp} f_{j} \times \operatorname{supp} f_{j+1}$. Our functions $W_{n}$ are known to be covariant, so they are certainly invariant under space rotations. Therefore we may assume $\mathbf{u}=\mathbf{e}_{1}$; and this concludes the proof.

We now proceed to the heart of the matter.

We note that if an equation like (9.0) holds at all, it is more or less bound to hold "graph by graph"; so we now make a specific assertion concerning which graph gets "paired off" with which.

Definition 9.5. With the notation of (9.0) let $\mathbf{f}$ denote the sequence $f_{1}, \ldots, f_{n}$; and let $\mathbf{f}^{\prime}$ denote the sequence $f_{1}, \ldots, f_{j-1}, f_{j+1}, f_{j}, f_{j+2}, f_{j+3}, \ldots, f_{n}$. Thus,

$$
f_{\imath}^{\prime}=f_{i \circ \tau},
$$

where $\tau$ is the transposition permutation $(j, j+1)$ acting on the set $\{1,2, . ., n\}$.

The transposition $\tau$ is going to play a role similar to that of the map $\epsilon$ in $\S 5$, only less complicated.

Definition 9.6. Let $G \in \mathscr{G}_{n}$ be any graph. We define a graph $G^{\prime}$ as follows:

$G^{\prime}$ has vertex set $\left\{F_{i}, i=1, \ldots . n\right\} \cup\left\{X_{i, j}: i=1, \ldots . n, j=1, \ldots . s_{i}^{\prime}\right\}$; where

$$
s_{i}^{\prime}=s_{\tau(i)} \text {; }
$$

and $s_{i}=s_{i}(G)$ is the analogous sequence for the graph $G$. We define the natural map $\tau: G \rightarrow G^{\prime}$ by

$$
\tau\left(F_{i}\right)=F_{\tau(i)}, \quad \tau\left(X_{i, j}\right)=X_{\tau(i), j} .
$$

The edges of $G^{\prime}$ are the edges $\tau(e)=(\tau(u), \tau(v))$ such that $e=(u, v)$ is an edge of $G$ (counted of course according to multiplicity).

The point of Definition 9.6 is of course the following:

Lemma 9.7. The map $\tau$ defines a bijection $\mathscr{G}_{n} \rightarrow \mathscr{G}_{n}$ and if the supports of $f_{j}$ and $f_{j+1}$ are compact and spacelike separated in the $\mathbf{e}_{1}$ direction, then for each $G \in \mathscr{O}_{n}$ we have

$$
M_{G^{\prime}, 2}\left(\mathbf{f}^{\prime}\right)=M_{G, 2}(\mathbf{f}) ;
$$

where $\mathbf{f}, \mathbf{f}^{\prime}$ are as in (9.5), and $G^{\prime}=\tau(G)$.

In view of (3.4) and (3.5), Lemma 9 is proved once (9.7) is established.

Definition 9.8. Let $f_{1}, \ldots, f_{n}, v$ be as in (9.4), with (9.4.1) holding for each $\mathbf{x} \in$ supp $f_{j}$, and each $\mathbf{x}^{\prime} \in \operatorname{supp} f_{\jmath+1}$. The function $\gamma: \mathbb{R} \times \mathbb{R}^{2} \times \mathbb{R}^{d-1} \times \mathbb{R} \rightarrow \mathbb{R}$ is defined by

$$
\gamma(\alpha, \mathbf{x}, \boldsymbol{\xi}, \rho)=|\rho| f_{\jmath+1}\left(x_{0}-\rho \sinh \alpha, x_{1}+\rho \cosh \alpha, \xi_{2}, \xi_{3}, \ldots, \xi_{d}\right),
$$

where $\mathbf{x}=\left(x_{0}, x_{1}\right) ; \boldsymbol{\xi}=\left(\xi_{2}, \xi_{3} . ., \xi_{d}\right)$. By convention fourier transforms of this function will be done on the $\rho$-coordinate only; thus, we define

$$
\widehat{\gamma}(\alpha, \mathbf{x}, \boldsymbol{\xi}, \omega)=\int_{-\infty}^{\infty} \gamma(\alpha, \mathbf{x}, \boldsymbol{\xi}, \rho) e^{-1 \omega \rho} d \rho
$$


Lemma 9.9. If $\left(x_{0}, x_{1}\right)$ is in the set of pairs such that there is a vector of form $\left(x_{0}, x_{1}, \ldots, x_{d}\right) \in \operatorname{supp} f_{j}$, then the function

$$
\rho \rightarrow \gamma\left(\alpha,\left(x_{0}, x_{1}\right), \boldsymbol{\xi}, \rho\right)
$$

is in the Schwartz space $\mathscr{S}(\mathbb{R})$, is uniformly bounded as $x_{0}, x_{1}$ and $\alpha$ vary, and is zero for large enough $\left|x_{0}\right|+\left|x_{1}\right|+|\alpha|+\|\boldsymbol{\xi}\|$.

Proof . Concerning membership of the Schwartz space, the only doubtful thing is whether we have infinite differentiability near $\rho=0$. But if $\rho=0,(9.4 .1)$ ensures that the vector $\left(x_{0}-\rho \sinh \alpha, x_{1}+\rho \cosh \alpha, \xi_{2}, . ., \xi_{d}\right)$ is not in supp $f_{j+1}$, so $\gamma$ and all its derivatives are zero on a neighbourhood of the point.

Concerning uniform boundedness, we note that actually $x_{0}, x_{1}$ only vary within a compact set, and furthermore, $\gamma$ is zero for $\tanh \alpha>v$ by (9.4.1). Since $f_{j+1}$ is a compactly supported element of the Schwartz space, the result follows.

We now rewrite the product $\widehat{f}_{j}(\mathbf{q}) \widehat{f}_{j+1}\left(\mathbf{q}^{\prime}\right)\left(\mathbf{q}, \mathbf{q}^{\prime} \in \mathscr{S}\left(\mathbb{R}^{d+1}\right)\right)$ in the following way:

$$
\begin{aligned}
\widehat{f}_{j}(\mathbf{q}) \cdot \widehat{f}_{\jmath+1}\left(\mathbf{q}^{\prime}\right) & =\int_{\text {supp } f_{j} \times \mathbb{R}^{d+1}} f_{j}(\mathbf{x}) f_{j+1}(\boldsymbol{\xi}) \exp \left(-\mathrm{i}\left(\mathbf{q} \cdot \mathbf{x}+\mathbf{q}^{\prime} \cdot \boldsymbol{\xi}\right)\right) d \mathbf{x} d \boldsymbol{\xi} \\
& =\int_{E} f_{j}(\mathbf{x}) f_{\jmath+1}\left(x_{0}-\rho \sinh \alpha, x_{1}+\rho \cosh \alpha, \xi_{2} . . \xi_{d}\right) \times \\
\exp (-\mathrm{i}(\mathbf{q} \cdot \mathbf{x} & \left.+\mathbf{q}^{\prime} \cdot\left(x_{0}-\rho \sinh \alpha, x_{1}+\rho \cosh \alpha, \xi_{2} . . \xi_{d}\right)\right)|\rho| d \alpha d \rho d \boldsymbol{\xi} d \mathbf{x}
\end{aligned}
$$

where $E=\left[-\tanh ^{-1} v, \tanh ^{-1} v\right] \times \mathbb{R} \times \mathbb{R}^{d-1} \times \operatorname{supp} f_{j}$

$$
\begin{gathered}
=\int_{E^{\prime}} f_{j}(\mathbf{x}) \widehat{\gamma}\left(\alpha,\left(x_{0}, x_{1}\right), \boldsymbol{\xi}, q_{1}^{\prime} \cosh \alpha-q_{0}^{\prime} \sinh \alpha\right) \times \\
\exp \left(-\mathrm{i}\left(\mathbf{q} \cdot \mathbf{x}+\mathbf{q}^{\prime} \cdot\left(x_{0}-\rho \sinh \alpha, x_{1}+\rho \cosh \alpha, \xi_{2} . . \xi_{d}\right)\right) d \alpha d \boldsymbol{\xi} d \mathbf{x},\right.
\end{gathered}
$$

where $E^{\prime}=\left[-\tanh ^{-1} v, \tanh ^{-1} v\right] \times \mathbb{R}^{d-1} \times \operatorname{supp} f_{j}$.

Note. We would like to substitute our slightly peculiar formula (9.10.2) into the moments $M_{G}(\mathbf{f}, \mathbf{p}, \mathbf{g})$ and $M_{G^{\prime}}\left(\mathbf{f}^{\prime} \mathbf{p}^{\prime} \mathbf{g}^{\prime}\right)$ (which, as the reader will recall, involve factors $\widehat{f}_{j}\left(\phi_{j}(\mathbf{p})\right)$ and $\widehat{f}_{j+1}\left(\phi_{j+1}^{\prime}\left(\mathbf{p}^{\prime}\right)\right)$, where $\phi_{j}$ and $\phi_{j+1}^{\prime}$ denote the flows within $G$ and $G^{\prime}$ respectively; and we would then like to integrate up to get the second moments $M_{G, 2}$, reversing the orders of integration freely along the way. This however is illegal, because the multiple integral would not be absolutely convergent. So we take a slightly different route:

Definition 9.11. For each speed $u \in[0,1)$ we define a subset $L_{u} \subset L$ as follows:

$L_{u}=\left\{ \pm U \cdot \Lambda_{\mathbf{v}}: U\right.$ is a space rotation, and $\Lambda_{\mathbf{v}}$ a velocity boost of speed at most $\left.u\right\}$.

We then define the subgroup $L_{01} \subset L$ consisting of all Lorentz transforms (including time reversal) which act on $\operatorname{lin}\left\{\mathbf{e}_{0}, \mathbf{e}_{1}\right\}$ and leave $\operatorname{lin}\left\{\mathbf{e}_{2}, \ldots, \mathbf{e}_{d}\right\}$ fixed. The typical element of $L_{01}$ thus has matrix 


$$
\left(\begin{array}{cccccc}
\epsilon_{1} \cosh \alpha & \epsilon_{1} \sinh \alpha & 0 & \ldots & \ldots & 0 \\
\epsilon_{2} \sinh \alpha & \epsilon_{2} \cosh \alpha & 0 & \ldots & \ldots & 0 \\
0 & 0 & 1 & 0 & \ldots & 0 \\
\vdots & \vdots & 0 & 1 & \ddots & \vdots \\
\vdots & \vdots & \vdots & \ddots & \ddots & 0 \\
0 & 0 & 0 & \ldots & 0 & 1
\end{array}\right)
$$

where $\epsilon_{1}$ and $\epsilon_{2}$ are signs. Also, we define $L_{u}^{-}=\left\{U \cdot \Lambda_{\mathbf{v}}: U\right.$ is a space rotation of determinant +1 , and $\Lambda_{\mathbf{v}}$ a velocity boost with $\|\mathbf{v}\|<u$ and $\left.\mathbf{v} \cdot \mathbf{e}_{1}=0\right\}$.

Now, let the graph $G \in \mathscr{I}_{n}$ be given, and let the edges which connect Bands $j$ and $j+1$ of $G$ be $e_{1} \ldots e_{r}$. Let $G^{\prime}=\tau(G)$ and write $e_{i}^{\prime}$ for $\tau\left(e_{i}\right)$. We define $\mathscr{B}_{u} \subset L^{e_{X}(G)}$ and $\mathscr{B}_{u}^{\prime} \subset L^{e_{X}}{ }^{\left(G^{\prime}\right)}$ for each $u<1$, as follows:

$\mathscr{D}_{u}=\left\{\mathbf{g}=\left(g_{e}\right)_{e \in e_{X}(G)}:\right.$ for each $e \notin\left\{e_{1} \ldots e_{r}\right\}, g_{e} \in L_{u} ;$ and for some $\sigma \in L_{01}$ we have $g_{e_{1}} \in L_{u}^{-} \cdot \sigma$ and $\left.g_{e_{2}} \in L_{u} \cdot \sigma(i=2, \ldots . r)\right\}$;

$\mathscr{D}_{u}^{\prime}=\tau\left(\mathscr{C}_{u}\right)=\left\{\left(g_{e}^{\prime}\right)_{e \in e_{X}(G)}:\right.$ for some $\mathbf{g} \in \mathscr{D}_{u}$ we have $g_{\tau(e)}^{\prime}=g_{e}$ for every edge $e \in G\}$.

Note that, because $\mathscr{L}_{u}^{-}$involves a velocity boost with zero component in the $\mathbf{e}_{1}$ direction, the value of $\sigma$ is uniquely determined by $g_{e_{1}}$; and thus, the $\sigma_{i}(i=1, \ldots . r)$ are uniquely determined by $\mathbf{g}$.

Lemma 9.12. The set $\mathscr{L}_{u}$ is an increasing function of $u$, and the union $\cup_{0<u<1} \mathscr{B}_{u}=$ $L^{e_{X}(G)}$.

The proof is obvious.

Now, let us take $\mathbf{g} \in \mathscr{B}_{u}$, and rewrite $g_{e_{1}} \in L_{u}^{-} \cdot L_{01}$ as

$$
\sigma_{1} \cdot \sigma=\sigma_{1} \cdot\left( \pm T^{l} \Lambda_{(\tanh \beta) \mathbf{e}_{1}}\right),
$$

where $\sigma_{1} \in L_{u}^{-}, T$ is time reversal, $l=0$ or 1 , and $\Lambda_{(\tanh \beta) \mathbf{e}_{1}}$ is the velocity boost. Then let us rewrite $g_{e_{i}}(i=2 . . r)$ as $g_{e_{\imath}}=\sigma_{i} \cdot \sigma$ with $\sigma_{i} \in L_{u}(i=2, \ldots . r)$. Likewise for $\mathbf{g}^{\prime} \in \mathscr{B}_{u}^{\prime}$ we write

$$
g_{e_{1}}^{\prime}=\sigma_{1}^{\prime} \cdot \sigma^{\prime}\left(\sigma_{1}^{\prime} \in L_{u}^{-} ; \sigma^{\prime} \in L_{01}\right) ; \sigma^{\prime}= \pm T^{l^{\prime}} \Lambda_{\left(\tanh b e t a^{\prime}\right) \mathbf{e}_{1}} ;
$$

and

$$
g_{e_{\imath}}^{\prime}=\sigma_{i}^{\prime} \cdot \sigma^{\prime} \text { with } \sigma_{i}^{\prime} \in L_{u}(i=2, \ldots . r) .
$$

Note that our sets $\mathscr{B}_{u}\left(\mathscr{B}_{u}^{\prime}\right)$ split naturally into four "components" (from the topological point of view, actually four unions of several components each) with $l\left(l^{\prime}\right)=0$ or 1 and the sign in $(9.12 .1)((9.12 .2))$ positive or negative.

Lemma 9.13. If we restrict Haar measure to $\mathscr{B}_{u}$ it has form

$$
\left(\prod_{e \notin\left\{e_{1} \ldots e_{r}\right\}} d g_{e}\right) \cdot d \nu\left(\sigma_{1}, \ldots, \sigma_{r}\right) \cdot d \beta
$$

on each "component"; where $\nu$ is a finite measure and $d \beta$ is Lebesgue measure on $\mathbb{R}$.

Proof. The set $\mathscr{B}_{u}$ is invariant under the map which applies $\sigma_{0} \in L_{01}$ on the right to each $g_{i}(i=1 . . r)$, and leaves the other $g_{e}$ fixed. Our measure was right invariant Haar measure to begin with, so even when thus restricted it must be of form 


$$
\left(\prod_{e \notin\left\{e_{1} \ldots e_{r}\right\}} d g_{e}\right) \cdot d \nu\left(\sigma_{1}, \ldots, \sigma_{r}\right) \cdot d \gamma
$$

where $d \gamma$ is Haar measure on $L_{01}$. But this is just $d \beta$ on each component, as is well known in the 2-dimensional case because the velocity boosts are a subgroup isomorphic to $(\mathbb{R},+)$ by the map sending $\Lambda_{\tanh \beta \mathbf{e}_{1}}$ to $\beta$. The fact that $\nu$ is finite follows because if $\beta$ is restricted to a bounded set, the associated subset of $\mathscr{B}_{u}$ is precompact in $L^{e_{X}(G)}$ and has finite measure.

Definition 9.14. Let $u \in(0,1)$ be given. With the notation of (9.7-11) we define the reduced second moments

$M_{G, 2}^{u}(\mathbf{f})$ and $M_{G^{\prime}, 2}^{u}\left(\mathbf{f}^{\prime}\right)$, by

$$
M_{G, 2}^{u}(\mathbf{f})=\int_{\mathscr{L}_{u}} \int_{\mathbb{R}(d+1) e(G)} M_{G}(\mathbf{f}, \mathbf{p}, \mathbf{g}) \prod_{\substack{i=1, \ldots . n \\ k=1, \ldots, s_{i}}} \delta\left(\psi_{i, k}(\mathbf{p})\right) d \mathbf{p} d \mathbf{g}
$$

and

$$
M_{G^{\prime}, 2}^{u}\left(\mathbf{f}^{\prime}\right)=\int_{\mathscr{S}_{u}^{\prime}} \int_{\mathbb{R}^{(}(d+1) e(G)} M_{G^{\prime}}\left(\mathbf{f}^{\prime}, \mathbf{p}^{\prime}, \mathbf{g}^{\prime}\right) \prod_{\substack{\imath=1, \ldots . n \\ k=1, \ldots, s_{\imath}}} \delta\left(\psi_{i, k}^{\prime}\left(\mathbf{p}^{\prime}\right)\right) d \mathbf{p}^{\prime} d \mathbf{g}^{\prime},
$$

where $\psi_{i, k}\left(\psi_{i, k}^{\prime}\right)$ are the flows into vertices $X_{i, k}$ of $G\left(G^{\prime}\right)$ as in (3.2.1), and the sets $\mathscr{B}_{u} \subset L^{e_{X}(G)}, \mathscr{D}_{u}^{\prime} \subset L^{e_{X}^{\left(G^{\prime}\right)}}$ are as in (9.12).

Lemma 9.15. The reduced second moments all exist, and for each $f_{1}, \ldots, f_{n}$ in $\mathscr{S}\left(\mathbb{R}^{d+1}\right)$,

$$
\lim _{u \nearrow 1} M_{G, 2}^{u}(\mathbf{f})=M_{G, 2}(\mathbf{f}) \text { and } \lim _{u \nearrow 1} M_{G^{\prime}, 2}^{u}\left(\mathbf{f}^{\prime}\right)=M_{G^{\prime}, 2}\left(\mathbf{f}^{\prime}\right) .
$$

Proof. If the domains $\mathscr{D}_{u}\left(\mathscr{L}_{u}^{\prime}\right)$ are replaced by $L^{e_{X}(G)}\left(L^{e_{X}\left(G^{\prime}\right)}\right)$ in (9.14.1), then we know by Lemma 4.3 that the integrals are convergent to $M_{G, 2}(\mathbf{f}), M_{G^{\prime}, 2}\left(\mathbf{f}^{\prime}\right)$. By Lemma 9.12 and the dominated convergence theorem, we have the result.

Definition 9.16. Returning to (9.10.3) we define

$$
\begin{gathered}
\gamma_{+}\left(\alpha, \mathbf{x}, \boldsymbol{\xi} ; \mathbf{q}, \mathbf{q}^{\prime}\right)=\widehat{\gamma}\left(\alpha,\left(x_{0}, x_{1}\right), \boldsymbol{\xi}, \mathbf{q}^{\prime} \cdot(-\sinh \alpha, \cosh \alpha, 0, \ldots, 0)\right) \times \\
\exp \left(-\mathrm{i}\left(\mathbf{q} \cdot \mathbf{x}+\mathbf{q}^{\prime} \cdot\left(x_{0}, x_{1}, \xi_{2}, \ldots, \xi_{d}\right)\right)\right)
\end{gathered}
$$

so that

$$
\widehat{f}_{j}(\mathbf{q}) \widehat{f}_{j+1}\left(\mathbf{q}^{\prime}\right)=\int_{E^{\prime}} f_{j}(\mathbf{x}) \gamma_{+}\left(\alpha, \mathbf{x}, \boldsymbol{\xi} ; \mathbf{q}, \mathbf{q}^{\prime}\right) d \alpha d \boldsymbol{\xi} d \mathbf{x},
$$

where as in (9.10.3),

$$
E^{\prime}=\left[-\tanh ^{-1} v, \tanh ^{-1} v\right] \times \mathbb{R}^{d-1} \times \operatorname{supp} f_{\jmath} .
$$

Now, let us imitate the process involved in Lemma 4.3, by picking a spanning tree $T_{i}$ for each band of $G$, writing 
$T_{i}^{\prime}=\tau\left(T_{i}\right), \bar{T}=\cup_{i=1}^{n} T_{i}$ and $\bar{T}^{\prime}=\cup_{\imath=1}^{n} T_{i}^{\prime}$; and as in (4.3.2-4) define maps

$$
\begin{gathered}
\epsilon: V_{T}=\mathbb{R}^{(d+1)(e(G) \backslash e(\bar{T}))} \rightarrow \mathbb{R}^{(d+1) e(G)^{\prime}} \\
\eta_{\mathbf{g}}: \mathbb{R}^{(d+1) e(G)} \rightarrow \mathbb{R}^{(d+1) e(G)}
\end{gathered}
$$

and

$$
\zeta_{\mathbf{g}}=\eta_{\mathbf{g}} \circ \epsilon
$$

so that

$$
M_{G, 2}(\mathbf{f})=\int^{\left|e_{X}(G)\right|} \int_{V_{T}} \prod_{\imath=1}^{n}\left(\widehat{f}_{\imath}\left(\phi_{i}(\epsilon(\mathbf{q}))\right)\right) N_{G}\left(\zeta_{\mathbf{g}}(\mathbf{q}), \mathbf{g}\right) d \mathbf{q} d \mathbf{g}
$$

and likewise

$$
M_{G, 2}^{u}(\mathbf{f})=\int_{\mathscr{L}_{u}} \int_{V_{T}} \prod_{\imath=1}^{n}\left(\widehat{f}_{i}\left(\phi_{i}(\epsilon(\mathbf{q}))\right)\right) N_{G}\left(\zeta_{\mathbf{g}}(\mathbf{q}), \mathbf{g}\right) d \mathbf{q} d \mathbf{g} .
$$

Then, let us define the analogous maps for $G^{\prime}$;

$$
\begin{gathered}
\epsilon^{\prime}: V_{T}^{\prime}=\mathbb{R}^{(d+1)\left(e\left(G^{\prime}\right) \backslash e\left(\bar{T}^{\prime}\right)\right)} \rightarrow \mathbb{R}^{(d+1) e\left(G^{\prime}\right)}, \\
\eta_{\mathbf{g}}^{\prime}: \mathbb{R}^{(d+1) e\left(G^{\prime}\right)} \rightarrow \mathbb{R}^{(d+1) e\left(G^{\prime}\right)}
\end{gathered}
$$

and $\zeta_{\mathbf{g}}^{\prime}=\eta_{\mathbf{g}}^{\prime} \circ \epsilon^{\prime}$ so that

$$
M_{G^{\prime}, 2}\left(\mathbf{f}^{\prime}\right)=\int^{\left|e_{X}\left(G^{\prime}\right)\right|} \int_{V_{T}^{\prime}} \prod_{i=1}^{n}\left(\widehat{f}_{i}^{\prime}\left(\phi_{i}^{\prime}\left(\epsilon^{\prime}\left(\mathbf{q}^{\prime}\right)\right)\right)\right) N_{G^{\prime}}\left(\zeta_{\mathbf{g}^{\prime}}^{\prime}\left(\mathbf{q}^{\prime}\right), \mathbf{g}^{\prime}\right) d \mathbf{q}^{\prime} d \mathbf{g}^{\prime}
$$

and likewise

$$
M_{G, 2}^{u}\left(\mathbf{f}^{\prime}\right)=\int^{\mathscr{B}_{u}^{\prime}} \int_{V_{T}^{\prime}} \prod_{i=1}^{n}\left(\widehat{f}_{i}^{\prime}\left(\phi_{i}^{\prime}\left(\epsilon^{\prime}\left(\mathbf{q}^{\prime}\right)\right)\right)\right) N_{G^{\prime}}\left(\zeta_{\mathbf{g}^{\prime}}^{\prime}\left(\mathbf{q}^{\prime}\right), \mathbf{g}^{\prime}\right) d \mathbf{q}^{\prime} d \mathbf{g} .^{\prime}
$$

Definition 9.17. Let then the function $N_{G}^{3}(\mathbf{f}, \mathbf{p}, \mathbf{g})$ be

$$
N_{G}(\mathbf{p}, \mathbf{g}) \cdot \prod_{i=1, \ldots, j-1, j+2, \ldots, n} \widehat{f}_{i}\left(\phi_{i}(\mathbf{p})\right)
$$

and let $N_{G^{\prime}}^{j}\left(\mathbf{f}^{\prime}, \mathbf{p}^{\prime}, \mathbf{g}^{\prime}\right)$ be

$$
\begin{aligned}
& N_{G^{\prime}}\left(\mathbf{p}^{\prime}, \mathbf{g}^{\prime}\right) \cdot \prod_{\imath=1, \ldots, j-1, \jmath+2, \ldots, n} \widehat{f}_{i}^{\prime}\left(\phi_{i}^{\prime}\left(\mathbf{p}^{\prime}\right)\right) \\
= & N_{G^{\prime}}\left(\mathbf{p}^{\prime}, \mathbf{g}^{\prime}\right) \cdot \prod_{i=1, \ldots, j-1, \jmath+2, \ldots, n} \widehat{f}_{i}\left(\phi_{i}^{\prime}\left(\mathbf{p}^{\prime}\right)\right) .
\end{aligned}
$$

These definitions may be substituted in $(9.16 .6,10)$, obtaining 


$$
M_{G, 2}^{u}(\mathbf{f})=\int_{\mathscr{S}_{u}} \int_{V_{T}}\left(\widehat{f}_{\jmath}\left(\phi_{j}(\epsilon(\mathbf{q}))\right) \widehat{f}_{j+1}\left(\phi_{j+1}(\epsilon(\mathbf{q}))\right)\right) N_{G}^{j}\left(\mathbf{f}, \zeta_{\mathbf{g}}(\mathbf{q}), \mathbf{g}\right) d \mathbf{q} d \mathbf{g}
$$

and

$$
\begin{aligned}
& M_{G, 2}^{u}\left(\mathbf{f}^{\prime}\right)=\int^{\mathscr{S}_{u}^{\prime}} \int_{V_{T}^{\prime}}\left(\widehat{f}_{j}^{\prime}\left(\phi_{\jmath}^{\prime}\left(\epsilon^{\prime}\left(\mathbf{q}^{\prime}\right)\right)\right) \widehat{f}_{j+1}^{\prime}\left(\phi_{j+1}^{\prime}\left(\epsilon^{\prime}\left(\mathbf{q}^{\prime}\right)\right)\right)\right) N_{G^{\prime}}^{\jmath}\left(\mathbf{f}^{\prime}, \zeta_{\mathbf{g}^{\prime}}^{\prime}\left(\mathbf{q}^{\prime}\right), \mathbf{g}^{\prime}\right) d \mathbf{q}^{\prime} d \mathbf{g} .^{\prime} \\
& =\int^{\mathscr{S}_{u}^{\prime}} \int_{V_{T}^{\prime}}\left(\widehat{f}_{j+1}\left(\phi_{j}^{\prime}\left(\epsilon^{\prime}\left(\mathbf{q}^{\prime}\right)\right)\right) \widehat{f}_{j}\left(\phi_{j+1}^{\prime}\left(\epsilon^{\prime}\left(\mathbf{q}^{\prime}\right)\right)\right)\right) N_{G^{\prime}}^{\jmath}\left(\mathbf{f}, \zeta_{\mathbf{g}^{\prime}}^{\prime}\left(\mathbf{q}^{\prime}\right), \mathbf{g}^{\prime}\right) d \mathbf{q}^{\prime} d \mathbf{g} .
\end{aligned}
$$

These definitions, though a little cumbersome, enable us to state our key lemma:

Lemma 9.18. For all $u<1$, the integrals obtained by substituting (9.16.2) into (9.17.2) and (9.17.3), namely

$$
\begin{gathered}
M_{G, 2}^{u}(\mathbf{f})=\int_{\mathscr{L}_{u}} \int_{V_{T}} N_{G}^{j}\left(\mathbf{f}, \zeta_{\mathbf{g}}(\mathbf{q}), \mathbf{g}\right) \times \\
{\left[\int_{E^{\prime}} f_{j}(\mathbf{x}) \gamma_{+}\left(\alpha, \mathbf{x}, \boldsymbol{\xi} ; \phi_{j}(\epsilon(\mathbf{q})), \phi_{j+1}(\epsilon(\mathbf{q})) d \alpha d \boldsymbol{\xi} d \mathbf{x}\right] d \mathbf{q} d \mathbf{g}\right.}
\end{gathered}
$$

and

$$
\begin{gathered}
M_{G^{\prime}, 2}^{u}\left(\mathbf{f}^{\prime}\right)=\int_{\mathscr{S}_{u}^{\prime}} \int_{V_{T}^{\prime}} N_{G^{\prime}}^{j}\left(\mathbf{f}^{\prime}, \zeta_{\mathbf{g}^{\prime}}^{\prime}\left(\mathbf{q}^{\prime}\right), \mathbf{g}^{\prime}\right) \times \\
{\left[\int_{E^{\prime}} f_{j}^{\prime}(\mathbf{x}) \gamma_{+}\left(\alpha, \mathbf{x}, \boldsymbol{\xi} ; \phi_{j}^{\prime}\left(\epsilon^{\prime}\left(\mathbf{q}^{\prime}\right)\right), \phi_{j+1}^{\prime}\left(\epsilon^{\prime}\left(\mathbf{q}^{\prime}\right)\right) d \alpha d \boldsymbol{\xi} d \mathbf{x}\right] d \mathbf{q}^{\prime} d \mathbf{g}^{\prime}\right.}
\end{gathered}
$$

in fact converge as multiple integrals on $\mathscr{D}_{u} \times V_{T} \times E^{\prime}$ and $\mathscr{L}_{u}^{\prime} \times V_{T}^{\prime} \times E^{\prime}$ respectively (here $E^{\prime}$ is as in (9.10.3)). Furthermore, they are the same.

Proof. We consider the set $E_{3} \subset\left(\mathbb{R}^{d+1} \times L\right)^{r}$ consisting of sequences $\left(\mathbf{q}_{e_{i}}, g_{e_{i}}\right)_{i=1}^{r} \in$ $\mathbb{R}^{d+1} \times L$ such that (as in (9.12.1-2)) we have $g_{e_{2}}=\sigma_{i} \cdot \sigma$ (9.18.3) with $\sigma_{1} \in$ $L_{u}^{-}, \sigma \in L_{01}, \sigma_{i} \in L_{u}(i=2, \ldots . r)$. Then let $E_{K, \alpha} \subset E_{3}$ denote the subset of sequences such that, additionally, $\left\|g_{e_{i}} \mathbf{q}_{e_{i}}\right\| \leq K(i=1, \ldots . r)$ (9.18.4) and (for the given value $\left.\alpha \in\left[-\tanh ^{-1} v, \tanh ^{-1} v\right]\right)$ we have

$$
\left|\left(\sum_{\imath=1}^{r} \mathbf{q}_{e_{\imath}}\right) \cdot(-\sinh \alpha, \cosh \alpha, 0, \ldots, 0)\right| \leq K \text {. }
$$

Let us say $\sigma= \pm T^{l} \cdot \Lambda_{(\tanh \beta) \mathbf{e}_{1}}$ where $T$ is time reversal, $\beta \in \mathbb{R}$. Then, the elements $\pm \sigma_{i} T^{l}(i=1 \ldots r)$ are drawn from a compact set of invertible linear maps so there is a $K_{1}$ (depending on $u$ ) such that if $\left\|g_{e_{i}} \mathbf{q}_{e_{i}}\right\| \leq K$ then $\left\|\Lambda_{(\tanh \beta) \mathbf{e}_{1}} \mathbf{q}_{e_{i}}\right\| \leq K_{1} \cdot K$. (9.18.6).

Write

$$
\mathbf{q}_{e_{\imath}}=\left(\mu_{i} \cosh \beta-\lambda_{i} \sinh \beta, \mu_{\imath} \sinh \beta+\lambda_{\imath} \cosh \beta, q_{i, 2}, q_{i, 3}, \ldots, q_{i, d}\right)
$$

and after applying the transformation $\Lambda_{(\tanh \beta) \mathbf{e}_{1}}$ we have vectors

$$
\Lambda_{(\tanh \beta) \mathbf{e}_{1}} \mathbf{q}_{\mathbf{e}_{\imath}}=\left(\mu_{i} \cosh 2 \beta, \mu_{i} \sinh 2 \beta+\lambda_{i}, q_{\imath, 2}, q_{i, 3}, \ldots, q_{i, d}\right)
$$


$(i=1, \ldots . r)$. Hence, $\left|\mu_{i}\right| \leq K K_{1} \operatorname{sech} 2 \beta,\left\|\left(q_{i, 2}, q_{i, 3}, \ldots, q_{i, d}\right)\right\| \leq K K_{1}$ and $\mid \lambda_{i}+$ $\mu_{\imath} \sinh 2 \beta \mid \leq K K_{1}$ so $\left|\lambda_{i}\right| \leq 2 K K_{1}$ (9.18.8). Also

$$
\begin{gathered}
\left|\left(\sum_{i=1}^{r} \mathbf{q}_{e_{i}}\right) \cdot(-\sinh \alpha, \cosh \alpha, 0, \ldots, 0)\right| \\
=\left|\left(\sum_{i=1}^{r} \lambda_{i}\right) \cosh (\alpha+\beta)+\left(\sum_{i=1}^{r} \mu_{i}\right) \sinh (\beta-\alpha)\right| \leq K_{1},
\end{gathered}
$$

therefore

$$
\left|\sum_{i=1}^{r} \lambda_{i}\right| \leq K_{1} \operatorname{sech}(\alpha+\beta)+r K K_{1} \operatorname{sech}(2 \beta) \sinh (\beta-\alpha) .
$$

But $\alpha$ comes from a bounded set so for a suitable $K_{2}=K_{2}(v)$,

$$
\left|\sum_{i=1}^{r} \lambda_{i}\right| \leq\left(K_{1} K_{2}+r K K_{1} K_{2}\right) \operatorname{sech}(\beta)
$$

The Jacobian involved in the transformation sending $\mathbf{q}_{e_{i}}$ to $\left(\lambda_{\imath}, \mu_{\imath}, q_{\imath, 2}, q_{\imath, 3}, \ldots, q_{\imath, d}\right)$ $(i=1 . . r)$ is $(\cosh 2 \beta)^{r}$. So for fixed $g_{e_{1}} \ldots g_{e_{r}}$ the volume of the cross section of $E_{K, \alpha}$ as the $\mathbf{q}_{e_{i}}$ vary is at most

$(\cosh 2 \beta)^{r} \cdot\left(2 K K_{1} \operatorname{sech} 2 \beta\right)^{r} \cdot \operatorname{vol}\left\{\left(\lambda_{1}, \ldots, \lambda_{r}\right) \in\left[-2 K K_{1}, 2 K K_{1}\right]^{r}:\right.$

$$
\left.\left|\lambda_{1}+\ldots+\lambda_{r}\right| \leq K_{2} K_{1}(1+r K) \operatorname{sech} \beta\right\} \cdot\left(\operatorname{vol} B_{d-1}(0, K)\right)^{r},
$$

(where $\operatorname{vol} B_{d-1}(0, K)$ is the volume of a ball of radius $K$ in dimension $d-1$, which is the domain in which the vectors $\left(q_{i, 2}, q_{i, 3}, \ldots, q_{i, d}\right)$ are allowed to vary)

$$
\begin{gathered}
\leq\left(2 K K_{1}\right)^{r}\left(4 K K_{1}\right)^{r-1} \cdot K_{2} K_{1}(1+r K) \operatorname{sech} \beta \cdot\left(\operatorname{vol} B_{d-1}(0, K)\right)^{r} \\
\leq K^{r(d+1)} K_{3} \operatorname{sech} \beta
\end{gathered}
$$

shall we say, where $K_{3}$ depends on $r, v$ and $u$. Now the element of Haar measure on the elements $g_{e_{1}} \ldots g_{e_{r}}$ is $d \nu\left(\sigma_{1} \ldots \sigma_{r}\right) \cdot d \beta$ where $d \nu$ is finite (let's say $|d \nu|=K_{4}$ ); therefore by $(9.18 .10)$ we have

$$
\text { vol } E_{K, \alpha} \leq \int_{-\infty}^{\infty} K^{r(d+1)} K_{3} K_{4} \operatorname{sech}(\beta) d \beta \leq 4 K_{3} K_{4} K^{r(d+1)}
$$

Now, the factor $\gamma_{+}\left(\alpha, \mathbf{x}, \boldsymbol{\xi} ; \phi_{j}(\epsilon(\mathbf{q}))\right.$, in our integrand (9.18.1), is

$$
o\left((1+\|\boldsymbol{\xi}\|)^{-R} \cdot\left(1+\left|\phi_{j+1}(\epsilon(\mathbf{q})) \cdot(-\sinh \alpha, \cosh \alpha, 0, \ldots, 0)\right|\right)^{-R}\right)
$$

for any given $R>0$ (see (9.8.1), (9.8.2), (9.16.1)). Now if $e$ is an edge of $e(G) \backslash \bar{T}$ there is a factor $\hat{\theta}\left(g_{e} \mathbf{q}_{e}\right)$ in $N_{G}^{j}$ which is $o\left(\left(1+\left\|\mathbf{q}_{e}\right\|\right)^{-R}\right)$ for any given $R>0$ because $g_{e}$ is only allowed to vary within a compact set for such $e$. For $e=e_{1}, e_{2}, \ldots, e_{r}$ we can at least say that the integrand has a factor which is $o\left(\left(1+\left\|g_{e} \mathbf{q}_{e}\right\|\right)^{-R}\right)$ for any $\mathrm{R}$. Hence, for each $R>0$ there is a $C=C(R)$ such that $(9.18 .1)$ is at most 


$$
\begin{gathered}
C(R) \int_{E^{\prime} \times V_{T} \times \mathscr{L}_{u}}(1+\|\boldsymbol{\xi}\|)^{-R} \prod_{e \in e(G) \backslash \bar{T} \backslash\left\{e_{1}, \ldots, e_{r}\right\}}\left(1+\left\|\mathbf{q}_{e}\right\|\right)^{-R} \times \\
\left(1+\left|\phi_{j+1}(\epsilon(\mathbf{q})) \cdot(-\sinh \alpha, \cosh \alpha, 0, \ldots, 0)\right|\right)^{-R} \cdot \prod_{i=1}^{r}\left(1+\left\|g_{e_{i}} \mathbf{q}_{e_{i}}\right\|\right)^{-R} d \alpha d \boldsymbol{\xi} d \mathbf{x} d \mathbf{q} d \mathbf{g} .
\end{gathered}
$$

But $\phi_{\jmath+1}\left(\epsilon(\mathbf{q})\right.$ is of form $\sum_{i=1}^{r} \mathbf{q}_{e_{i}}+\sum_{(\text {some other edges })} \pm \mathbf{q}_{e}$. Since the other $\mathbf{q}_{e}$ already appear in factors $\left(1+\left\|\mathbf{q}_{e}\right\|\right)^{-R}$ in the integrand (9.18.12), we infer there is a $C^{\prime}(R)$ such that the integral is at most

$$
\begin{gathered}
C^{\prime}(R) \int_{E^{\prime} \times V_{T} \times \mathscr{G}_{u}}(1+\|\boldsymbol{\xi}\|)^{-R} \prod_{e \in e(G) \backslash \bar{T} \backslash\left\{e_{1}, \ldots, e_{r}\right\}}\left(1+\left\|\mathbf{q}_{e}\right\|\right)^{-R} \times \\
\left(1+\left|\left(\sum_{i=1}^{r} \mathbf{q}_{e_{i}}\right) \cdot(-\sinh \alpha, \cosh \alpha, 0, \ldots, 0)\right|\right)^{-R} \cdot \prod_{\imath=1}^{r}\left(1+\left\|g_{e_{i}} \mathbf{q}_{e_{\imath}}\right\|\right)^{-R} d \alpha d \boldsymbol{\xi} d \mathbf{x} d \mathbf{q} d \mathbf{g} .
\end{gathered}
$$

Fix $K>0$, and let us integrate with respect to the $g_{e_{\imath}}$ and $\mathbf{q}_{e_{i}}(i=1, \ldots . r)$ over the region where

$$
\max \left\{\left\|g_{e_{i}} \mathbf{q}_{e_{\imath}}\right\|(i=1, \ldots . r),\left|\left(\sum_{i=1}^{r} \mathbf{q}_{e_{i}}\right) \cdot(-\sinh \alpha, \cosh \alpha, 0, \ldots, 0)\right|\right\} \in(K-1, K] .
$$

By our estimate $(9.18 .11)$ on the volume available, we get at most

$$
C^{\prime}(R)\left(1+\|\boldsymbol{\xi}\|^{-R} \prod_{e \in e(G) \backslash \bar{T} \backslash\left\{e_{1}, \ldots, e_{r}\right\}}\left(1+\left\|\mathbf{q}_{e}\right\|\right)^{-R}(1+(K-1))^{-R} \cdot 4 K_{3} K_{4} K^{r(d+1)} .\right.
$$

If $R>r(d+1)+1$ then summing from $K=1$ to infinity we have a finite answer, at most

$$
C_{4} \cdot(1+\|\boldsymbol{\xi}\|)^{-R} \prod_{e \in e(G) \backslash \bar{T} \backslash\left\{e_{1}, \ldots, e_{r}\right\}}\left(1+\left\|\mathbf{q}_{e}\right\|\right)^{-R},
$$

where $C_{4}=C_{4}(R, v, u)$ is a constant. So (9.18.1) is at most

$$
\begin{gathered}
C_{4} \cdot \int_{E_{4}}(1+\|\boldsymbol{\xi}\|)^{-R}\left(\prod_{e \in e(G) \backslash \bar{T} \backslash\left\{e_{1}, \ldots, e_{r}\right\}}\left(1+\left\|\mathbf{q}_{e}\right\|\right)^{-R} d \mathbf{q}_{e}\right) \times \\
\left(\prod_{e \in e_{X}(G) \backslash\left\{e_{1} \ldots e_{r}\right\}} d g_{e}\right) d \boldsymbol{\xi} d \alpha d \mathbf{x},
\end{gathered}
$$

where

$$
E_{4}=\mathbb{R}^{(d+1)\left(e(G) \backslash \bar{T} \backslash\left\{e_{1} \ldots e_{r}\right\}\right)} \times L_{u}^{e_{X}(G) \backslash\left\{e_{1} \ldots e_{r}\right\}} \times E^{\prime} .
$$

Now it is only $\boldsymbol{\xi}$ and the remaining $\mathbf{q}_{e}$ that are integrated over a noncompact region; the integral is finite for $R>d+2$, and uniformly bounded in the other variables, so we know that the multiple integral (9.18.1) converges absolutely, for each fixed $u<1$. In the same way, the multiple integral (9.18.2) converges absolutely, and the "large square brackets" of (9.18.1) and (9.18.2) may mentally be removed. 
We now prove that the multiple integral $(9.18 .2)$ is equal to $(9.18 .1)$, by an appropriate substitution. In fact, we fix an $\alpha \in\left[-\tanh ^{-1} v, \tanh ^{-1} v\right]$, and we make the following substitutions among the other variables.

Definition 9.19. Given a fixed $\alpha$, we make the substitutions

$$
\mathbf{q}_{e}^{\prime}= \begin{cases}\mathbf{q}_{f}, & \text { if } e=\tau(f), f \notin\left\{e_{1} \ldots e_{r}\right\} \\ -T_{\alpha} \mathbf{q}_{f}, & \text { if } e=\tau(f), f=e_{i} \text { for some } i=1, \ldots . r\end{cases}
$$

and

$$
g_{e}^{\prime}=\left\{\begin{array}{l}
g_{f}, \text { if } e=\tau(f), f \notin\left\{e_{1} \ldots e_{r}\right\} \\
g_{f} \circ T_{\alpha}, \text { if } e=\tau(f), f=e_{i} \text { for some } i=1, \ldots .
\end{array}\right.
$$

where $T_{\alpha}$ is time reversal in a frame of reference with time direction $(\cosh \alpha, \sinh \alpha, 0, \ldots, 0)$.

Note that since $T_{\alpha} \in L_{01}$, this substitution sends the one domain of integration onto the other; and our measure is invariant under it (9.13).

Lemma 9.20. With these values of $\mathbf{g}^{\prime}, \mathbf{q}^{\prime}$, we have

$$
N_{G^{\prime}}^{j}\left(\mathbf{f}^{\prime}, \zeta_{\mathbf{g}^{\prime}}^{\prime}\left(\mathbf{q}^{\prime}\right), \mathbf{g}^{\prime}\right)=N_{G}^{\jmath}\left(\mathbf{f}, \zeta_{\mathbf{g}}(\mathbf{q}), \mathbf{g}\right) .
$$

Furthermore,

$$
\left(\phi_{\jmath+1}(\mathbf{p})-\phi_{j}^{\prime}\left(\mathbf{p}^{\prime}\right)\right) \cdot(-\sinh \alpha, \cosh \alpha, 0 \ldots 0)=0
$$

and

$$
\phi_{j}(\mathbf{p}) \cdot \mathbf{x}+\phi_{j+1}(\mathbf{p}) \cdot\left(x_{0}, x_{1}, \xi_{2}, \ldots, \xi_{d}\right)=\phi_{j+1}^{\prime}\left(\mathbf{p}^{\prime}\right) \cdot \mathbf{x}+\phi_{j}^{\prime}\left(\mathbf{p}^{\prime}\right) \cdot\left(x_{0}, x_{1}, \xi_{2}, \ldots, \xi_{d}\right) .
$$

Proof. Now the orientation of the edge $e=(u, v)$ (i.e., the question of which of the two vertices is "above" the other) is reversed by the map $e \rightarrow e^{\prime}=\tau(e)$ if and only if $e \in\left\{e_{1}, \ldots, e_{r}\right\}$.

Write then $\zeta=\zeta_{\mathbf{g}}(\mathbf{q})$ and $\boldsymbol{\zeta}^{\prime}=\zeta_{\mathbf{g}^{\prime}}^{\prime}\left(\mathbf{q}^{\prime}\right)$. We define a vector $\tau(\boldsymbol{\zeta}) \in \mathbb{R}^{(d+1) e\left(G^{\prime}\right)}$ rather as in (9.19), by

$$
\tau(\boldsymbol{\zeta})_{e}= \begin{cases}\zeta_{f}, & \text { if } e \notin\left\{e_{1}, \ldots, e_{r}\right\}, \text { where } e=\tau(f) \\ -T_{\alpha} \zeta_{f}, & \text { if } e \in\left\{e_{1}, \ldots, e_{r}\right\}, \text { where } e=\tau(f) .\end{cases}
$$

We claim that $\tau(\zeta)=\zeta^{\prime}$. Considering the matter edge by edge, and using (4.3.2-4) we see that $\tau(\zeta)_{e}$ is obviously equal to $\zeta_{e}^{\prime}$ if $e \notin \bar{T}^{\prime}$ because both are equal to $\mathbf{q}_{e^{\prime}}^{\prime}$. Now the whole point about (4.3.2-4) is that $\zeta_{\mathbf{g}}(\mathbf{q})$ will be the unique vector extending $\mathbf{q}$ from $\mathbb{R}^{(d+1)(e(G) \backslash \bar{T})}$ to $\mathbb{R}^{(d+1) e(G)}$ in such a way that $\psi_{i, j}\left(G, \zeta_{\mathbf{g}}(\mathbf{q}), \mathbf{g}\right)=0$ for all $i, j$. But then, the vector $\tau(\boldsymbol{\zeta})$ will extend $\mathbf{q}^{\prime}$ from $\mathbb{R}^{(d+1) e\left(G^{\prime}\right) \backslash \bar{T}^{\prime}}$ to $\mathbb{R}^{(d+1) e\left(G^{\prime}\right)}$ in such a way that $\psi_{i, j}\left(G^{\prime}, \tau(\boldsymbol{\zeta}), \mathbf{g}^{\prime}\right)=0$ for all $i$ and $j$; for the functions $\psi_{\imath, j}$ count products $g_{e} \zeta_{e}$ positively or negatively according to their orientation (3.2); and in view of (9.19), (9.20.4) we have negated our products (that is, obtained $\left.g_{e} \zeta_{e}=-g_{e^{\prime}}^{\prime}(\tau(\zeta))_{e^{\prime}}\right)$ precisely in the $r$ cases when the orientation changes. Therefore, $\tau(\boldsymbol{\zeta})=\boldsymbol{\zeta}^{\prime}$.

Let's now establish (9.20.1). The functions $N_{G^{\prime}}^{j}\left(\mathbf{f}^{\prime}, \zeta_{\mathbf{g}^{\prime}}^{\prime}\left(\mathbf{q}^{\prime}\right), \mathbf{g}^{\prime}\right)$ and $N_{G}^{j}\left(\mathbf{f}, \zeta_{\mathbf{g}}(\mathbf{q}), \mathbf{g}\right)$ depend on values $\widehat{f}_{i}$ for $i$ not equal to the special values $j$ and $j+1(9.17 .1)$, which are 
certainly unchanged when we replace $G$ with $G^{\prime}$ and $\zeta$ with $\boldsymbol{\zeta}^{\prime}$, because for crossing edges other than the special ones $\left\{e_{1}, \ldots, e_{r}\right\}$, we just have $\zeta_{e}=\mathbf{q}_{e}=\mathbf{q}_{e^{\prime}}^{\prime}=\zeta_{e^{\prime}}^{\prime}$. The functions $N_{G}^{j}\left(\mathbf{f}, \zeta_{\mathbf{g}}(\mathbf{q}), \mathbf{g}\right)$ and $N_{G^{\prime}}^{j}\left(\mathbf{f}^{\prime}, \zeta_{\mathbf{g}^{\prime}}^{\prime}\left(\mathbf{q}^{\prime}\right), \mathbf{g}^{\prime}\right)$ are apart from that dependent only on $N_{G}\left(\zeta_{\mathbf{g}}(\mathbf{q}), \mathbf{g}\right)$ and $N_{G^{\prime}}\left(\zeta_{\mathbf{g}^{\prime}}^{\prime}\left(\mathbf{q}^{\prime}\right), \mathbf{g}^{\prime}\right)$. These are, by (4.1.2), products of the functions $N_{G}^{e}$ (respectively, $N_{G^{\prime}}^{e^{\prime}}$ ), which we claim are equal if, as usual, we have $e^{\prime}=\tau(e)$.

Proof of Claim. For (4.1.3-6) show us that the $N_{G}^{e}$ have only the following dependencies.

They are zero unless all the $\zeta_{e}$ are timelike. They depend on the quantities $\mathrm{H}\left(L_{e}\right)$, where $L_{e}$ is the time coordinate of $\zeta_{e}$. Looking at (9.20.4) we see that - since $T_{\alpha}$ involves a time reversal - these quantities are unchanged when we replace $e$ by $\tau(e)$ and $\zeta$ by $\zeta^{\prime}$ because we have at most changed $\zeta_{e}$ by acting with an element of $L \uparrow$, namely $-T_{\alpha}$.

They depend on quantities $\mu\left(\zeta_{e}^{2}\right)$ which are unchanged when we make the substitution for the same reason.

Lastly, they depend on factors $\left|\widehat{\theta}\left(g_{e} \zeta_{e}\right)\right|^{2}$. By (9.19) and (9.20.4), $g_{e} \zeta_{e}$ has at most a change of sign when we make the substitution; but of course, $\theta$ is a real valued function so $|\widehat{\theta}|$ is even. Thus (9.20.1) is proved. In order to prove (9.20.2) we point out that writing $\mathbf{p}=\zeta_{\mathbf{g}}(\mathbf{q}), \mathbf{p}^{\prime}=\zeta_{\mathbf{g}^{\prime}}^{\prime}\left(\mathbf{q}^{\prime}\right)$, we have

$$
\phi_{j}(\mathbf{p})+\phi_{j+1}(\mathbf{p})=\phi_{j}^{\prime}\left(\mathbf{p}^{\prime}\right)+\phi_{j+1}^{\prime}\left(\mathbf{p}^{\prime}\right)
$$

Also,

$$
=\left(\text { total flow out of bands } j, j+1 \text { of } G\left(\text { or } G^{\prime}\right)\right) \text {. }
$$

$\phi_{j}^{\prime}=\left(\right.$ flow out of Band $\mathrm{j}$ of $G^{\prime}$ from other than Band $\left.j+1\right)+\sum_{i=1}^{r} \mathbf{q}_{e_{\imath}^{\prime}}^{\prime}$

$=($ flow out of Band $\mathrm{j}+1$ of $G$ from other than Band $j)+\sum_{i=1}^{r} \mathbf{q}_{e_{\imath}^{\prime}}^{\prime}$

$$
=\phi_{j+1}+\sum_{i=1}^{r}\left(\mathbf{q}_{e_{\imath}^{\prime}}^{\prime}+\mathbf{q}_{e_{i}}\right)=\phi_{j+1}+\sum_{i=1}^{r}\left(-T_{\alpha} \mathbf{q}_{e_{i}}+\mathbf{q}_{e_{i}}\right) .
$$

Hence, $\phi_{j}^{\prime}\left(\mathbf{p}^{\prime}\right)-\phi_{j+1}(\mathbf{p})$ is in direction $(\cosh \alpha, \sinh \alpha, 0,0, . .0)$. Likewise, $\phi_{j+1}^{\prime}\left(\mathbf{p}^{\prime}\right)-$ $\phi_{j}(\mathbf{p})$ is in direction $(\cosh \alpha, \sinh \alpha, 0,0, . .0)$. This proves both of the last assertions because we obtain the last line $(9.20 .3)$ from $\left(\phi_{j}+\phi_{j+1}\right) \cdot \mathbf{x}=\left(\phi_{j}^{\prime}+\phi_{j+1}^{\prime}\right) \cdot \mathbf{x}$ and $\left(\phi_{\jmath+1}-\phi_{\jmath}^{\prime}\right) \cdot\left(0,0, \xi_{2}-x_{2}, \xi_{3}-x_{3}, \ldots, \xi_{d}-x_{d}\right)=0$.

Corollary 9.21. If we substitute for $\mathbf{q}^{\prime}, \mathbf{g}^{\prime}$ as above, we find that for each fixed $\alpha \in$ $\left[-\tanh ^{-1} v, \tanh ^{-1} v\right]$ the integral

$$
\begin{aligned}
& \int_{E_{5}} N_{G}^{j}\left(\mathbf{f}, \zeta_{\mathbf{g}}(\mathbf{q}), \mathbf{g}\right) \cdot f_{j}(\mathbf{x}) \cdot \gamma_{+}\left(\alpha, \mathbf{x}, \boldsymbol{\xi}, \phi_{j}(\epsilon(\mathbf{q})), \phi_{j+1}(\epsilon(\mathbf{q}))\right) d \mathbf{x} d \boldsymbol{\xi} d \mathbf{q} d \mathbf{g} \\
= & \int_{E_{6}} N_{G^{\prime}}^{j}\left(\mathbf{f}^{\prime}, \zeta_{\mathbf{g}^{\prime}}^{\prime}\left(\mathbf{q}^{\prime}\right), \mathbf{g}^{\prime}\right) \cdot f_{j}(\mathbf{x}) \cdot \gamma_{+}\left(\alpha, \mathbf{x}, \boldsymbol{\xi}, \phi_{j+1}^{\prime}\left(\epsilon^{\prime}\left(\mathbf{q}^{\prime}\right)\right), \phi_{j}^{\prime}\left(\epsilon^{\prime}\left(\mathbf{q}^{\prime}\right)\right)\right) d \mathbf{x} d \boldsymbol{\xi} d \mathbf{q} d \mathbf{g},
\end{aligned}
$$

where

$$
E_{5}=\operatorname{supp} f_{j} \times \mathbb{R}^{d-1} \times V_{T} \times \mathscr{B}_{u}
$$

and

$$
E_{6}=\operatorname{supp} f_{j} \times \mathbb{R}^{d-1} \times V_{T}^{\prime} \times \mathscr{D}_{u}^{\prime}
$$


Consequently, when we perform the final integration with respect to $\alpha$ and obtain (9.18.1,2), we find that $M_{G, 2}^{u}(\mathbf{f})=M_{G^{\prime}, 2}^{u}\left(\mathbf{f}^{\prime}\right)$.

Proof. The reader may verify that if one integrates (9.21.1) with respect to $\alpha$, one obtains (9.18.1) on the left and (9.18.2) on the right. By (9.16.1) the function $\gamma_{+}\left(\alpha, \mathbf{x}, \boldsymbol{\xi}, \phi_{1}, \phi_{2}\right)$ has a dependence on $\phi_{1}$ and $\phi_{2}$ only in terms of $\phi_{2}$. $(-\sinh \alpha, \cosh \alpha, 0 . .0)$ and $\phi_{1} \cdot \mathbf{x}+\phi_{2} \cdot\left(x_{0}, x_{1}, \xi_{2}, \ldots, \xi_{d}\right)$. (9.20.2,3) are therefore exactly what we need to declare the two versions in (9.21.1) equal. The $N_{G}$ functions are equal by (9.20.1), and the result follows. Thus we conclude the proof of (9.21), hence of (9.18).

Corollary 9.22. When the functions $f_{j}$ and $f_{j+1}$ are spacelike separated, the Wightman functions $W_{n}\left(f_{1}, \ldots, f_{n}\right)$ and $W_{n}\left(f_{1}, \ldots, f_{j-1}, f_{j+1}, f_{j}, f_{j+2}, f_{j+3}, \ldots, f_{n}\right)$ are indeed equal.

The proof is now obvious; take the limit as $u \nearrow 1$ of the identity $M_{G, 2}^{u}(\mathbf{f})=$ $M_{G^{\prime}, 2}^{u}\left(\mathbf{f}^{\prime}\right)$, and we have $M_{G, 2}(\mathbf{f})=M_{G^{\prime}, 2}\left(\mathbf{f}^{\prime}\right)$, which establishes (9.7). Multiply this identity by the constant given in (3.5), and sum over all $G \in \mathscr{G}_{n}$ as in (3.6), and we have the result for the case when supports of $f_{j}$ and $f_{j+1}$ are spacelike separated in the $\mathbf{e}_{1}$ direction. This is, by Lemma 9.4, enough to establish the general case. Thus we bring $\S 9$ to a close.

\section{First Steps with the New Theories}

Well, we have arrived; the functions $W_{n}\left(f_{1}, \ldots, f_{n}\right)$ defined back in $\S 3$ are indeed the Wightman functions of a Hermitian scalar field theory. We have found a fairly natural and explicit Hilbert space of quantum theory states $\left(\mathscr{H} \mathscr{C}^{0} \subset \mathscr{H} \mathscr{C}\right.$ as in $\S \S 5$ and 8); and we have found that there is a mass gap $m_{0}$, where $m_{0}$ is the lower end of the support of the smeared "mass density function" defined back in (1.1). At this point we were going to make optimistic comments about possible nontrivial scattering amplitudes; but in view of the "Rehren decomposition," [Reh1], we certainly should not do so. We are, however, cautiously optimistic that some of the ideas in the construction may prove useful. I think the main problem with this construction is that Locality was added last, and in doing so, we gave the theory one too many symmetries - too much "smoothness" - and paved the way for the Rehren decomposition. I think we ought to try something broadly similar starting from a family of linear maps that was transparently local to begin with.

\section{References}

[GJ] Glimm, J., Jaffe, A: Quantum Physics. 2nd ed. Berlin, Heidelberg, New York: Springer, 1987

[GJ1] Glimm, J., Jaffe, A: A $\lambda\left(\phi^{4}\right)_{2}$ quantum field theory without cutoffs I. Phys. Rev. 176, 1945-1951 (1968)

[GJ2] Glimm, J., Jaffe, A: A $\lambda\left(\phi^{4}\right)_{2}$ quantum field theory without cutoffs II. The field operators and the approximate vacuum. Ann. Math 91, 362-401 (1970)

[GJ3] Glimm, J., Jaffe, A: A $\lambda\left(\phi^{4}\right)_{2}$ quantum field theory without cutoffs III. The physical vacuum. Acta. Math 125, 204-267 (1970)

[GJ4] Glimm, J., Jaffe, A: A $\lambda\left(\phi^{4}\right)_{2}$ quantum field theory without cutoffs IV. Perturbations of the Hamiltonian. J.Math. Phys. 13, 1568-1584 (1972)

[GJ5] Glimm, J., Jaffe, A: Positivity of the $\left(\phi^{4}\right)_{3}$ Hamiltonian. Fortschr. Phys. 21, 327-376 (1973) 
[GJ6] Glimm, J., Jaffe, A: A remark on the existence of $\left(\phi^{4}\right)_{4}$. Phys. Rev. Lett. 33, 440-442 (1974)

[H] Haag, R: Local Quantum Physics. Berlin-Heidelberg-New York: Springer, 1993

[I] Iagolnitzer, I.: Scattering in Quantum Field theories. Princeton series in Physics, 1992.

[IZ] Itzykson, C., Zuber, J.: Quantum Field Theory. New York: McGraw-Hill, 1980

[Reh1] K.H.Rehren: Comments on a recent solution to Wightman's axioms. To appear

[SW] Streater, R.F., Wightman, A.S.: PCT, Spin and Statistics, and all that. New York: Benjamin, 1964

Communicated by D. Brydges

This article was processed by the author

using the Springer-Verlag TEX PJourlg macro package 1991. 
